\title{
A graphic approach to gauge invariance induced identity
}

\author{
Linghui Hou ${ }^{a, 1}$ and Yi-Jian $\mathbf{D u} u^{a, b, 2}$ \\ ${ }^{a}$ Center for Theoretical Physics, School of Physics and Technology, Wuhan University, \\ No. 299 Bayi Road, Wuhan 430072, China \\ ${ }^{b}$ Suzhou Institute of Wuhan University, \\ No. 377 Linquan Street, Suzhou, 215123, China \\ E-mail: hlh@whu.edu.cn, yijian.du@whu.edu.cn
}

ABSTRACT: All tree-level amplitudes in Einstein-Yang-Mills (EYM) theory and gravity (GR) can be expanded in terms of color ordered Yang-Mills (YM) ones whose coefficients are polynomial functions of Lorentz inner products and are constructed by a graphic rule. Once the gauge invariance condition of any graviton is imposed, the expansion of a tree level EYM or gravity amplitude induces a nontrivial identity between color ordered YM amplitudes. Being different from traditional Kleiss-Kuijf (KK) and Bern-Carrasco-Johansson (BCJ) relations, the gauge invariance induced identity involves polarizations in the coefficients. In this paper, we investigate the relationship between the gauge invariance induced identity and traditional BCJ relations. By proposing a refined graphic rule, we prove that all the gauge invariance induced identities for single trace tree-level EYM amplitudes can be precisely expanded in terms of traditional BCJ relations, without referring any property of polarizations. When further considering the transversality of polarizations and momentum conservation, we prove that the gauge invariance induced identity for tree-level GR (or pure YM) amplitudes can also be expanded in terms of traditional BCJ relations for YM (or bi-scalar) amplitudes. As a byproduct, a graph-based BCJ relation is proposed and proved.

KEywords: Scattering Amplitudes, Gauge Symmetry

ARXIV EPRINT: 1811.12653

\footnotetext{
${ }^{1}$ The unusual ordering of authors is just to let authors get proper recognition of contributions under outdated practice in China.

${ }^{2}$ Corresponding author
} 


\section{Contents}

1 Introduction 1

2 Expansion of tree level single-trace EYM amplitudes, gauge invariance induced identity and traditional BCJ relation 3

2.1 Recursive expansion of tree level single-trace EYM amplitudes 3

2.2 Graphic rule for the pure YM expansion of tree level single-trace EYM

amplitudes 4

2.3 Gauge invariance induced identity from tree level single-trace EYM amplitudes 5

2.4 BCJ relation 6

3 Refined graphic rule and the main idea $\quad 6$

$\begin{array}{lll}3.1 & \text { Refined graphic rule for single trace tree-level EYM amplitudes } & 6\end{array}$

3.1.1 Examples for the refined graphic rule $\quad 8$

$\begin{array}{ll}3.2 & \text { The main idea }\end{array}$

4 Direct evaluations $\quad 10$

$\begin{array}{lll}4.1 \text { A typical example } & 10\end{array}$

$\begin{array}{lll}4.2 & \text { Common features of the examples } & 14\end{array}$

5 General structure of skeletons $\quad 16$

6 Constructing all physical and spurious graphs for a given skeleton $\quad 19$

$\begin{array}{ll}\text { 6.1 The construction of the final upper and lower blocks for a given skeleton } & 19\end{array}$

6.2 Physical and spurious graphs for a given configuration of the final upper and lower blocks 21

6.3 The sum over all physical and spurious graphs 24

7 Graph-based BCJ relation as a combination of traditional BCJ relations 29

$\begin{array}{lll}7.1 & \text { Examples } & 30\end{array}$

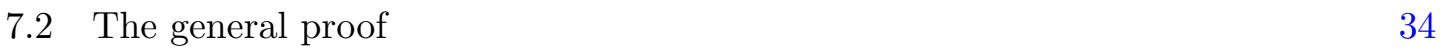

8 Gauge invariance identities of tree-level YM and GR amplitudes $\quad 38$

8.1 The old-version graphic rule for the BCJ numerators $n_{1|\zeta| n} \quad 39$

8.2 Understanding the gauge invariance induced identity eq. (8.5) 40

9 Conclusions and further discussions $\quad 45$

$\begin{array}{ll}\text { A Conventions and definitions } & 46\end{array}$ 
B More examples for section 4

B.1 The identity with $\mathbf{H}=\left\{h_{1}\right\} \quad 48$

B.2 The identity with $\mathbf{H}=\left\{h_{1}, h_{2}\right\} \quad 49$

B.3 The identity with $\mathbf{H}=\left\{h_{1}, h_{2}, h_{3}\right\} \quad 51$

C Graphs for the $\mathrm{H}=11$ example $\quad 55$

D General rules for the construction of physical graphs $\quad 55$

\section{Introduction}

Gauge invariance has been shown to provide a strong constraint on scattering amplitudes in recent years. It was not only used to solve amplitudes explicitly (see e.g., [1-4]) but also applied in understanding hidden structures of amplitudes. One interesting application is that a recursive expansion of single-trace Einstein-Yang-Mills (EYM) amplitudes can be determined by gauge invariance conditions of external gravitons in addition with a proper ansatz [5, 6] (the approach based on Cachazo-He-Yuan (CHY) formula [7-10] is provided in [11]). Applying the recursive expansion repeatedly, one expresses an arbitrary tree level single-trace EYM amplitude in terms of pure Yang-Mills (YM) ones whose coefficients are conveniently constructed by a graphic rule [12]. This expansion can be regarded as a generalization of the earlier studies on EYM amplitudes with few gravitons [13-16]. When the relation between gravity amplitudes and single-trace EYM amplitudes are further considered [5], we immediately expand any tree level gravity amplitude as a combination of Yang-Mills ones. It was shown that the recursive expansion for single-trace EYM amplitudes can be extended to all tree level multi-trace amplitudes via replacing some gravitons by gluon traces appropriately [17]. ${ }^{1}$

In the explicit form of the recursive expansion proposed in [5], gauge invariance conditions for all but one graviton (so-called 'fiducial graviton') are naturally encoded into the strength tensors as well as amplitudes with fewer gravitons. Nevertheless, the gauge invariance for the fiducial graviton does not manifest and further implies an nontrivial identity between EYM amplitudes. This identity plays as a consistency condition, which guarantees locality, in the Britto-Cachazo-Feng-Witten (BCFW) recursion approach $[18,19]$ to the expansion of EYM amplitudes [5]. Equivalently, if we take the gauge invariance condition of the fiducial graviton in the pure YM expansion, we immediately obtain a nontrivial identity for color-ordered YM amplitudes whose coefficients are polynomial functions of Lorentz inner products and depend on both polarizations of gravitons and external momenta. Similar discussions on generating relations for YM amplitudes by imposing gauge invariance can be found in $[1,3,6,14,17,20,21]$. An application of the gauge invariance induced identity is the proof of the equivalence between distinct approaches $[12,22,23]$ to scattering amplitudes in nonlinear sigma model [21].

\footnotetext{
${ }^{1}$ Discussions on multi-trace EYM amplitudes can also be found in [6, 14].
} 
Apart from the gauge invariance induced identity, color-ordered Yang-Mills amplitudes have also been shown to satisfy Kleiss-Kuijf (KK) [24] and Bern-Carrasco-Johansson (BCJ) relations [25], which reduce the number of independent amplitudes to $(n-3)$ ! (proofs can be found in [26-29]). A notable difference from the gauge invariance induced identity is that KK and BCJ relations do not include external polarizations in the coefficients. Thus it seems that the gauge invariance induced identity provides a new relation beyond $\mathrm{KK}$ and BCJ relations. However, direct evaluations of several examples $[5,6,17]$ imply that the gauge invariance induced identity is just a combination of BCJ relations. Due to the complexity of the coefficients in the expansion of EYM amplitudes, these examples provided in $[5,6,17]$ cannot be straightforwardly generalized to arbitrary case. Hence there is still lack of a systematical study on the full connection between the gauge invariance induced identity and BCJ relations.

In this paper, we investigate the relationship between an arbitrary gauge invariance induced identity, which is derived from the expansion of tree level single-trace EYM amplitudes or pure GR amplitudes, and general BCJ relations. We propose a 'refined graphic rule' in which different types of Lorentz inner products $\epsilon \cdot \epsilon, \epsilon \cdot k$ and $k \cdot k$, constructed by half polarizations of gravitons $\epsilon^{\mu}$ and external momenta $k^{\mu}$, are distinguished by different types of lines. Similar graphic rules have already been applied in the study of CHY formula (see $[30,31]$ ). By expressing the coefficients in the gauge invariance induced identity according to the refined graphic rule and collecting those terms with the same structure of the lines corresponding to coefficients $\epsilon \cdot \epsilon$ and $\epsilon \cdot k$ (such a structure is called a skeleton) in particular examples, we find that the gauge invariance induced identity can always be expressed by a combination of BCJ relations.

To generalize our observations to arbitrary case, more hidden structures of graphs should be revealed. We show that a skeleton of a graph corresponding to the gauge invariance induced identity always consists of no less than two maximally connected subgraphs (components) which are mutually disjoint with each other. Any physical graph, which agrees with the refined graphic rule, can be reconstructed by (i) first connecting these components (via $k \cdot k$ lines) into a graph with only two disjoint (called final upper and lower) blocks properly, (ii) then connecting the two disjoint blocks by a $k \cdot k$ line. For any given configuration of the final upper and lower blocks, spurious graphs are also introduced for convenience. We prove that the spurious graphs belonging to different configurations of the two disjoint blocks always cancel in pairs. Hence the sum over all physical graphs containing a same skeleton can be given by summing over both physical and spurious graphs with the same skeleton. A critical observation is that the total contribution of all physical and spurious graphs containing a same configuration of the final upper and lower blocks induces a graph-based BCJ relation which can be expanded in terms of traditional BCJ relations. As a consequence, the general gauge invariance induced identity from the expansion of tree level single-trace EYM amplitude is precisely expanded in terms of (traditional) BCJ relations. Similar discussions are applied to the gauge invariance induced identity of tree level GR amplitudes. The only notable difference is that the transversality of polarizations and momentum conservation in the pure gravity case should be taken into account. By the help of the language of CHY formulas [7-10], we further conclude that the 
gauge invariance induced identities of Yang-Mills-scalar (YMS) and pure YM amplitudes are combinations of BCJ relations for bi-scalar amplitudes.

The structure of this paper is following. In section 2, we review the expansion of tree level single-trace EYM amplitudes, the gauge invariance induced identity as well as traditional BCJ relations, which will be useful in the remaining sections. The refined graphic rule for the gauge invariance induced identity, which is derived from single-trace EYM amplitudes, as well as the main idea of this paper is provided in section 3. In section 4, direct evaluation of a typical example is presented. Inspired by this example (and examples presented in appendix), we provide discussions on the structure of skeletons in section 5 , the construction of all graphs for a given skeleton in section 6 and the graph-based BCJ relation in section 7 . The gauge invariance identities induced from pure gravity and YM amplitudes are discussed in section 8. We summarize this paper and provide some further discussions in section 9. Conventions of notations, more examples and the proof of the validity of the construction in section 6 are included in the appendix.

\section{Expansion of tree level single-trace EYM amplitudes, gauge invariance induced identity and traditional BCJ relation}

In this section, we review the recursive expansion of single-trace EYM amplitudes, the graphic rule, the gauge invariance induced identity from single-trace EYM amplitudes as well as the BCJ relations for Yang-Mills amplitudes.

\subsection{Recursive expansion of tree level single-trace EYM amplitudes}

Tree level single-trace EYM amplitude $A(1,2, \ldots, r \| \mathrm{H})$ with $r$ gluons $1,2, \ldots, r$ and $s$ gravitons $h_{1}, h_{2}, \ldots, h_{s}$ can be recursively expanded as (see [5])

$$
A(1,2, \ldots, r \| \mathrm{H})=\quad \sum_{\boldsymbol{h} \mid \tilde{\mathrm{h}} \boldsymbol{\sigma} \in\{2, \ldots, r-1\} \uplus\left\{\boldsymbol{h}, h_{a}\right\}} C_{h_{a}}(\boldsymbol{h}) A(1, \boldsymbol{\sigma}, r \| \widetilde{\mathrm{h}}),
$$

where the full graviton set $\left\{h_{1}, \ldots, h_{s}\right\}$ is denoted by $\mathrm{H}$ and an arbitrarily chosen $h_{a} \in \mathrm{H}$ is called fiducial graviton. The first summation on the r.h.s. is taken over all possible splittings of the set $\mathbf{H} \backslash h_{a} \rightarrow \boldsymbol{h} \mid \widetilde{\mathrm{h}}$ and all permutations of elements in $\boldsymbol{h}$ for a given splitting. For a fixed splitting $\mathrm{H} \backslash h_{a} \rightarrow \boldsymbol{h} \mid \widetilde{\mathrm{h}}$ and a fixed permutation of elements in $\boldsymbol{h}$, the second summation is taken over all the possible shuffle permutations $\boldsymbol{\sigma} \in\{2, \ldots, r-1\} \amalg\left\{\boldsymbol{h}, h_{a}\right\}$ (i.e. permutations in which the relative orders of elements in each set are kept). The coefficient $C_{h_{a}}(\boldsymbol{h})$ for a given splitting $\mathbf{H} \backslash h_{a} \rightarrow \boldsymbol{h} \mid \widetilde{\mathrm{h}}$, a given permutation $\left\{i_{1}, i_{2}, \ldots, i_{j}\right\}$ of elements in $\boldsymbol{h}$ and a given shuffle permutation $\boldsymbol{\sigma}$ is defined by

$$
C_{h_{a}}(\boldsymbol{h}) \equiv \epsilon_{h_{a}} \cdot F_{i_{j}} \cdots F_{i_{2}} \cdot F_{i_{1}} \cdot Y_{i_{1}}(\boldsymbol{\sigma})
$$

where the strength tensor $F_{i}^{\mu \nu}$ is

$$
F_{i}^{\mu \nu} \equiv k_{i}^{\mu} \epsilon_{i}^{\nu}-k_{i}^{\nu} \epsilon_{i}^{\mu}
$$


and $Y_{i_{1}}(\boldsymbol{\sigma})$ denotes the sum of all momenta of gluons $l \in\{1,2, \ldots, r-1\}$ s.t. $\boldsymbol{\sigma}^{-1}(l)<\boldsymbol{\sigma}^{-1}\left(i_{1}\right)$. Here the position of $i$ in the permutation $\sigma$ is denoted by $\sigma^{-1}(i) .^{2}$

\subsection{Graphic rule for the pure YM expansion of tree level single-trace EYM amplitudes}

Applying the recursive expansion (2.1) repeatedly, we finally express the single-trace EYM amplitude $A(1,2, \ldots, r \| \mathrm{H})$ in terms of pure $\mathrm{YM}$ ones

$$
A(1,2, \ldots, r \| \mathrm{H})=\quad \sum_{\boldsymbol{\sigma} \in\{2, \ldots, r-1\} \omega \operatorname{perms~} \mathrm{C}} \mathcal{C}(1, \boldsymbol{\sigma}, r) A(1, \boldsymbol{\sigma}, r) .
$$

In the above equation, we summed over all possible permutations $\sigma \in\{2, \ldots, r-1\} ш$ perms $\mathrm{H}$ in which the relative order of elements in $\{2, \ldots, r-1\}$ is preserved and perms $\mathrm{H}$ are all possible permutations of elements in $\mathrm{H}$. The coefficient $\mathcal{C}(1, \boldsymbol{\sigma}, r)$ for any permutation $\boldsymbol{\sigma}$ is given by

$$
\mathcal{C}(1, \boldsymbol{\sigma}, r)=\sum_{\mathcal{F} \in \mathcal{G}(1, \boldsymbol{\sigma}, r)} \mathcal{C}^{[\mathcal{F}]}
$$

where $\{\mathcal{G}(1, \boldsymbol{\sigma}, r)\}$ is used to denote the set of graphs $\mathcal{F}$ constructed by the following graphic rule:

(1) Define a reference order $\boldsymbol{\rho}$ of gravitons, then all $s$ gravitons are arranged into an ordered set

$$
\mathrm{R}=\left\{h_{\rho(1)}, h_{\rho(2)}, \ldots, h_{\rho(s)}\right\} .
$$

The position $\rho^{-1}(l)$ of $l(l \in \mathrm{H})$ in the ordered set $\mathrm{R}$ is called the weight of $l$. Apparently, $h_{\rho(s)}$ is the highest-weight node in R.

(2) Pick the highest-weight element $h_{\rho(s)}$ (the fiducial graviton for the first step recursive expansion) from the ordered set $\mathrm{R}$, an arbitrary gluon (root) $l \in\{1,2, \ldots, r-1\}$ as well as gravitons $i_{1}, i_{2}, \ldots, i_{j} \in \mathrm{H}$ s.t. $\sigma^{-1}(l)<\sigma^{-1}\left(i_{1}\right)<\sigma^{-1}\left(i_{2}\right)<\ldots \sigma^{-1}\left(i_{j}\right)<$ $\sigma^{-1}\left(h_{\rho(s)}\right)$. By considering each particle in the set $\left\{l, i_{1}, i_{2}, \ldots, i_{j}, h_{\rho(s)}\right\}$ as a node, we construct a chain $\mathbb{C H}=\left[h_{\rho(s)}, i_{j}, \ldots, i_{1}, l\right]$ which starts from the node $h_{\rho(s)}$ towards the node $l$. The graviton $h_{\rho(s)}$, the gluon $l$ and gravitons $i_{1}, i_{2}, \ldots, i_{j}$ are correspondingly mentioned as the starting node, the ending node and the internal nodes of this chain. We defined the weight of a chain by the weight of the starting node of the chain. The factor associated to this chain is

$$
\epsilon_{h_{\rho(s)}} \cdot F_{i_{j}} \cdot F_{i_{j-1}} \cdots F_{i_{1}} \cdot k_{l} .
$$

Redefine $\mathrm{R}$ by removing $i_{1}, i_{2}, \ldots, i_{j}, h_{\rho(s)}: \mathrm{R} \rightarrow \mathrm{R}^{\prime}=\mathrm{R} \backslash\left\{i_{1}, i_{2}, \ldots, i_{j}, h_{\rho(s)}\right\}$.

\footnotetext{
${ }^{2}$ Supposing that a given particle $i$ is located at the $m$-th position in the permutation $\sigma$, i.e. $i=\sigma(m)$, it is reasonable to define the position of $i$ in $\sigma$ by taking the 'inverse' of $\sigma$ on both sides, i.e., $m=\sigma^{-1}(i)$.
} 
(3) Picking $l^{\prime} \in\{1,2, \ldots, r-1\} \cup\left\{i_{1}, i_{2}, \ldots, i_{j}, h_{\rho(s)}\right\}$, the highest-weight element $h_{\rho^{\prime}\left(s^{\prime}\right)}$ (which is the fiducial graviton for the second step recursive expansion) in $\mathrm{R}^{\prime}$ as well as gravitons $i_{1}^{\prime}, i_{2}^{\prime}, \ldots, i_{j^{\prime}}^{\prime} \in \mathrm{R}^{\prime}$ s.t. $\sigma^{-1}\left(l^{\prime}\right)<\sigma^{-1}\left(i_{1}^{\prime}\right)<\sigma^{-1}\left(i_{2}^{\prime}\right)<\cdots<\sigma^{-1}\left(i_{j^{\prime}}^{\prime}\right)<$ $\sigma^{-1}\left(h_{\rho^{\prime}\left(s^{\prime}\right)}\right)$, we define a chain $\mathbb{C H}=\left\{h_{\rho^{\prime}\left(s^{\prime}\right)}, i_{j^{\prime}}^{\prime}, i_{j^{\prime}-1}^{\prime}, \ldots, i_{1}^{\prime}, l^{\prime}\right\}$ starting from $h_{\rho\left(s^{\prime}\right)}$ and ending at $l^{\prime}$. This chain is associated with a factor

$$
\epsilon_{\rho_{\rho^{\prime}\left(s^{\prime}\right)}} \cdot F_{i_{j^{\prime}}^{\prime}} \cdot F_{i_{j^{\prime}-1}^{\prime}} \cdots \cdots F_{i_{1}^{\prime}} \cdot k_{l^{\prime}}
$$

Remove $i_{1}^{\prime}, i_{2}^{\prime}, \ldots, i_{j^{\prime}}^{\prime}, h_{\rho^{\prime}\left(s^{\prime}\right)}$ from the ordered set $\mathrm{R}^{\prime}$ and redefine $\mathrm{R} \rightarrow \mathrm{R}^{\prime \prime}=\mathrm{R}^{\prime} \backslash$ $\left\{i_{1}^{\prime}, i_{2}^{\prime}, \ldots, i_{j^{\prime}}^{\prime}, h_{\rho^{\prime}\left(s^{\prime}\right)}\right\}$.

(4) Repeating the above steps until the ordered set $\mathrm{R}$ is empty, we obtain a graph $\mathcal{F}$ in which graviton trees are planted at gluons (roots) $\{1, \ldots, r-1\}$. For any given graph $\mathcal{F}$, the product of the factors accompanied to all chains produces a term $\mathcal{C}^{[\mathcal{F}]}$ in the coefficient $\mathcal{C}(1, \boldsymbol{\sigma}, r)$ in eq. (2.4). Thus the final expression of $\mathcal{C}(1, \boldsymbol{\sigma}, r)$ is given by summing over all possible graphs constructed by the above steps, i.e. eq. (2.5).

\subsection{Gauge invariance induced identity from tree level single-trace EYM amplitudes}

Gauge invariance requires that an EYM amplitude has to vanish when the 'half' graviton polarization $\epsilon_{h_{i}}$ is replaced by momenta $k_{h_{i}}$ for any $h_{i} \in \mathrm{H}$. Consequently, the pure-YM expansion eq. (2.4) should become zero under the replacement $\epsilon_{h_{i}} \rightarrow k_{h_{i}}$. Assuming that the half polarization $\epsilon_{h_{i}}$ is included in the coefficients in eq. (2.4), our discussion can be classified into the following two cases:

- If $h_{i}$ is the highest-weight element $h_{\rho(s)}$ (the fiducial graviton for the first-step expansion) in the reference order $\mathrm{R}$, it must be a starting node of some chain but cannot be an internal node of any chain. The gauge invariance condition for $h_{i}$ is not manifest and implies the following nontrivial relation for pure Yang-Mills amplitudes

$$
\left.\sum_{\boldsymbol{\sigma} \in\{2, \ldots, r-1\} \uplus \text { perms } \mathrm{H}} \mathcal{C}(1, \boldsymbol{\sigma}, r)\right|_{\epsilon_{\rho(s)} \rightarrow k_{h_{\rho(s)}}} A(1, \boldsymbol{\sigma}, r)=0,
$$

in which the coefficient $\left.\mathcal{C}(1, \boldsymbol{\sigma}, r)\right|_{\epsilon_{h_{\rho(s)}} \rightarrow k_{h}(s)}$ is obtained from eq. (2.5) via replacing $\epsilon_{h_{\rho(s)}}$ by $k_{h_{\rho(s)}}$. In other words, the chains led by $h_{\rho(s)}$ are of the form $k_{h_{\rho(s)}} \cdot F_{i_{j}} \cdots$ $F_{i_{2}} \cdot F_{i_{1}} \cdot k_{l}$.

- If $h_{i}$ is some graviton other than $h_{\rho(s)}$, it can be either an internal node or a starting node of some chain. The former case vanishes naturally because of the antisymmetry of the strength tensor $F_{h_{i}}^{\mu \nu}$. The latter case is achieved if the gauge invariance condition eq. (2.9) is already satisfied by amplitudes with fewer gravitons because in this case: (i) $h_{i}$ plays as the fiducial graviton for some intermediate-step recursive expansion in the graphic rule, and (ii) the sum over all the graphs, which contain the same chain structure produced by the preceding steps, is proportional to the l.h.s. of the gauge invariance condition (2.9) (with $h_{\rho(s)} \rightarrow h_{i}$ ) for fewer-graviton EYM amplitudes (Elements on chains produced by the preceding steps are considered as gluons). 
Since the gauge invariance condition for $h_{i} \neq h_{\rho(s)}$ is always achieved when the identity eq. (2.9) for fewer gravitons holds, our discussion can just be focused on the case with $h_{i}=h_{\rho(s)}$.

\subsection{BCJ relation}

Tree level color-ordered YM amplitudes have been proven to satisfy the following general BCJ relation (this general BCJ relation was introduced in [27, 29]):

$$
\sum_{\boldsymbol{\sigma} \in \boldsymbol{\beta} \amalg \boldsymbol{\alpha}} \sum_{l \in \boldsymbol{\beta}}\left(k_{l} \cdot X_{l}(\boldsymbol{\sigma})\right) A(1, \boldsymbol{\sigma}, r)=0,
$$

where $\beta$ and $\boldsymbol{\alpha}$ are two ordered sets of external gluons, $X_{l}(\boldsymbol{\sigma})$ denotes the sum of all momenta of gluons $a \in\{1, \sigma\} \cup \beta$ satisfying $\sigma^{-1}(a)<\sigma^{-1}(l)$ (the gluon 1 is always considered as the first one in the permutation $\boldsymbol{\sigma})$. In this paper, we the expression on the 1.h.s. of the eq. (2.10) is denoted as

$$
\mathcal{B}(1|\boldsymbol{\beta}, \boldsymbol{\alpha}| r)=\sum_{\boldsymbol{\sigma} \in \boldsymbol{\beta} \amalg \boldsymbol{\alpha}} \sum_{l \in \boldsymbol{\beta}}\left(k_{l} \cdot X_{l}(\boldsymbol{\sigma})\right) A(1, \boldsymbol{\sigma}, r) .
$$

\section{Refined graphic rule and the main idea}

In the previous section, we have shown that the gauge invariance condition for a singletrace EYM amplitude induces a nontrivial identity eq. (2.9) for pure Yang-Mills ones. The difference from BCJ relation is that coefficients in eq. (2.9) contain not only Mandelstam variables $k_{a} \cdot k_{b}$ but also other two types of Lorentz inner products $\epsilon_{a} \cdot \epsilon_{b}$ and $\epsilon_{a} \cdot k_{b}$ which involve half polarizations $\epsilon^{\mu}$. Such feature can be straightforwardly understood when all strength tensors $F_{i}^{\mu \nu}$ on both types of chains $\epsilon_{a} \cdot F_{i_{j}} \cdots \cdots F_{i_{2}} \cdot F_{i_{1}} \cdot k_{l}$ and $k_{\rho(s)} \cdot F_{i_{j}} \cdots \cdots F_{i_{2}} \cdot F_{i_{1}} \cdot k_{l}$ are expanded according to the definition (2.3). As shown by explicit examples in [5], the identity eq. (2.9) in fact can be expanded in terms of BCJ relations eq. (2.10) without referring any property of external polarizations $\epsilon_{i}^{\mu}$. Nevertheless, this observation cannot be trivially extended to the general identity eq. (2.9) because of the complexity of coefficients in eq. (2.9). Thus the relationship between the gauge invariance induced identity eq. (2.9) and BCJ relation eq. (2.10) is still unclear. In this section, we propose a refined graphic rule and show the main idea for studying the relationship between the identity eq. (2.9) and BCJ relation eq. (2.10), which will be helpful for our generic study in the coming sections.

\subsection{Refined graphic rule for single trace tree-level EYM amplitudes}

In the graphs constructed by the rule in section 2, the three types of Lorentz inner products $\epsilon_{a} \cdot \epsilon_{b}, \epsilon_{a} \cdot k_{b}$ and $k_{a} \cdot k_{b}$ cannot be distinguished. To investigate the general relationship between the gauge invariance induced identity (2.9) and BCJ relation eq. (2.10), we propose the following graphic rule by expanding all strength tensors $F_{a}^{\mu \nu}$ s.t. the three types of Lorentz inner products are represented by three distinct types of lines:

(1) Internal nodes. In the original graphic rule, each internal node stands for a strength tensor $F_{a}^{\mu \nu}$. When $F_{a}^{\mu \nu}$ is expanded into $k_{a}^{\mu} \epsilon_{a}^{\nu}-k_{a}^{\nu} \epsilon_{a}^{\mu}$, the corresponding internal node represents either $k_{a}^{\mu} \epsilon_{a}^{\nu}$ or $-k_{a}^{\nu} \epsilon_{a}^{\mu}$. Here the momentum $k_{a}^{\mu}$ and the 'half' polarization 


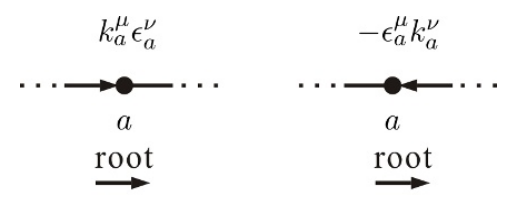

(a)

(b)

Figure 1. Two types of internal nodes of chains.

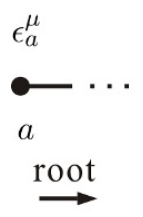

(a)

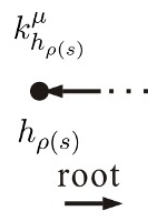

(b)

Figure 2. Two types of starting points of chains.

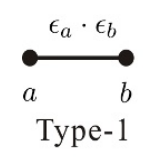

(a)

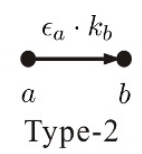

(b)

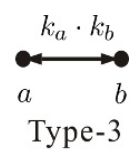

(c)

Figure 3. Three types of lines between nodes.

$\epsilon_{a}^{\nu}$ are respectively presented by an ingoing arrow line and an outcoming solid line. Then the strength tensor $F_{a}^{\mu \nu}$ becomes the sum of the two graphs in figure 1. As shown in figure 1 (a), we associate a plus with an arrow pointing to the direction of root. An arrow pointing away from root is associated with a minus (see figure 1 (b)).

(2) Starting nodes of chains. In the gauge invariance induced identity (2.9), each starting node of a chain is associated with either a 'half' polarization $\epsilon_{a}^{\mu}$ of some element other than $h_{\rho(s)}$ or the momentum $k_{h_{\rho(s)}}^{\mu}$ of the highest-weight element $h_{\rho(s)}$ in the ordered set R. Thus two distinct types of starting nodes are required. Noting that all chains are directed to roots, we introduce these two types of starting nodes figure 2 (a) and (b) by removing $(\ldots \cdot k)$ and $(-1)(\ldots \cdot \epsilon)$ from the internal nodes shown by figure 1 (a) and (b) respectively.

(3) Ending nodes of chains. Each internal/starting node of a chain or a root (the element in the original gluon set $\{1, \ldots, r\})$ can also be the ending node of another chain. The contraction of an ending node $b$ of some chain with its neighbor on the same chain always has the form $\left(\ldots k_{b}\right)$. Therefore, the ending node of a chain should be attached by a line of form $k_{a} \cdot k_{b}$ or $\epsilon_{a} \cdot k_{b}$.

(4) Three types of lines between nodes. Contractions of Lorentz indices are represented by connecting lines associated to nodes together. There are three distinct types of lines as shown by figure 3 (a) (type-1), (b) (type-2) and (c) (type-3) corresponding to the Lorentz inner products $\epsilon_{a} \cdot \epsilon_{b}, \epsilon_{a} \cdot k_{b}$ and $k_{a} \cdot k_{b}$. 


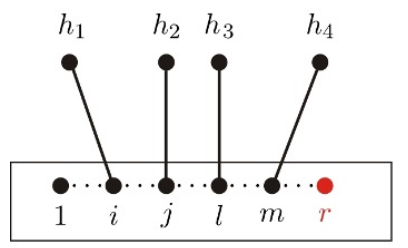

(a)

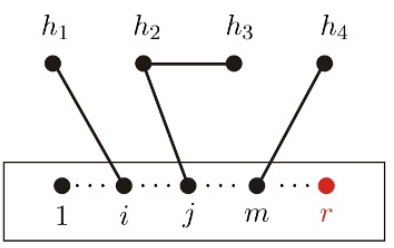

(b)

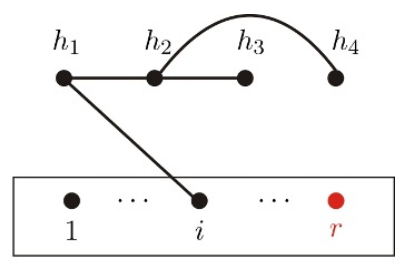

(c)

Figure 4. Examples of old-version graphs for the gauge invariance induced identity eq. (2.9). Here, the node $h_{4}$ denotes $k_{h_{4}}^{\mu}$, while the nodes $h_{1}, h_{2}$ and $h_{3}$ denote $\epsilon_{h_{1}}^{\mu}, \epsilon_{h_{2}}^{\mu}$ and $\epsilon_{h_{3}}^{\mu}$, respectively. We color the element $r$ by red to remind that the element $r$ always plays as the rightmost element in any permutation corresponding to a given graph. Only $1, \ldots, r-1$ can be roots.

With the above improvement, the coefficients $\left.\mathcal{C}(1, \boldsymbol{\sigma}, r)\right|_{\epsilon_{h_{\rho(s)} \rightarrow k_{h_{\rho(s)}}}}$ of the gauge invariance induced identity eq. (2.9) is then written by summing over all refined graphs. Thus eq. (2.9) becomes

$$
\sum_{\boldsymbol{\sigma} \in\{2, \ldots, r-1\}}\left[\sum_{\mathcal{F} \in \mathbb{G}(1, \boldsymbol{\sigma}, r)} \mathcal{D}^{[\mathcal{F}]}\right] A(1, \boldsymbol{\sigma}, r)=0,
$$

where $\mathbb{G}(1, \boldsymbol{\sigma}, r)$ denotes the set of all refined graphs which are allowed by the permutation $\{1, \boldsymbol{\sigma}, r\}$ according to the refined graphic rule. It is worth noting that a given graph $\mathcal{F}$ can be allowed by various permutations.

\subsubsection{Examples for the refined graphic rule}

Now we take the identity induced by the gauge invariance of the amplitude $A\left(1, \ldots, r \| h_{1}\right.$, $\left.h_{2}, h_{3}, h_{4}\right)$ as an example. We assume the reference order is $\mathrm{R}=\left\{h_{1}, h_{2}, h_{3}, h_{4}\right\}$ and consider the gauge invariance induced identity eq. (3.1) with $\epsilon_{h_{4}} \rightarrow k_{h_{4}}$.

Example-1. For any given permutation $\boldsymbol{\sigma} \in\{2, \ldots, r-1\} \amalg\left\{h_{1}\right\} \amalg\left\{h_{2}\right\} \amalg\left\{h_{3}\right\} \amalg\left\{h_{4}\right\}$, there must be old-version graphs figure 4 (a) contributing terms of the form $\left(k_{h_{4}} \cdot k_{m}\right)\left(\epsilon_{h_{3}}\right.$. $\left.k_{l}\right)\left(\epsilon_{h_{2}} \cdot k_{j}\right)\left(\epsilon_{h_{1}} \cdot k_{i}\right)(i, j, l, m \in\{1, \ldots, r-1\})$ to the coefficient $\left.\mathcal{C}(1, \sigma, r)\right|_{\epsilon_{h_{\rho(s)}} \rightarrow k_{h}(s)}$. The refined graph for such a coefficient is shown by figure 5 (a), in which each chain led by $h_{1}$, $h_{2}$ or $h_{3}$ consists of only one type- 2 line, while the chain led by $h_{4}$ is a type- 3 line.

Example-2. For any permutation $\boldsymbol{\sigma} \in\{2, \ldots, r-1\} \amalg\left\{h_{4}\right\} \amalg\left\{h_{2}, h_{3}\right\} \amalg\left\{h_{1}\right\}$, there exist old-version graphs figure 4 (b) consisting of three chains $\left(k_{h_{4}} \cdot k_{m}\right),\left(\epsilon_{h_{3}} \cdot F_{h_{2}} \cdot k_{j}\right)$ and $\left(\epsilon_{h_{1}} \cdot k_{i}\right)(i, j, m \in\{1, \ldots, r-1\})$. For given $i, j, m \in\{1, \ldots, r-1\}$, the graph figure 4 (b) contributes a term $\left(k_{h_{4}} \cdot k_{m}\right)\left(\epsilon_{h_{3}} \cdot F_{h_{2}} \cdot k_{j}\right)\left(\epsilon_{h_{1}} \cdot k_{i}\right)$ to $\left.\mathcal{C}(1, \boldsymbol{\sigma}, r)\right|_{\epsilon_{h_{\rho(s)}} \rightarrow k_{h_{\rho(s)}}}$. According to the refined graphic rule, this coefficient is given by summing the two graphs figure 5 (b) and (c) together:

$$
\text { (b) }:\left(k_{h_{4}} \cdot k_{m}\right)\left(\epsilon_{h_{3}} \cdot k_{h_{2}}\right)\left(\epsilon_{h_{2}} \cdot k_{j}\right)\left(k_{h_{1}} \cdot k_{i}\right), \quad(\mathrm{c}):(-1)\left(k_{h_{4}} \cdot k_{m}\right)\left(\epsilon_{h_{3}} \cdot \epsilon_{h_{2}}\right)\left(k_{h_{2}} \cdot k_{j}\right)\left(k_{h_{1}} \cdot k_{i}\right) \text {. }
$$




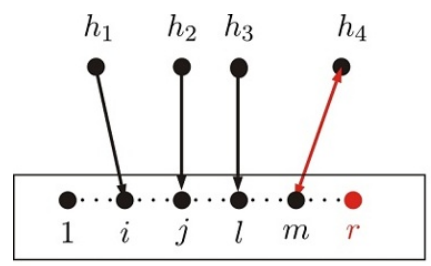

(a)

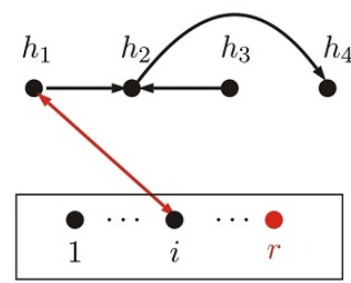

(d)

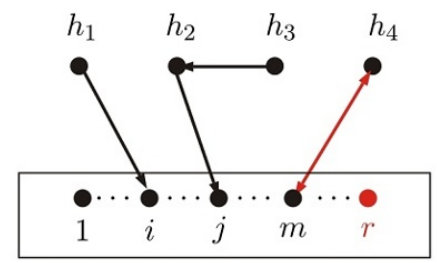

(b)

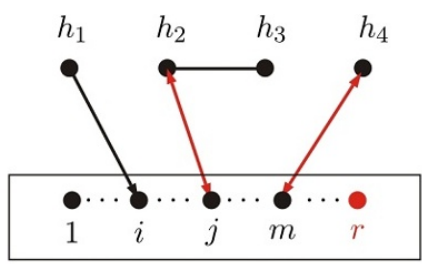

(c)

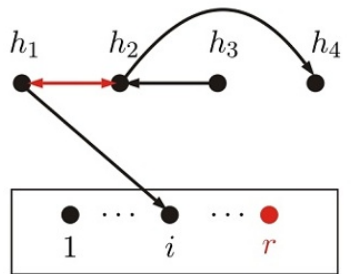

(e)

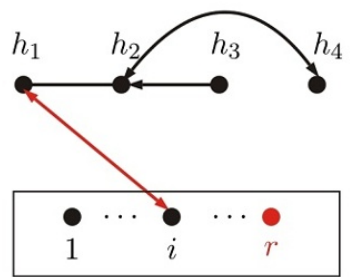

(f)

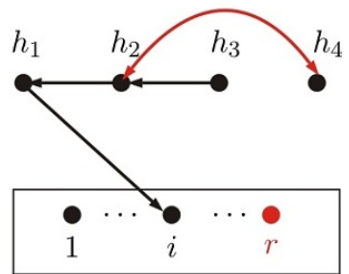

$(\mathrm{g})$

Figure 5. Graphs constructed by the refined graphic rule. The graph (a) corresponds to the old-version graph figure 4 (a), the graphs (b) and (c) correspond to the old-version graph figure 4 (b), while the graphs (d)-(g) correspond to the old-version graph figure 4 (c).

Example-3. For any permutation $\boldsymbol{\sigma} \in\{2, \ldots, r-1\} \amalg\left\{h_{1}, h_{2},\left\{h_{3}\right\} \amalg\left\{h_{4}\right\}\right\}$, there exist old-version graphs figure 4 (c) (for $i \in\{1, \ldots, r-1\}$ ) each of which contains two distinct chains $\left(k_{h_{4}} \cdot F_{h_{2}} \cdot F_{h_{1}} \cdot k_{i}\right)$ and $\left(\epsilon_{h_{3}} \cdot k_{h_{2}}\right)$. Thus the total contribution of such a graph is $\left(k_{h_{4}} \cdot F_{h_{2}} \cdot F_{h_{1}} \cdot k_{i}\right)\left(\epsilon_{h_{3}} \cdot k_{h_{2}}\right)$. According to the refined graphic rule, the coefficient for a given $i \in\{1, \ldots, r-1\}$ is provided by the sum of the four graphs figure 5 (d), (e), (f) and (g):
(d) $:(-1)^{2}\left(k_{h_{4}} \cdot \epsilon_{h_{2}}\right)\left(k_{h_{2}} \cdot \epsilon_{h_{1}}\right)\left(k_{h_{1}} \cdot k_{i}\right)\left(\epsilon_{h_{3}} \cdot k_{h_{2}}\right)$
(e) : $(-1)\left(k_{h_{4}} \cdot \epsilon_{h_{2}}\right)\left(k_{h_{2}} \cdot k_{h_{1}}\right)\left(\epsilon_{h_{1}} \cdot k_{i}\right)\left(\epsilon_{h_{3}} \cdot k_{h_{2}}\right)$
(f): $(-1)\left(k_{h_{4}} \cdot k_{h_{2}}\right)\left(\epsilon_{h_{2}} \cdot \epsilon_{h_{1}}\right)\left(k_{h_{1}} \cdot k_{i}\right)\left(\epsilon_{h_{3}} \cdot k_{h_{2}}\right)$
(g) : $\left(k_{h_{4}} \cdot k_{h_{2}}\right)\left(\epsilon_{h_{2}} \cdot k_{h_{1}}\right)\left(\epsilon_{h_{1}} \cdot k_{i}\right)\left(\epsilon_{h_{3}} \cdot k_{h_{2}}\right)$.

\subsection{The main idea}

Having established the refined graphic rule, we are now ready for studying the relationship between the gauge invariance induced identity eq. (3.1) and BCJ relation eq. (2.10). In the coming sections, we will prove that the gauge invariance induced identity eq. (3.1) can be expanded into a combination of BCJ relations. The main idea is the following:

Step-1. The factorization of coefficients. For any graph $\mathcal{F}$ constructed by the refined graphic rule, the coefficient $\mathcal{D}^{[\mathcal{F}]}$ can be factorized as a product of two coefficients $\mathcal{P}^{\left[\mathcal{F}^{\prime}\right]}$ and $\mathcal{K}^{\left[\mathcal{F} \backslash \mathcal{F}^{\prime}\right]}$ associated with a total factor $(-1)^{\mathcal{N}(\mathcal{F})}$. Here, the skeleton $\mathcal{F}^{\prime}$ is the subgraph which is obtained by deleting all type-3 lines from $\mathcal{F}$. The factor $\mathcal{P}^{\left[\mathcal{F}^{\prime}\right]}$ associated to the skeleton $\mathcal{F}^{\prime}$ contains only factors of forms $\left(\epsilon_{a} \cdot \epsilon_{b}\right)$ and $\left(\epsilon_{a} \cdot k_{b}\right)$. We use $\mathcal{F} \backslash \mathcal{F}^{\prime}$ to stand for the subgraph which is obtained by deleting all type- 1 and 2 lines from $\mathcal{F}$ (i.e. the complement of the skeleton). ${ }^{3}$ The factor $\mathcal{K}^{\left[\mathcal{F} \backslash \mathcal{F}^{\prime}\right]}$ corresponding to $\mathcal{F} \backslash \mathcal{F}^{\prime}$ contains only Mandelstam

\footnotetext{
${ }^{3}$ In this paper, ' $A \backslash B$ ' for a set $A$ and its subset $B$, if the sets are not considered as graphs, means we remove all elements of $\mathrm{B}$ from $\mathrm{A}$. For a graph $\mathcal{A}$ and its subgraph $\mathcal{B}$, the expression ' $\mathcal{A} \backslash \mathcal{B}$ ' means we remove all lines that are attached to nodes in $\mathcal{B}$ from $\mathcal{A}$ but keep the nodes. The expression ' $\mathcal{A}-\mathcal{B}$ ' is defined by removing all nodes in $\mathcal{B}$ and lines attached to these nodes from $\mathcal{A}$.
} 
variables $\left(k_{a} \cdot k_{b}\right)$. The total factor $(-1)^{\mathcal{N}(\mathcal{F})}$ depends on the number $\mathcal{N}(\mathcal{F})$ of arrows pointing away from the direction of roots (except the one connected to the highest-weight node $h_{\rho(s)}$ because we do not associate a minus to the arrow figure $\left.2(\mathrm{~b})\right)$. For instance, the factor $\mathcal{D}^{\left[\mathcal{F}^{\prime}\right]}$ for the graph figure $5(\mathrm{~d})$ is $(-1)^{2}\left(\epsilon_{h_{1}} \cdot k_{h_{2}}\right)\left(\epsilon_{h_{2}} \cdot k_{h_{4}}\right)\left(\epsilon_{h_{3}} \cdot k_{h_{3}}\right)\left(k_{h_{1}} \cdot k_{i}\right)$ which can be factorized into $\mathcal{P}^{\left[\mathcal{F}^{\prime}\right]}=\left(\epsilon_{h_{1}} \cdot k_{h_{2}}\right)\left(\epsilon_{h_{2}} \cdot k_{h_{4}}\right)\left(\epsilon_{h_{3}} \cdot k_{h_{3}}\right)$ and the factor $\mathcal{K}^{\left[\mathcal{F} \backslash \mathcal{F}^{\prime}\right]}=\left(k_{h_{1}} \cdot k_{i}\right)$ with a total sign $(-1)^{\mathcal{N}(\mathcal{F})}=(-1)^{2}$. With this factorization, the l.h.s. of the gauge invariance induced identity eq. (3.1) is expressed by

$$
T \equiv \sum_{\boldsymbol{\sigma} \in\{2, \ldots, r-1\} \omega \text { perms } \mathrm{H}}\left[\sum_{\mathcal{F} \in \mathbb{G}(1, \boldsymbol{\sigma}, r)}(-1)^{\mathcal{N}(\mathcal{F})} \mathcal{P}^{\left[\mathcal{F}^{\prime}\right]} \mathcal{K}^{\left[\mathcal{F} \backslash \mathcal{F}^{\prime}\right]}\right] A(1, \boldsymbol{\sigma}, r) .
$$

Here, we emphasize that a given skeleton $\mathcal{F}^{\prime}$ can belong to different permutations $\sigma \in$ $\{2, \ldots, r-1\} ш$ perms $\mathrm{H}$.

Step-2. Collecting all terms corresponding to the graphs $\mathcal{F} \supset \mathcal{F}^{\prime}, 4$ for any given skeleton $\mathcal{F}^{\prime}$. When all graphs containing the same skeleton are collected together, the expression eq. (3.4) becomes

$$
T=\sum_{\mathcal{F}^{\prime}} \mathcal{P}^{\left[\mathcal{F}^{\prime}\right]}\left[\sum_{\substack{\mathcal{F} \text { s.t. } \\ \mathcal{F} \mathcal{F}^{\prime}}}(-1)^{\mathcal{N}(\mathcal{F})} \mathcal{K}^{\left[\mathcal{F} \backslash \mathcal{F}^{\prime}\right]} \sum_{\boldsymbol{\sigma}^{\mathcal{F}}} A\left(1, \boldsymbol{\sigma}^{\mathcal{F}}, r\right)\right]
$$

where we summed over (i) all possible skeletons $\mathcal{F}^{\prime}$, (ii) all possible graphs $\mathcal{F}$ which are constructed by the refined graphic rule and satisfy $\mathcal{F} \supset \mathcal{F}^{\prime}$ for a given skeleton $\mathcal{F}^{\prime}$, (iii) all permutations $\boldsymbol{\sigma}^{\mathcal{F}}$ for a given $\mathcal{F}$.

Step-3. Finding out the relationship between terms associated with a skeleton and the l.h.s. of BCJ relations. For any given skeleton $\mathcal{F}^{\prime}$ in eq. (3.5), we will prove that the expression in the square brackets can be written in terms of the l.h.s. of BCJ relations.

We first present direct evaluations of simple examples in the next section and extract common features which will be systematically studied in sections 5, 6 and 7 .

\section{Direct evaluations}

In this section, we verify that the expression in the square brackets in eq. (3.5) can be expanded in terms of the 1.h.s. of BCJ relations, through an explicit example. Some common features that will be useful for the general proof are further extracted. More examples with $|\mathrm{H}| \leq 3$ are included in appendix B.

\subsection{A typical example}

When $\mathbf{H}=\left\{h_{1}, h_{2}, h_{3}\right\}$ and the reference order is chosen as $\mathbf{R}=\left\{h_{1}, h_{2}, h_{3}\right\}$, the expression eq. (3.5) has nine skeletons, all of them are displayed in figure 6 . We now consider the skeleton $\mathscr{F}^{\prime}=$ figure 6 (i) and calculate the expression in the square brackets in eq. (3.5)

\footnotetext{
${ }^{4}$ In this paper, when we write $\mathcal{F} \supset \mathcal{F}^{\prime}$ or $\mathcal{F}^{\prime} \subset \mathcal{F}$, we always mean that $\mathcal{F}^{\prime}$ is not only a subset of the graph $\mathcal{F}$ but also a skeleton.
} 
explicitly. We will find that the sum over all possible graphs $\mathscr{F}$ satisfying $\mathscr{F} \supset \mathscr{F}^{\prime}$ in the square brackets of eq. (3.5) can be written as a proper combination of BCJ relations.

The skeleton figure 6 (i) consists of three mutually disjoint components which correspondingly contain all elements of $\{1, \ldots, r\}$, the single node $h_{3}$ and the subgraph with nodes $h_{1}$ and $h_{2}$. The factor $\mathcal{P}^{\left[\mathcal{F}^{\prime}\right]}$ for figure 6 (i) is $\epsilon_{h_{1}} \cdot \epsilon_{h_{2}}$ and all possible graphs $\mathcal{F} \supset \mathcal{F}^{\prime}=$ figure 6 (i) are shown by figure 7 (a), (b), (d) and (e). For a given graph figure 7 (a) or (b), in which $h_{1}$ is contracted with an arbitrary $l \in\{1, \ldots, r-1\}$, the possible relative orders $\beta$ for elements in $\left\{h_{1}, h_{2}\right\} \sqcup\{l+1, \ldots, r-1\}$ satisfy

$$
\beta \in\left\{1, \ldots, l,\left\{h_{1}, h_{2}\right\} ш\{l+1, \ldots, r-1\}\right\} .
$$

Hence all permutations contributing to graphs figure 7 (a) and (b) have the form $\sigma \in$ $\left\{h_{3}\right\} ш \beta$. To get the total contribution of all graphs figure 7 (a) and (b), we should collect coefficients $(-1)^{\mathcal{N}(\mathcal{F})} \mathcal{K}^{\left[\mathcal{F} \backslash \mathcal{F}^{\prime}\right]}$ for $A(1, \boldsymbol{\sigma}, r)$ with a given permutation $\boldsymbol{\sigma} \in\left\{h_{3}\right\} ш \boldsymbol{\beta}$ (see table 1), then sum over all $\sigma \in\left\{h_{3}\right\} ш \beta$, all $\beta$ satisfying eq. (4.1) and all $l \in\{1, \ldots, r-1\}$. The total contribution of all graphs figure 7 (a) and (b) thus is written as

$$
\begin{aligned}
T_{1}= & -\sum_{l \in\{1, \ldots, r-1\}}\left(k_{h_{1}} \cdot k_{l}\right) \sum_{\substack{\boldsymbol{\beta} \in\left\{2, \ldots, l,\left\{h_{1}, h_{2}\right\} \\
\uplus\{l+1, \ldots, r-1\}\right\}}}\left[\sum_{\substack{\boldsymbol{\sigma} \in\left\{h_{3}\right\} \uplus \beta \\
\boldsymbol{\sigma}^{-1}\left(h_{2}\right)<\boldsymbol{\sigma}^{-1}\left(h_{3}\right)}}\left(k_{h_{3}} \cdot Y_{h_{3}}(\boldsymbol{\sigma})+k_{h_{3}} \cdot k_{h_{2}}\right) A(1, \boldsymbol{\sigma}, r)\right. \\
& \left.+\sum_{\substack{\boldsymbol{\sigma} \in\left\{h_{3}\right\} \uplus \boldsymbol{\beta} \\
\boldsymbol{\sigma}^{-1}\left(h_{1}\right)<\boldsymbol{\sigma}^{-1}\left(h_{3}\right)<\boldsymbol{\sigma}^{-1}\left(h_{2}\right)}}\left(k_{h_{3}} \cdot Y_{h_{3}}(\boldsymbol{\sigma})\right) A(1, \boldsymbol{\sigma}, r)+\sum_{\substack{\boldsymbol{\sigma} \in\left\{h_{3}\right\} \uplus \boldsymbol{\beta} \\
\boldsymbol{\sigma}^{-1}\left(h_{3}\right)<\boldsymbol{\sigma}^{-1}\left(h_{1}\right)}}\left(k_{h_{3}} \cdot Y_{h_{3}}(\boldsymbol{\sigma})\right) A(1, \boldsymbol{\sigma}, r)\right] .
\end{aligned}
$$

For a given graph figure 7 (d) or (e), where $h_{2}$ and $h_{3}$ are respectively contracted with any $l \in\{1, \ldots, r-1\}$, all the possible permutations $\sigma$ contributing to figure 7 (d) and (e) satisfy

$$
\begin{aligned}
& \boldsymbol{\sigma} \in\{1, \ldots, r-1\} \amalg\left\{h_{2}, h_{1}, h_{3}\right\}, \text { for Fig. } 7 \text { (d), } \\
& \boldsymbol{\sigma} \in\{1, \ldots, r-1\} \amalg\left\{h_{3}, h_{1}, h_{2}\right\}, \text { for Fig. } 7 \text { (e). }
\end{aligned}
$$

When collecting the coefficients $(-1)^{\mathcal{N}(\mathcal{F})} \mathcal{K}^{\left[\mathcal{F} \backslash \mathcal{F}^{\prime}\right]}$ for any given $\boldsymbol{\sigma}$ (see table 1) and summing over all possible $\boldsymbol{\sigma}$, we arrive the total contribution of the graphs figure 7 (d) and (e)

$$
T_{2}=-\left(k_{h_{1}} \cdot k_{h_{3}}\right)\left[\sum_{\substack{\boldsymbol{\sigma} \in\{2, \ldots, r-1\} \\ \uplus\left\{h_{3}, h_{1}, h_{2}\right\}}}\left(k_{h_{3}} \cdot Y_{h_{3}}(\boldsymbol{\sigma})\right) A(1, \boldsymbol{\sigma}, r)+\sum_{\substack{\boldsymbol{\sigma} \in\{2, \ldots, r-1\} \\ \uplus\left\{h_{2}, h_{1}, h_{3}\right\}}}\left(k_{h_{2}} \cdot Y_{h_{2}}(\boldsymbol{\sigma})\right) A(1, \boldsymbol{\sigma}, r)\right] .
$$

In order to reorganize $T_{1}+T_{2}$ in terms of the l.h.s. of BCJ relations, we introduce so-called spurious graphs figure 7 (c) and (f) which also contains the skeleton figure 6 (i) but are not real graphs constructed by the refined graphic rule. The two spurious graphs figure 7 (c) and (f) have the same structure with opposite signs, thus they must cancel with one another. Relative orders of $h_{1}, h_{2}$ and $h_{3}$ in a spurious graph figure 7 (c) or (f) 


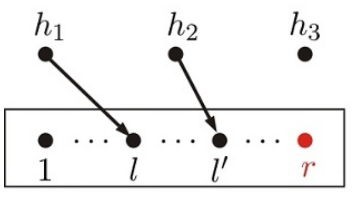

(a)

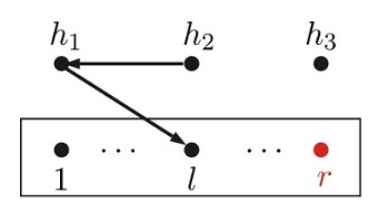

(d)

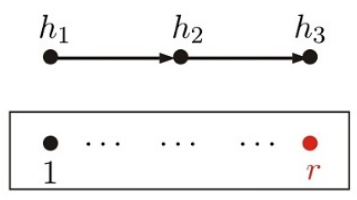

$(\mathrm{g})$

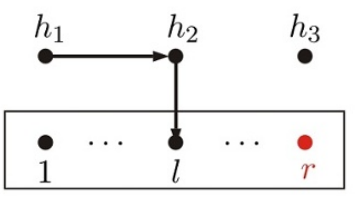

(b)

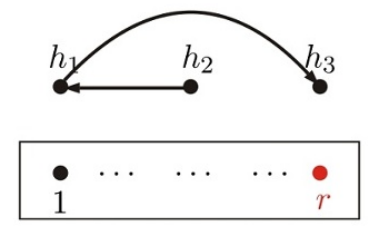

(e)

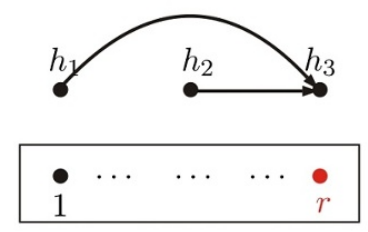

(h)

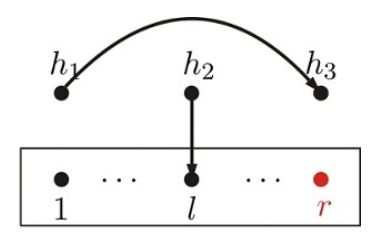

(c)

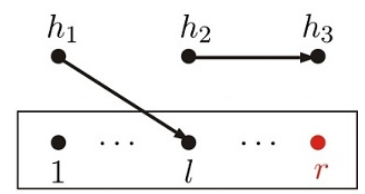

(f)

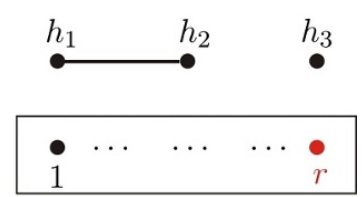

(i)

Figure 6. All skeletons for the gauge invariance induced identity with $\mathbf{H}=\left\{h_{1}, h_{2}, h_{3}\right\}$.

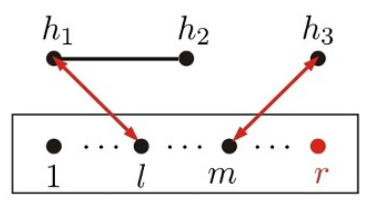

(a)

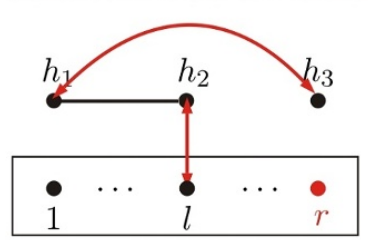

(d)

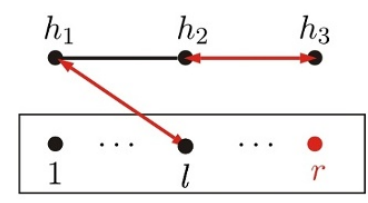

(b)

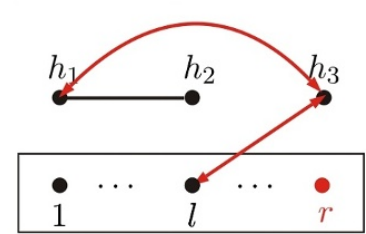

(e)

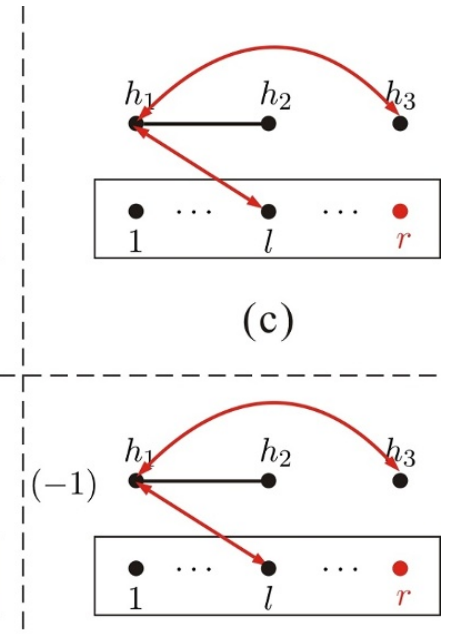

(c)

(f)

Figure 7. Graphs (a), (b), (d) and (e) are physical graphs, which are constructed by the refined graphic rule, for the skeleton $\mathcal{F}^{\prime}=$ figure 6 (i). Graphs (d) and (e) are spurious graphs for the skeleton $\mathcal{F}^{\prime}=$ figure 6 (i). All spurious graphs cancel with each other. 


\begin{tabular}{|c|c|c|c|c|}
\hline $\begin{array}{l}\text { Relative orders } \\
\text { of } h_{1}, h_{2} \text { and } h_{3}\end{array}$ & $\begin{array}{c}\text { Physical graphs } \\
\qquad \mathcal{F} \supset \mathcal{F}^{\prime}\end{array}$ & $\begin{array}{c}(-1)^{\mathcal{N}(\mathcal{F})} \mathcal{K}^{\left[\mathcal{F} \backslash \mathcal{F}^{\prime}\right]} \text { from } \\
\text { physical graphs }\end{array}$ & $\begin{array}{l}\text { Spurious graphs } \\
\qquad \mathcal{F} \supset \mathcal{F}^{\prime}\end{array}$ & $\begin{array}{c}\text { Factors from } \\
\text { spurious graphs }\end{array}$ \\
\hline$\left\{h_{1}, h_{2}, h_{3}\right\}$ & Fig. 7 (a),(b) & $-\left(k_{h_{1}} \cdot k_{l}\right)\left(k_{h_{3}} \cdot Y_{h_{3}}+k_{h_{3}} \cdot k_{h_{2}}\right)$ & Fig. 7 (c) & $-\left(k_{h_{3}} \cdot k_{h_{1}}\right)\left(k_{h_{1}} \cdot k_{l}\right)$ \\
\hline$\left\{h_{1}, h_{3}, h_{2}\right\}$ & Fig. 7 (a) & $-\left(k_{h_{1}} \cdot k_{l}\right)\left(k_{h_{3}} \cdot Y_{h_{3}}\right)$ & Fig. 7 (c) & $-\left(k_{h_{3}} \cdot k_{h_{1}}\right)\left(k_{h_{1}} \cdot k_{l}\right)$ \\
\hline$\left\{h_{3}, h_{1}, h_{2}\right\}$ & Fig. 7 (a) & $-\left(k_{h_{1}} \cdot k_{l}\right)\left(k_{h_{3}} \cdot Y_{h_{3}}\right)$ & no & 0 \\
\hline$\left\{h_{1}, h_{2}, h_{3}\right\}$ & no & 0 & Fig. 7 (f) & $\left(k_{h_{3}} \cdot k_{h_{1}}\right)\left(k_{h_{1}} \cdot Y_{h_{1}}\right)$ \\
\hline$\left\{h_{1}, h_{3}, h_{2}\right\}$ & no & 0 & Fig. 7 (f) & $\left(k_{h_{3}} \cdot k_{h_{1}}\right)\left(k_{h_{1}} \cdot Y_{h_{1}}\right)$ \\
\hline$\left\{h_{2}, h_{1}, h_{3}\right\}$ & Fig. 7 (d) & $-\left(k_{h_{2}} \cdot Y_{h_{2}}\right)\left(k_{h_{3}} \cdot k_{h_{1}}\right)$ & no & 0 \\
\hline$\left\{h_{3}, h_{1}, h_{2}\right\}$ & Fig. 7 (e) & $-\left(k_{h_{1}} \cdot k_{h_{3}}\right)\left(k_{h_{3}} \cdot Y_{h_{3}}\right)$ & no & 0 \\
\hline
\end{tabular}

Table 1. For a given $l$ and a given $\beta$ satisfying eq. (4.1) in the upper part of figure 7, possible relative orders of elements $h_{1}, h_{2}$ and $h_{3}$ are $\left\{h_{1}, h_{2}, h_{3}\right\},\left\{h_{1}, h_{3}, h_{2}\right\}$ and $\left\{h_{3}, h_{1}, h_{2}\right\}$. The corresponding (physical and spurious) graphs and factors are collected on the second, third and fourth rows (Recalling that the definition of $Y_{h}(\boldsymbol{\sigma})$ is $\left.Y_{h}(\boldsymbol{\sigma})=\sum_{\sigma^{-1}(i)<\sigma^{-1}(h)} k_{i}\right)$. Relative orders for graphs in the lower part of figure 7 and their factors are collected on the last four rows.

can be $\left\{h_{1}, h_{2}, h_{3}\right\}$ and $\left\{h_{1}, h_{3}, h_{2}\right\}$. The spurious graphs corresponding to figure 7 (c) for all $l$ contribute (see table 1 )

$$
\begin{aligned}
& -\left(k_{h_{3}} \cdot k_{h_{1}}\right)\left[\sum_{l \in\{1, \ldots, r-1\}}\left(k_{h_{1}} \cdot k_{l}\right) \sum_{\substack{\boldsymbol{\sigma} \in\left\{2, \ldots, l,\left\{h_{1},\left\{h_{2}\right\} \uplus\left\{h_{3}\right\}\right\} \\
w\{l+1, \ldots, r-1\}\right\}}} A(1, \boldsymbol{\sigma}, r)\right] \\
& =-\left(k_{h_{3}} \cdot k_{h_{1}}\right)\left[\sum_{\substack{\boldsymbol{\sigma} \in\left\{h_{1},\left\{h_{2}\right\} \uplus\left\{h_{3}\right\}\right\} \\
\uplus\{2, \ldots, r-1\}}}\left(k_{h_{1}} \cdot Y_{h_{1}}\right) A(1, \boldsymbol{\sigma}, r)\right],
\end{aligned}
$$

to the skeleton $\mathcal{F}^{\prime}=$ figure 6 (i), while the spurious graphs corresponding to figure 7 (f) have the same contribution but with an opposite sign.

For any given $l$ on the l.h.s. of eq. (4.5), the sum over $\sigma$ can be achieved by first summing over all permutations $\boldsymbol{\sigma} \in\left\{h_{3}\right\} ш \boldsymbol{\beta}$ which satisfy $\boldsymbol{\sigma}^{-1}\left(h_{2}\right)<\boldsymbol{\sigma}^{-1}\left(h_{3}\right)$ or $\boldsymbol{\sigma}^{-1}\left(h_{1}\right)<$ $\sigma^{-1}\left(h_{3}\right)<\sigma^{-1}\left(h_{2}\right)$ for any given $\beta$ satisfying eq. (4.1), then summing over all possible $\beta$ satisfying eq. (4.1). Therefore, the sum of all contributions from the spurious graph figure 7 (c) and the physical graphs figure 7 (a), (b) is given by the sum of eq. (4.5) and $T_{1}$ :

$$
\begin{aligned}
& -\sum_{l \in\{1, \ldots, r-1\}}\left(k_{h_{1}} \cdot k_{l}\right) \sum_{\substack{\boldsymbol{\beta} \in\left\{2, \ldots, l,\left\{h_{1}, h_{2}\right\} \\
\uplus\{l+1, \ldots, r-1\}\right\}}}\left[\sum_{\substack{\boldsymbol{\sigma} \in\left\{h_{3}\right\} \uplus \beta \\
\boldsymbol{\sigma}^{-1}\left(h_{2}\right)<\boldsymbol{\sigma}^{-1}\left(h_{3}\right)}}\left(k_{h_{3}} \cdot Y_{h_{3}}(\boldsymbol{\sigma})+k_{h_{3}} \cdot k_{h_{2}}+k_{h_{3}} \cdot k_{h_{1}}\right) A(1, \boldsymbol{\sigma}, r)\right. \\
& \left.+\sum_{\substack{\boldsymbol{\sigma} \in\left\{h_{3}\right\} \uplus \boldsymbol{\beta} \\
\boldsymbol{\sigma}^{-1}\left(h_{1}\right)<\boldsymbol{\sigma}^{-1}\left(h_{3}\right)<\boldsymbol{\sigma}^{-1}\left(h_{2}\right)}}\left(k_{h_{3}} \cdot Y_{h_{3}}(\boldsymbol{\sigma})+k_{h_{3}} \cdot k_{h_{1}}\right) A(1, \boldsymbol{\sigma}, r)+\sum_{\substack{\boldsymbol{\sigma} \in\left\{h_{3}\right\} \uplus \boldsymbol{\beta} \\
\boldsymbol{\sigma}^{-1}\left(h_{3}\right)<\boldsymbol{\sigma}^{-1}\left(h_{1}\right)}}\left(k_{h_{3}} \cdot Y_{h_{3}}(\boldsymbol{\sigma})\right) A(1, \boldsymbol{\sigma}, r)\right] \\
& =-\sum_{l \in\{1, \ldots, r-1\}}\left(k_{h_{1}} \cdot k_{l}\right) \sum_{\substack{\boldsymbol{\beta} \in\left\{2, \ldots, l,\left\{h_{1}, h_{2}\right\} \\
w\{l+1, \ldots, r-1\}\right\}}} \mathcal{B}\left(1\left|\left\{h_{3}\right\}, \boldsymbol{\beta}\right| r\right) .
\end{aligned}
$$




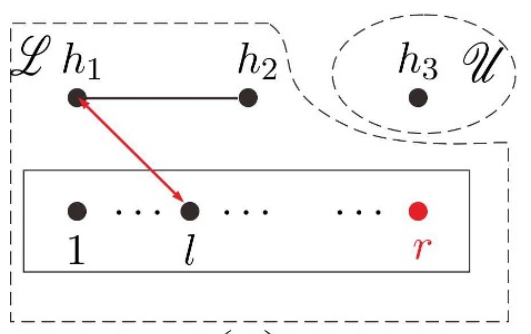

(a)

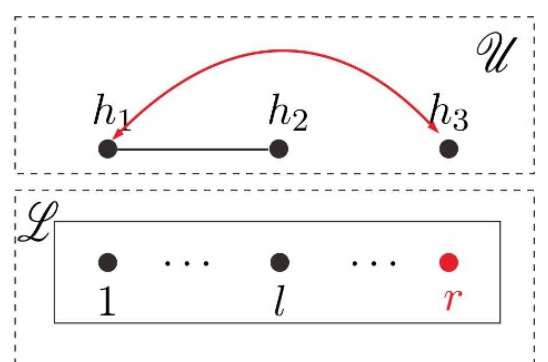

(b)

Figure 8. Graphs (a) and (b) are the possible configurations of the final upper and lower blocks $\mathscr{U}$, for the skeleton figure $6(\mathrm{i})$.

The sum of the contributions from the spurious graph figure 7 (f) and the physical graphs figure 7 (d), (e) is given by the sum of eq. (4.5) (here we make use of the r.h.s. of eq. (4.5) and notice that there is an extra minus) and $T_{2}$ :

$$
\begin{aligned}
\left(k_{h_{1}} \cdot k_{h_{3}}\right)\left[-\sum_{\substack{\boldsymbol{\sigma} \in\{2, \ldots, r-1\} \\
\uplus\left\{h_{3}, h_{1}, h_{2}\right\}}}\left(k_{h_{3}} \cdot Y_{h_{3}}(\boldsymbol{\sigma})\right) A(1, \boldsymbol{\sigma}, r)-\sum_{\substack{\boldsymbol{\sigma} \in\{2, \ldots, r-1\} \\
\uplus\left\{h_{2}, h_{1}, h_{3}\right\}}}\left(k_{h_{2}} \cdot Y_{h_{2}}(\boldsymbol{\sigma})\right) A(1, \boldsymbol{\sigma}, r)\right. \\
\left.+\sum_{\substack{\boldsymbol{\sigma} \in\{2, \ldots, r-1\} \\
\uplus\left\{h_{1},\left\{h_{2}\right\} \uplus\left\{h_{3}\right\}\right\}}}\left(k_{h_{1}} \cdot Y_{h_{1}}(\boldsymbol{\sigma})\right) A(1, \boldsymbol{\sigma}, r)\right]
\end{aligned}
$$

When we add the following two terms

$$
-\sum_{\substack{\boldsymbol{\sigma} \in\{2, \ldots, r-1\} \\ \uplus\left\{h_{1},\left\{h_{2}\right\} \uplus\left\{h_{3}\right\}\right\}}}\left(k_{h_{3}} \cdot X_{h_{3}}(\boldsymbol{\sigma})\right) A(1, \boldsymbol{\sigma}, r) \text { and }-\sum_{\substack{\boldsymbol{\sigma} \in\{2, \ldots, r-1\} \\ \uplus\left\{h_{1},\left\{h_{2}\right\} \uplus\left\{h_{3}\right\}\right\}}}\left(k_{h_{2}} \cdot X_{h_{2}}(\boldsymbol{\sigma})\right) A(1, \boldsymbol{\sigma}, r)
$$

to the first and the second term of eq. (4.7) respectively and subtract them from the third term of eq. (4.7), the expression (4.7) turns to the following combination of the l.h.s. of BCJ relations

$$
\begin{aligned}
\left(k_{h_{1}} \cdot k_{h_{3}}\right) & {\left[-\sum_{\substack{\boldsymbol{\beta} \in\{2, \ldots, r-1\} \\
\omega\left\{h_{1}, h_{2}\right\}}} \mathcal{B}\left(1\left|\left\{h_{3}\right\}, \boldsymbol{\beta}\right| r\right)-\sum_{\substack{\boldsymbol{\beta} \in\{2, \ldots, r-1\} \\
\omega\left\{h_{1}, h_{3}\right\}}} \mathcal{B}\left(1\left|\left\{h_{2}\right\}, \boldsymbol{\beta}\right| r\right)\right.} \\
& \left.+\sum_{\boldsymbol{\rho} \in\left\{h_{1},\left\{h_{2}\right\} \uplus\left\{h_{3}\right\}\right\}} \mathcal{B}(1|\boldsymbol{\rho},\{2, \ldots, r-1\}| r)\right] .
\end{aligned}
$$

Finally, the sum of $T_{1}$ and $T_{2}$ becomes the sum of eq. (4.6) and eq. (4.8) which are already expanded by the 1.h.s. of BCJ relations.

\subsection{Common features of the examples}

Now we extract some common features from the examples given in section 4.1 and appendix B. 
(1). Structure of skeletons $\mathcal{F}^{\prime}$. As shown by the example in section 4.1 and the examples given in appendix $\mathrm{B}$, each skeleton consists of at least two mutually disjoint components: the one containing the highest-weight graviton $h_{\rho(s)}$ (type-II component) and the one containing all elements in $\{1, \ldots, r\}$ (type-III component). Other components (type-I components), each of which always involves a type- 1 line, may also appear in a skeleton (see figure 6 (i)).

(2). The final upper and lower blocks. For any given skeleton, one can connect all its components into only two disjoint blocks properly: one block contains the highest-weight graviton $h_{\rho(s)}$ and is called the final upper block $\mathscr{U}$, the other block contains the elements in $\{1, \ldots, r\}$ and is called the final lower block $\mathscr{L}$. Apparently, if a skeleton has only two disjoint components (as the examples in appendix B), the final upper and lower blocks are just the type-II and the type-III components. For skeletons with at least three components (as the example shown in section 4.1), one should connect the type-I components towards either the type-II component or the type-III component properly, via type-3 lines. After all type-I components have been considered, the skeleton becomes a graph having only two disjoint parts which correspond to the final upper and lower components (see figure 8).

(3). Physical and spurious graphs. For a given configuration of final upper and lower blocks $\mathscr{U}, \mathscr{L}$, we need to connect $\mathscr{U}$ and $\mathscr{L}$ together by a type-3 line. If the original skeleton consists of only two components (as the examples in appendix B), all graphs allowed by the refined graphic rule are obtained by connecting any node $a \in \mathscr{U}$ and $b \in \mathscr{L} \backslash\{r\}$ via a type-3 line. If the original skeleton consists of at least three components, one should be more careful: a graph produced by connecting a type three line between $\mathscr{U}$ and $\mathscr{L} \backslash\{r\}$ may be either physical (in agreement with the refined graphic rule, e.g. figure 7 (a), (b), (d) and (e)) or spurious (not allowed by the graphic rule e.g. figure 7 (c) and (f)). However, one can associate proper signs to the spurious graphs s.t. all spurious graphs cancel in pairs (as the cancelation between figure 7 (c) and (f)). Thus the total contribution for a given configuration of the final upper and lower blocks can also be given by summing over all those graphs produced by connecting any node $a \in \mathscr{U}$ and $b \in \mathscr{L} \backslash\{r\}$ via a type-3 line.

(4). The sum of all (physical and spurious) graphs for a given configuration of the final upper and lower blocks. The sum of all (physical and spurious) graphs corresponding to a given final upper and lower blocks can be written as a combination of the 1.h.s. of BCJ relations.

In the coming sections, we generalize the above observations to arbitrary cases. In section 5, we prove the general structure of skeletons observed in (1). In section 6 , we provide the general rule for constructing graphs corresponding to a given skeleton (as stated by the points (2) and (3)): first construct all final upper and lower blocks $\mathscr{U}, \mathscr{L}$, then construct all physical and spurious graphs for a given configuration of $\mathscr{U}, \mathscr{L}$. The contributions from all (physical and spurious) graphs for given $\mathscr{U}$ and $\mathscr{L}$ can be arranged as a proper combination of the l.h.s. of BCJ relations, due to a so-called graph-based BCJ relation which will be proved in section 7 . 


\section{$5 \quad$ General structure of skeletons}

To study the general pattern of a skeleton, we recall that there are two kinds of chains in a graph (defined by the old-version graphic rule): (i) a chain led by any node $a$ in $\mathbf{H} \backslash\left\{h_{\rho(s)}\right\}$ has the form $\epsilon_{a} \cdot F_{i_{j}} \cdot \ldots \cdot F_{i_{1}} \cdot k_{b}$ (here $b$ can be either a node on a higher-weight chain or an element of $\{1, \ldots, r-1\}$ ), (ii) the chain led by the highest weight element $h_{\rho(s)}$ has the form $k_{h_{\rho(s)}} \cdot F_{i_{j}} \cdot \ldots \cdot F_{i_{1}} \cdot k_{b}(b \in\{1, \ldots, r-1\})$ because we have replaced $\epsilon_{h_{\rho(s)}}$ by $k_{h_{\rho(s)}}$ in the expansion of EYM amplitude. We shall study the structures of these two types of chains in turn:

(i) When all strength tensors $F^{\mu \nu}$ are expressed by its definition eq. (2.3), a chain $\epsilon_{a} \cdot F_{i_{j}} \cdot \ldots \cdot F_{i_{2}} \cdot F_{i_{1}} \cdot k_{b}\left(a \in \mathrm{H} \backslash\left\{h_{\rho(s)}\right\}, b\right.$ belongs to a higher-weight chain or an element in $\{1, \ldots, r-1\})$ is expanded as a sum of chains defined by the refined graphic rule. Each in the sum has the general form

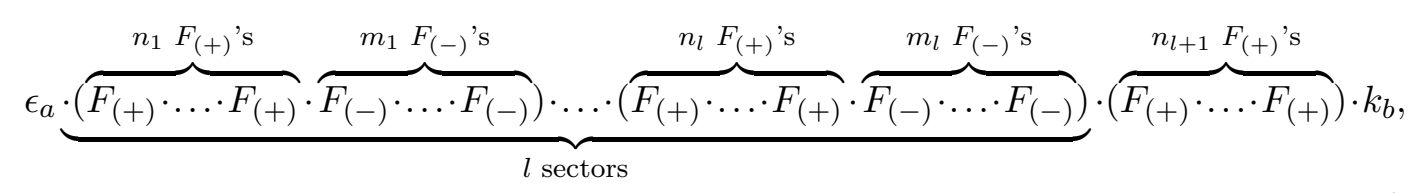

where $F_{(+)}^{\mu \nu} \equiv k^{\mu} \epsilon^{\nu}, F_{(-)}^{\mu \nu} \equiv-\epsilon^{\mu} k^{\nu}$ correspond to the nodes figure 1 (a) and (b), $l \geq 0$. In the language of graphs, such a chain is characterized by figure 9 (a). If $l=0, n_{l+1=1} \geq 0$, the chain eq. (5.1) in this case has no type-3 line. If $l>0$, the value of integers $n_{i}, m_{i}$ satisfy $n_{1}, n_{l+1} \geq 0, n_{i=2, \ldots, l}>0, m_{i=1, \ldots, l}>0$ and the chain in this case has at least one type-3 line.

(ii) Similarly, the chain $k_{h_{\rho(s)}} \cdot F_{i_{j}} \cdot \ldots \cdot F_{i_{1}} \cdot k_{b}(b \in\{1, \ldots, r-1\})$ can also be expanded as a sum of chains defined by the refined graphic rule. Each chain in the sum has the general form

$$
\begin{aligned}
k_{h_{\rho(s)}} & \cdot(\overbrace{F_{(-)} \cdot \ldots \cdot F_{(-)}}^{n_{0} F_{(-)} \text {'s }}) \cdot \underbrace{\overbrace{F_{(+)} \cdot \ldots \cdot F_{(+)}}^{n_{1} F_{(+)}} \cdot \overbrace{F_{(-)} \cdot \ldots \cdot F_{(-)}}^{m_{1}}) \cdot \ldots \cdot(\overbrace{F_{(+)} \cdot \ldots \cdot F_{(+)}}^{m_{l}} \cdot \overbrace{\left.F_{(-)} \cdot \ldots \cdot F_{(-)}\right)}^{n_{(-)} F_{(+)}})}_{l \text { sectors }} \\
& \cdot(\overbrace{F_{(+)} \cdot \ldots \cdot F_{(+)}}^{n_{l+1} F_{(+)} F_{(-)}}) \cdot k_{b},
\end{aligned}
$$

where $l \geq 0$. The value of integers $n_{i}, m_{i}$ satisfy $n_{0}, n_{l+1} \geq 0$ (for $l \geq 0$ ), $n_{i=1, \ldots, l}>0$, $m_{i=1, \ldots, l}>0$ (for $l>0$ ). In the language of graphs, this chain is characterized by figure 9 (b). Since the arrow lines connected to the starting node $h_{\rho(s)}$ and the ending node $b$ in figure 9 point to opposite directions, there must be at least one type- 3 line in figure 9 (b).

In order to obtain the skeleton $\mathcal{F}^{\prime}$ of a graph $\mathcal{F}$, we delete all type-3 lines from $\mathcal{F}$. Then both types of chains are divided into disjoint sectors in general. All sectors can be classified into the following types.

- Type-I sector: A sector containing a type-1 line (see figure 10 (a)). This sector can also have type- 2 lines whose arrows point to the direction of the two end nodes of the type- 1 line. Both types of chains figure 9 (a) and (b) can contain type-I sectors. 


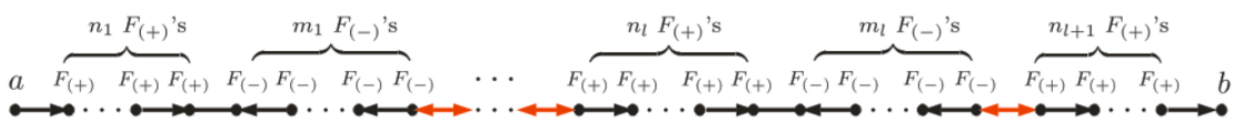

(a)

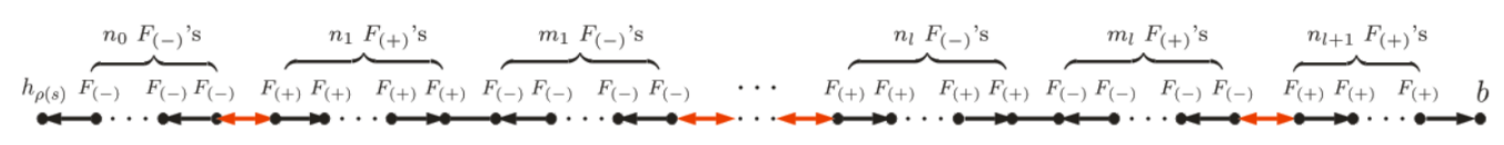

(b)

Figure 9. Graphs (a) and (b) correspond to chains led by $a \in \mathbf{H} \backslash\left\{h_{\rho(s)}\right\}$ and $h_{\rho(s)}$ defined by the refined graphic rule. A contraction of the form $\left(\ldots F_{(+)}\right) \cdot\left(F_{(-)} \cdot \ldots\right)$ (in (a) and (b)) or $\epsilon_{a} \cdot\left(F_{(-)} \cdot \ldots\right)$ (in (a)) provides a type-1 line, while a contraction of the form $\left(\ldots \cdot F_{(-)}\right) \cdot\left(F_{(+)} \cdot \ldots\right)$ (in (a) and (b)), $k_{h_{\rho(s)}} \cdot F_{(+)}$(in (b)), $F_{(-)} \cdot k_{b}$ (in (a) and (b)) or $k_{h_{\rho(s)}} \cdot k_{b}($ in (b)) provides a type-3 line.

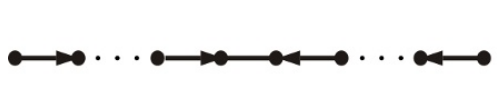

Type-I sector

(a)

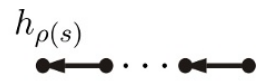

Type-II sector

(b)

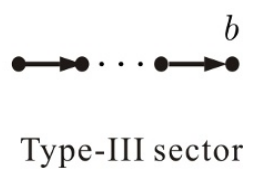

(c)

Figure 10. Three types of sectors of chains when all type-3 lines are removed.

- Type-II sector: A sector only containing type-2 lines whose arrows point to the direction of the starting node of the chain (see figure $10(\mathrm{~b})$ ). Only the highest-weight chain figure 9 (b) involve a type-II sector. The single node $h_{\rho(s)}$ with no line (on this chain) connected to it (i.e. figure 9 (b) with $n_{0}=0$ ) is considered as a special type-II sector.

- Type-III sector: A sector only containing type-2 lines whose arrows point to the direction of the ending node of a chain (see figure 10 (c)). Both types of chains figure 9 (a) and (b) contain type-III sectors. The single node $b$ with no line (on this chain) connected to it (i.e. figure 9 (a) or (b) with $n_{l+1}=0$ ) is a special type-III sector.

According to the refined graphic rule, the highest-weight chain in a graph must have form figure 9 (b). This chain at least contains two mutually disjoint sectors: a type-II sector and a type-III sector. It can also have type-I sectors. The type-III sector of the highestweight chain figure 9 (b) must end at a root $b \in\{1, \ldots, r-1\}$ while all nodes on this chain can be ending nodes of type-III sectors of other chains. If we look at a chain of the form figure 9 (a), we find that the type-III sector of this chain can end at either a node of a higherweight chain or a root $b \in\{1, \ldots, r-1\}$, while any node on this chain can be the ending node of the type-III sector of a lower-weight chain. After putting all these sectors together, we conclude that a general skeleton is composed of the following types of components:

- Type-I component: component containing a type-1 line (see the $\mathscr{A}$ and $\mathscr{B}$ components in figure 11 for example). Such a component consists of a type-I sector with possible type-III sectors attached to it. Each component of this type must have only one 

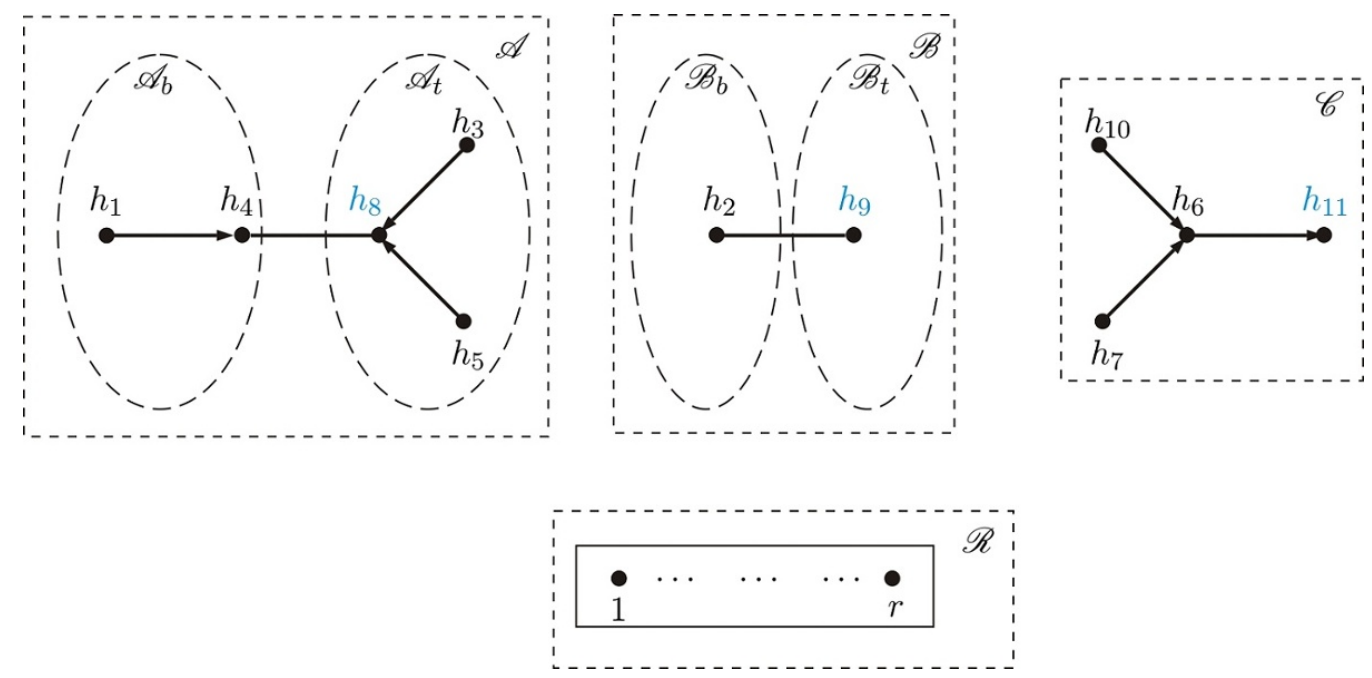

Figure 11. A skeleton consisting of four mutually disjoint components for the gauge invariance induced identity eq. (2.9) with $|\mathrm{H}|=11$. The reference order is chosen as $\mathrm{R}=\left\{h_{1}, h_{2}, \ldots, h_{10}, h_{11}\right\}$. Here, both $\mathscr{A}$ and $\mathscr{B}$ are type-I components. The kernel of $\mathscr{A}$ is given by the nodes $h_{4}$ and $h_{8}$ with a type-1 line between them (i.e. the factor $\epsilon_{h_{4}} \cdot \epsilon_{h_{8}}$ ), while the kernel of $\mathscr{B}$ is given by $h_{2}$ and $h_{9}$ with a type-1 line between them (i.e. $\epsilon_{h_{2}} \cdot \epsilon_{h_{9}}$ ). On each side of the component $\mathscr{A}$, the arrows of type-2 lines point to the corresponding end node of the kernel. The component $\mathscr{C}$ is the type-II component. The set $\mathscr{R}=\{1, \ldots, r\}$ itself is the type-III component (Although only the elements $1, \ldots, r-1$ can play as roots in the graphic rule, we also include the last element $r$ in the component $\mathscr{R}$ for convenience; a more general type-III componnet can involve type-II lines whose arrows pointing to roots).

type- 1 line in it and may also have type-2 lines pointing to the direction of the end nodes of the type- 1 line. A type-I component can be reconstructed by connecting a type- 1 line between two separate parts, each only contains type- 2 lines. We define the part to which the highest-weight node of a type-I component belongs as the top side. The other part is called the bottom side. The type- 1 line with its two end nodes together is defined as the kernel of this type-I component.

- Type-II component: the component containing the highest-weight graviton $h_{\rho(s)}$ (see the $\mathscr{C}$ components in figure 11 for example). This component consists of the type-II sector of the highest-weight chain with possible type-III sectors (belonging to other chains) attached on it. Apparently, type-II component involves only type-2 lines whose arrows point to the direction of the node $h_{\rho(s)}$.

- Type-III component: the component containing the set $\{1, \ldots, r\}$ (see the $\mathscr{R}$ components in figure 11 for example). This component is obtained by connecting possible type-III sectors to roots $b \in\{1, \ldots, r-1\}$. Thus type-III components contains type-2 lines whose arrows point to the root set.

All the above three types of components can be considered as connected subgraphs where tree structures with only type-2 lines pointing to (i) the kernel (for type-I components), (ii) the highest-weight node $h_{\rho(s)}$ (for the type-II component) and (iii) roots (for the type-III 
component). If the highest-weight chain contains only one type-3 line in the original graph $\mathcal{F}$ while other chains do not have any type-3 line, the skeleton of $\mathcal{F}$ must consist of only two mutually disjoint components: the type-II and the type-III components (see figure 6 (a)-(h) for example).

With the general structure of skeletons in hand, we will construct all graphs for an arbitrary skeleton in the next section.

\section{Constructing all physical and spurious graphs for a given skeleton}

As observed in section 4, all graphs corresponding to a given skeleton can be obtained by two steps: i) First, by attaching all type-I components to either the type-II or the type-III components via type-3 lines in a proper way, one constructs the final upper and lower blocks; ii) Second, for a given configuration of the final upper and lower blocks $\mathscr{U}, \mathscr{L}$, one obtains a (physical or spurious) graph by connecting arbitrary two nodes $a \in \mathscr{U}$ and $b \in \mathscr{L} \backslash\{r\}$ via a type-3 line. In this section, we generalize this observation to arbitrary case. We first show how to construct the final upper and lower blocks for a given skeleton. All physical and spurious graphs are then obtained by connecting a type-3 line between the final upper and lower blocks. We show that all spurious graphs must cancel in paires. In appendix $\mathrm{C}$ we further prove that all the physical graphs constructed by this section precisely match with the graphs directly obtained by refined graphic rule. With these discussions, we can rearrange the expression in the square brackets in eq. (3.5) s.t. it is expressed as a combination of so-called graph-based BCJ relations which will be proved as a combination of traditional BCJ relations.

\subsection{The construction of the final upper and lower blocks for a given skeleton}

Assuming that a skeleton is composed of $N+2$ components, as already analyzed in section 5 , there must be one type-II and one type-III component, which are respectively denoted by $\mathscr{C}^{\mathrm{II}}$ and $\mathscr{C}$ III. The number of type-I components is thus $N$. To construct the final upper and lower blocks, we should connect all $N$ type-I components $\mathscr{C}_{i}^{\mathrm{I}}$ towards the direction of either the type-II or the type-III component via $N$ type-3 lines so that the skeleton becomes a graph which only has two disjoint maximally connected subgraphs $\mathscr{U}$ and $\mathscr{L}$. More specifically, the final upper and lower blocks for a given skeleton is constructed as follows:

Step-1. Define the upper and lower blocks $\mathscr{U}$ and $\mathscr{L}$ by the type-II component $\mathscr{C}^{\mathrm{II}}$ (i.e. the component containing the highest weight node $\left.h_{\rho(h)}\right)$ and the type-III component $\mathscr{C}^{\text {III }}$ (i.e. the component containing elements $\{1, \ldots, r-1\})$ respectively. Define the reference order of all type-I components $\mathrm{R}_{\mathscr{C}}^{\mathrm{I}} \equiv\left\{\mathscr{C}_{1}^{\mathrm{I}}, \mathscr{C}_{2}^{\mathrm{I}}, \ldots, \mathscr{C}_{N}^{\mathrm{I}}\right\}$ by the relative order of their highestweight nodes in $\mathrm{R}=\left\{h_{\rho(1)}, h_{\rho(2)}, \ldots, h_{\rho(s)}\right\}$ which is already introduced in the (refined) graphic rule. We pick out the highest-weight type-I component $\mathscr{C}_{N}^{\mathrm{I}}$ as well as some other type-I components $\mathscr{C}_{a_{1}}^{\mathrm{I}}, \mathscr{C}_{a_{2}}^{\mathrm{I}}, \ldots, \mathscr{C}_{a_{i}}^{\mathrm{I}}$ (not necessary in the relative order in $\mathrm{R}_{\mathscr{C}}^{\mathrm{I}}$ ), then construct a chain of components which starts from $\mathscr{C}_{N}^{\mathrm{I}}$ and ends at either the upper block 
$\mathscr{U}$ or the lower block $\mathscr{L}^{5}$ Correspondingly, we get two possible structures

$$
\begin{aligned}
& \text { (i). } \mathscr{U} \rightarrow \mathscr{U}^{\prime}=\left[\left(\mathscr{C}_{N}^{\mathrm{I}}\right)_{t}-\left(\mathscr{C}_{N}^{\mathrm{I}}\right)_{b},\left(\mathscr{C}_{a_{i}}^{\mathrm{I}}\right)_{t(\text { or } b)}-\left(\mathscr{C}_{a_{i}}^{\mathrm{I}}\right)_{b(\text { or } t)}, \ldots,\left(\mathscr{C}_{a_{1}}^{\mathrm{I}}\right)_{t(\text { or } b)}-\left(\mathscr{C}_{a_{1}}^{\mathrm{I}}\right)_{b(\text { or } t)}, \mathscr{C}^{\mathrm{II}}\right] \text {, } \\
& \mathscr{L} \rightarrow \mathscr{L}^{\prime}=\mathscr{C}^{\mathrm{III}}
\end{aligned}
$$

(ii). $\mathscr{U} \rightarrow \mathscr{U}^{\prime}=\mathscr{C}^{\mathrm{II}}$,

$$
\mathscr{L} \rightarrow \mathscr{L}^{\prime}=\left[\left(\mathscr{C}_{N}^{\mathrm{I}}\right)_{t}-\left(\mathscr{C}_{N}^{\mathrm{I}}\right)_{b},\left(\mathscr{C}_{a_{i}}^{\mathrm{I}}\right)_{t(\text { or } b)}-\left(\mathscr{C}_{a_{i}}^{\mathrm{I}}\right)_{b(\text { or } t)}, \ldots,\left(\mathscr{C}_{a_{1}}^{\mathrm{I}}\right)_{t(\text { or } b)}-\left(\mathscr{C}_{a_{1}}^{\mathrm{I}}\right)_{b(\text { or } t)}, \mathscr{C}^{\mathrm{III}}\right] .
$$

Redefine $\mathrm{R}_{\mathscr{C}}^{\mathrm{I}}$ by $\mathrm{R}_{\mathscr{C}}^{\mathrm{I}}{ }^{\prime}=\mathrm{R}_{\mathscr{C}}^{\mathrm{I}} \backslash\left\{\mathscr{C}_{N}^{\mathrm{I}}, \mathscr{C}_{a_{1}}^{\mathrm{I}}, \ldots, \mathscr{C}_{a_{i}}^{\mathrm{I}}\right\}, \mathscr{U}$ and $\mathscr{L}$ by the new obtained upper and lower blocks $\mathscr{U}^{\prime}$ and $\mathscr{L}^{\prime}$ respectively.

Step-2. Pick out the highest-weight component $\mathscr{C}_{a_{i^{\prime}}^{\prime}}^{\mathrm{I}}$ and arbitrary components $\mathscr{C}_{a_{1}^{\prime}}^{\mathrm{I}}, \ldots$, $\mathscr{C}_{a_{i^{\prime}-1}^{\prime}}^{\mathrm{I}}$ from $\mathrm{R}_{\mathscr{C}}^{\mathrm{I}}{ }^{\prime}$ defined in the previous step. Construct a chain, whose starting component is $\mathscr{C}_{a_{i^{\prime}}^{\prime}}^{\mathrm{I}}$ and internal components are $\mathscr{C}_{a_{1}^{\prime}}^{\mathrm{I}}, \ldots, \mathscr{C}_{a_{i^{\prime}-1}^{\prime}}^{\mathrm{I}}$, towards either the upper block $\mathscr{U}^{\prime}$ or the lower one $\mathscr{L}^{\prime}$ :

$$
\begin{aligned}
&(i) . \mathscr{U} \rightarrow \mathscr{U}^{\prime \prime}=\left[\left(\mathscr{C}_{a_{i^{\prime}}^{\prime}}^{\mathrm{I}}\right)_{t}-\left(\mathscr{C}_{a_{i^{\prime}}^{\prime}}^{\mathrm{I}}\right)_{b},\left(\mathscr{C}_{a_{i^{\prime}-1}^{\prime}}^{\mathrm{I}}\right)_{t(\text { or } b)}-\left(\mathscr{C}_{a_{i^{\prime}-1}^{\prime}}^{\mathrm{I}}\right)_{b(\text { or } t)},\right. \\
&\left.\ldots,\left(\mathscr{C}_{a_{1}^{\prime}}^{\mathrm{I}}\right)_{t(\text { or } b)}-\left(\mathscr{C}_{a_{1}^{\prime}}^{\mathrm{I}}\right)_{b(\mathrm{or} t)}, \mathscr{U}^{\prime}\right], \\
& \mathscr{L} \rightarrow \mathscr{L}^{\prime \prime}=\mathscr{L}^{\prime} ; \\
&(i i) . \mathscr{U} \rightarrow \mathscr{U}^{\prime \prime}=\mathscr{U}^{\prime}, \\
& \mathscr{L} \rightarrow \mathscr{L}^{\prime \prime}=\left[\left(\mathscr{C}_{a_{i^{\prime}}^{\prime}}^{\mathrm{I}}\right)_{t}-\left(\mathscr{C}_{a_{i^{\prime}}^{\prime}}^{\mathrm{I}}\right)_{b},\left(\mathscr{C}_{a_{i^{\prime}-1}^{\prime}}^{\mathrm{I}}\right)_{t(\text { or } b)}-\left(\mathscr{C}_{a_{i^{\prime}-1^{\prime}}}^{\mathrm{I}}\right)_{b(\text { or } t)},\right. \\
&\left.\ldots,\left(\mathscr{C}_{a_{1}^{\prime}}^{\mathrm{I}}\right)_{t(\text { or } b)}-\left(\mathscr{C}_{a_{1}^{\prime}}^{\mathrm{I}}\right)_{b(\text { or } t)}, \mathscr{L}^{\prime}\right] .
\end{aligned}
$$

Again, redefine $\mathrm{R}_{\mathscr{C}}$ by $\mathrm{R}_{\mathscr{C}}^{\prime \prime}=\mathrm{R}_{\mathscr{C}}^{\prime} \backslash\left\{\mathscr{C}_{a_{1}^{\prime}}^{\mathrm{I}}, \ldots, \mathscr{C}_{a_{i^{\prime}}^{\prime}}^{\mathrm{I}}\right\}$.

Step-3. Repeat the above steps until the ordered set $\mathrm{R}_{\mathscr{C}}$ becomes empty. In each step, we construct a chain which is led by the highest-weight component in the redefined $\mathrm{R}_{\mathscr{C}}$, towards either the upper block $\mathscr{U}$ or the lower block $\mathscr{L}$ obtained in the previous step. Then redefine the upper and lower blocks by the new constructed maximally connected subgraphs that containing the highest-weight node $h_{\rho(s)}$ and roots $\{1, \ldots, r-1\}$, respectively.

After the above steps, graphs with only two disjoint connected blocks (the final upper and lower blocks $\mathscr{U}$ and $\mathscr{L}$ ) are finally produced. A simple example is already given by figure 8 . A more complicated example is given by the construction of all possible configurations of the final upper and lower blocks for the skeleton figure 11 and can be

\footnotetext{
${ }^{5}$ Picking out nodes $x_{i+1} \in\left(\mathscr{C}_{N}^{\mathrm{I}}\right)_{b}, y_{j} \in\left(\mathscr{C}_{a_{j}}^{\mathrm{I}}\right)_{t \text { or } b}(j=1, \ldots i), x_{j} \in\left(\mathscr{C}_{a_{j}}^{\mathrm{I}}\right)_{b \text { or } t}(j=1, \ldots, i)$ and $y_{0} \in \mathscr{C}^{\mathrm{II}}$, a chain of components, which starts from $\mathscr{C}_{N}^{\mathrm{I}}$, passes through $\mathscr{C}_{a_{1}}^{\mathrm{I}}, \mathscr{C}_{a_{2}}^{\mathrm{I}}, \ldots, \mathscr{C}_{a_{i}}^{\mathrm{I}}$ and ends at $\mathscr{C}^{\mathrm{II}}$, is defined by connecting each $\left(x_{j+1}, y_{j}\right)$ pair (for $j=i, i-1, \ldots, 1$ ) via a type-3 line and denoted by $\left[\left(\mathscr{C}_{N}^{\mathrm{I}}\right)_{t}-\left(\mathscr{C}_{N}^{\mathrm{I}}\right)_{b},\left(\mathscr{C}_{a_{i}}^{\mathrm{I}}\right)_{t(\text { or } b)}-\left(\mathscr{C}_{a_{i}}^{\mathrm{I}}\right)_{b(\text { or } t)}, \ldots,\left(\mathscr{C}_{a_{1}}^{\mathrm{I}}\right)_{t(\text { or } b)}-\left(\mathscr{C}_{a_{1}}^{\mathrm{I}}\right)_{b(\text { or } t)}, \mathscr{C}^{\mathrm{II}}\right]$. There are two important features for chains of components: (1) All the kernels of the starting and internal components must be passed through by this chain (i.e. the two nodes $y_{j}, x_{j}$ for a given $j=1, \ldots, i$ must coming from opposite sides of the component $\mathscr{C}_{a_{j}}^{\mathrm{I}}$ ). (2) A chain must starts from the top side of the starting component (i.e. $x_{i+1}$ must belong to the bottom side of $\mathscr{C}_{N}^{\mathrm{I}}$ ).
} 
found in appendix $\mathrm{C}$ (the graphs obtained from figure 29 (a1), (b1), (c1), (d1), figure 30 (a1), (b1), (c1), (d1) and figure 31 (a1), (b1), (c1), (d1) by removing all dashed red lines).

\subsection{Physical and spurious graphs for a given configuration of the final upper and lower blocks}

For a given skeleton $\mathcal{F}^{\prime}$ and a given configuration of the final upper and lower blocks $\mathscr{U}$ and $\mathscr{L}$, a graph is obtained by connecting two arbitrary nodes respectively belonging to $\mathscr{U}$ and $\mathscr{L} \backslash\{r\}$ via a type-3 line. In general, such a graph may not be allowed by the refined graphic rule (e.g. the spurious graphs figure 7 (c) and (f)). Therefore, we should analyze whether such a graph is a physical or a spurious one. This can be achieved by noting the following constraints from the refined graphic rule:

- Assume we have two components $\mathscr{C}^{\mathrm{I}}$ and $\mathscr{C}$. Here, $\mathscr{C}^{\mathrm{I}}$ is a type-I component while $\mathscr{C}$ is either the type-II or a type-I component whose weight is higher than that of $\mathscr{C}^{\mathrm{I}}$. The structures shown by figure 12 (a) and (b), ${ }^{6}$ in which the chain (of nodes) led by the highest weight node in $\mathscr{C}$ passes through only a single side (the top or the bottom side) of the component $\mathscr{C}^{\mathrm{I}}$ (via two type-3 lines), are forbidden. This is because if only the top (or bottom) side of $\mathscr{C}^{\mathrm{I}}$ is passed through by the chain, there must exist an internal node which is attached by two conflict arrows on that chain (for example the node $h_{8}$ in figure $12(\mathrm{c})$ ). Such a structure is forbidden by the definition of the strength tensor $F^{\mu \nu}$. Therefore, only the graphs figure 12 (d) and (e) where the higher-weight chain pass through both sides of $\mathscr{C}^{\mathrm{I}}$ are allowed (a more specific example is given by figure 12 (f)). In other words, if a chain of higher weight passes through a type-I component $\mathscr{C}^{\mathrm{I}}$, the kernel of the component $\mathscr{C}^{\mathrm{I}}$ (e.g. $\epsilon_{h_{4}} \cdot \epsilon_{h_{8}}$ for figure 12 (f)) must be on this chain.

- A chain, say $\mathbb{C H}_{1}$, starting at the highest-weight node of a type-I component $\mathscr{C}^{\mathrm{I}}$ must pass through both sides of $\mathscr{C}^{\mathrm{I}}$ (or equivalently the kernel of $\mathscr{C}^{\mathrm{I}}$ ). If not (as shown by figure 13 (a) or a more explicit example figure 13 (c)), there must exist another chain, say $\mathbb{C H}_{2}$ which ends at some node $a \in \mathscr{C}_{t}^{\mathrm{I}} \cap \mathbb{C H}_{1}$. The node $a$ plays as the ending node of $\mathbb{C H}_{2}$ but can only supply a $\epsilon$ to $\mathbb{C H}_{2}$, which apparently conflicts with the refined graphic rule.

Recalling that a chain of components (which just reflects the chain lead by the highest weight node in these components) defined in section 6.1 always starts at the top side of its starting component, the structure shown by figure 13 (a) is automatically avoided when the final upper and lower blocks are connected by a type-3 line. Hence only the nonphysical structures figure 12 (a), (b) may appear. If a graph obtained by connecting the final upper and lower blocks via a type-3 line does not contain any structure of figure 12 (a) and (b), it is a physical one; otherwise, spurious. As an example, all physical graphs for the skeleton

\footnotetext{
${ }^{6}$ We use a circle to stand for an arbitrary component or the top or bottom side of a type-I component; a solid line between the top and bottom sides of a type-I component $\mathscr{C}^{\mathrm{I}}$ stands for a type-1 line connecting two arbitrary nodes on the corresponding sides; a double arrow line between two distinct components stands for a type-3 line between two arbitrary nodes in corresponding components.
} 


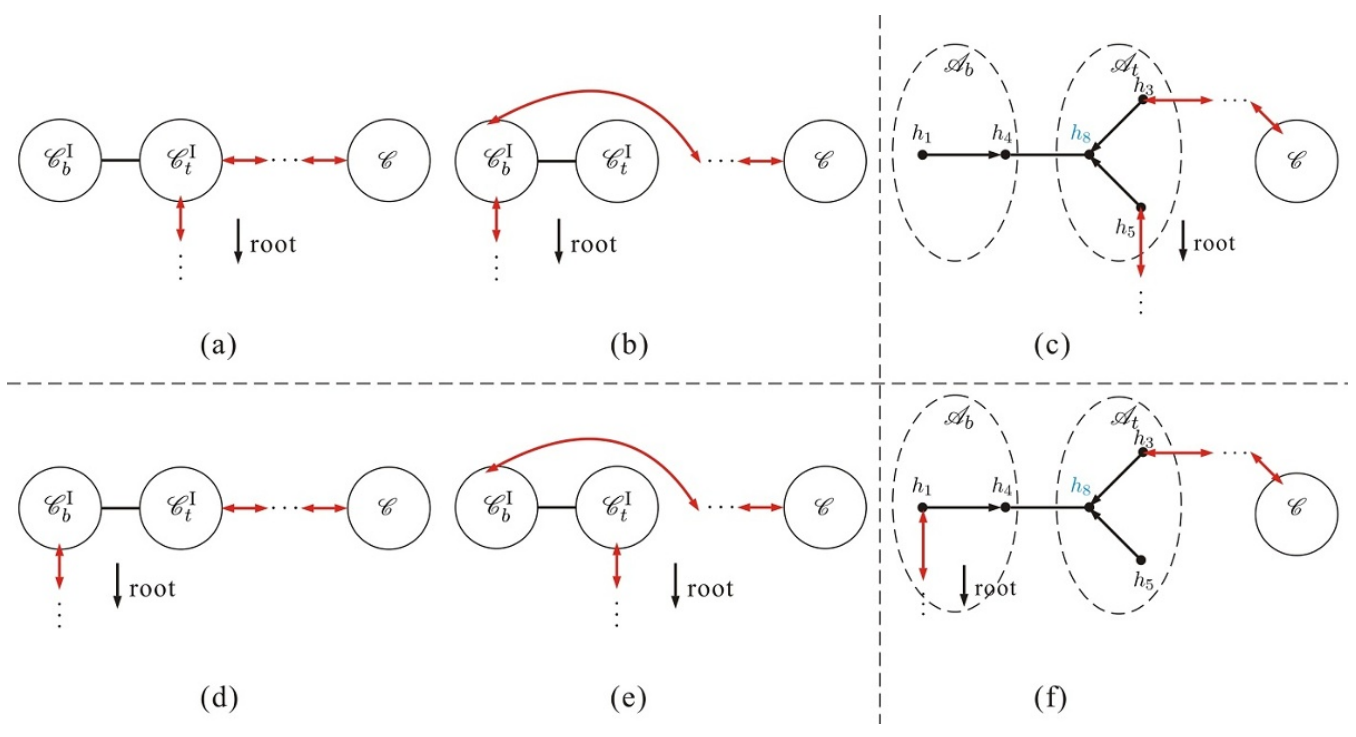

Figure 12. Assume that $\mathscr{C}$ is the type-II component or a type-I component with a higher weight than $\mathscr{C}^{\mathrm{I}}$. The graphs (a) and (b), in which a higher-weight chain passes through only one side of $\mathscr{C}^{\mathrm{I}}$, are not allowed by the refined graphic rule because a chain cannot contain conflict arrow line towards root (e.g. the graph (c)). Thus if a chain passes through a component, it must pass through both sides of the component. In other words, the kernel of the component $\mathscr{C}^{\mathrm{I}}$ must be on that chain (see the graphs (d) and (e) as well as the explicit example (f)).

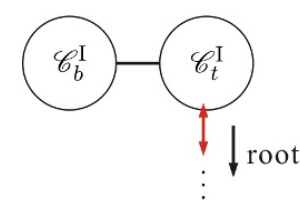

(a)

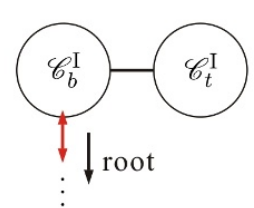

(b)

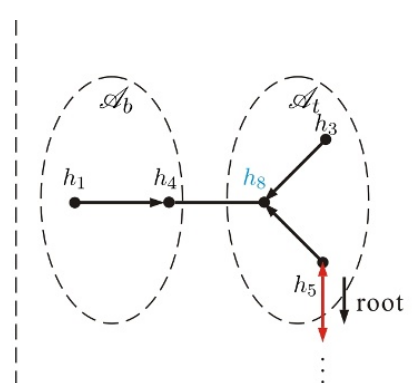

(c)

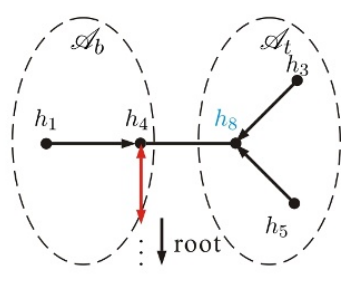

(d)

Figure 13. If there is a chain starting at the highest-weight node of a type-I component $\mathscr{C}^{\mathrm{I}}$, this chain must contain the kernel of $\mathscr{C}^{\mathrm{I}}$. In other words, the bottom-side end node of the kernel must be nearer to root than the top-side one. Thus the graph (b) (a more concrete example is given by (d)) is allowed but (a) (a more concrete example is given by (c)) is not allowed.

figure 11 are given by figure 29 (a1), (b1), (c1), (d1), figure 30 (a1), (b1), (c1), (d1) and figure 31 (a1), (b1), (c1), (d1), while all spurious graphs are given by figure 29 (a2), (b2), (c2), (d2), figure 30 (a2), (b2), (c2), (d2) and figure 31 (a2), (b2), (c2), (d2). The (physical and spurious) graphs on each row of figure 29, figure 30 and figure 31 correspond to a same configuration of the final upper and lower blocks.

In appendix D, we prove that all the physical graphs corresponding to all possible configurations of the final upper and lower blocks for a skeleton $\mathcal{F}^{\prime}$ precisely reproduce all $\mathscr{F} \supset \mathscr{F}^{\prime}$ (in eq. (3.5)) defined by the refined graphic rule. In the remaining of this 


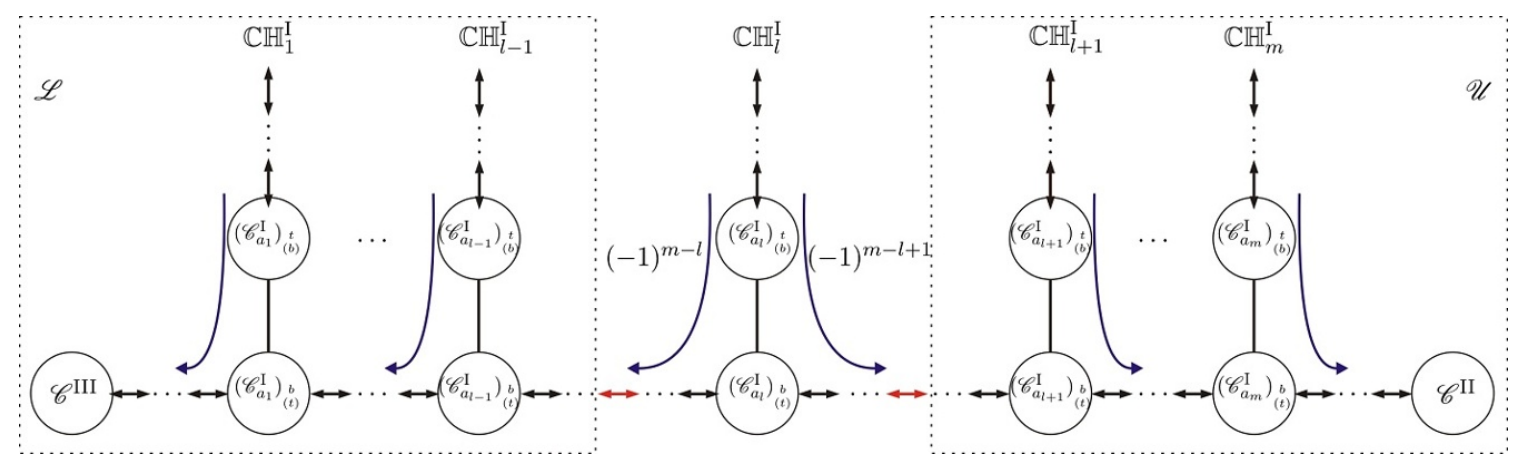

Figure 14. A typical spurious graph. There are $m$ spurious structures $\mathscr{C}_{a_{2}}^{\mathrm{I}}, \ldots, \mathscr{C}_{a_{m}}^{\mathrm{I}}$ on the path from the highest weight node $h_{\rho(s)}$ towards a root. The chains $\mathbb{C} \mathbb{H}_{1}^{\mathrm{I}}, \mathbb{C} \mathbb{H}_{2}^{\mathrm{I}}, \ldots, \mathbb{C} \mathbb{H}_{l-1}^{\mathrm{I}}\left(\mathbb{C} \mathbb{H}_{l+1}^{\mathrm{I}}\right.$, $\left.\mathbb{C H} \mathbb{H}_{l+2}^{\mathrm{I}}, \ldots, \mathbb{C H}_{m}^{\mathrm{I}}\right)$ containing $\mathscr{C}_{a_{1}}^{\mathrm{I}}, \mathscr{C}_{a_{2}}^{\mathrm{I}}, \ldots, \mathscr{C}_{a_{l-1}}^{\mathrm{I}}\left(\mathscr{C}_{a_{l+1}}^{\mathrm{I}}, \mathscr{C}_{a_{l+2}}^{\mathrm{I}}, \ldots, \mathscr{C}_{a_{m}}^{\mathrm{I}}\right)$ must belong to the final lower (upper) block. The lowest-weight chain $\mathbb{C} \mathbb{H}_{l}^{\mathrm{I}}$ among $\mathbb{C} \mathbb{H}_{i}^{\mathrm{I}}(i=1, \ldots, m)$ can belong to either the final upper block (with sign $(-1)^{m-l+1}$ ) or the final lower one (with a sign $(-1)^{m-l}$ ). Thus two spurious graphs with the same configuration but opposite signs are obtained from two distinct configurations of the final upper and lower blocks, respectively.

subsection, we associate spurious graphs with signs properly, s.t. all these spurious graphs for a given skeleton cancel out.

\section{Signs of spurious graphs}

Spurious graphs may appear when we connect the final upper and lower blocks into a connected graph by a type- 3 line. This is because the chain led by the highest-weight node $h_{\rho(s)} \in \mathscr{C}^{\mathrm{II}}$ is produced and may have the structures figure 12 (a), (b) on it. We can find the unique path starting from $h_{\rho(s)} \in \mathscr{C}^{\mathrm{II}}$ and ending at a root in $\{1, \ldots, r-1\}$ (thus in $\left.\mathscr{C}^{\mathrm{III}}\right)$. If there exist $m(m \geq 1)$ type-I components $\mathscr{C}_{a_{1}}^{\mathrm{I}}, \mathscr{C}_{a_{2}}^{\mathrm{I}}, \ldots, \mathscr{C}_{a_{m}}^{\mathrm{I}}$ s. t. only a single side of each is on the path, as shown by figure 12 (a), (b), the graph must be a spurious one. Such a component $\mathscr{C}_{a_{i}}^{\mathrm{I}}$ is called a spurious component. The sign associated to the spurious graph is defined by $(-1)^{\mathcal{S}(\mathscr{U})}$, where $\mathcal{S}(\mathscr{U})$ is the number of spurious components in the final upper block. For example, in figure $30(\mathrm{c} 2), \mathcal{S}(\mathscr{U})=1,2,1$ for the graphs with lines $5-, 12+, 15-$ correspondingly.

To show that spurious graphs for distinct configurations always cancel in pairs, we assume that the path from $h_{\rho(s)} \in \mathscr{C}^{\mathrm{II}}$ towards a root passes through spurious components $\mathscr{C}_{a_{i}}^{\mathrm{I}}(i=1, \ldots, m)$ in the order $\mathscr{C}_{a_{m}}^{\mathrm{I}}, \ldots, \mathscr{C}_{a_{2}}^{\mathrm{I}}, \mathscr{C}_{a_{1}}^{\mathrm{I}}$ for convenience. The chains containing components $\mathscr{C}_{a_{1}}^{\mathrm{I}}, \mathscr{C}_{a_{2}}^{\mathrm{I}}, \ldots, \mathscr{C}_{a_{m}}^{\mathrm{I}}$ are correspondingly denoted by $\mathbb{C} \mathbb{H}_{1}^{\mathrm{I}}, \mathbb{C} \mathbb{H}_{2}^{\mathrm{I}}, \ldots, \mathbb{C H}_{m}^{\mathrm{I}}$ and their weights are denoted by $\mathcal{W}_{1}^{\mathrm{I}}, \mathcal{W}_{2}^{\mathrm{I}}, \ldots, \mathcal{W}_{m}^{\mathrm{I}}$. The lowest-weight chain among $\mathbb{C} \mathbb{H}_{1}^{\mathrm{I}}, \mathbb{C H}_{2}^{\mathrm{I}}$, $\ldots, \mathbb{C} \mathbb{H}_{m}^{\mathrm{I}}$ is assumed to be $\mathbb{C} \mathbb{H}_{l}^{\mathrm{I}}$. This typical spurious graph is shown by figure 14 . We find that the following properties of chains $\mathbb{C H}_{1}^{\mathrm{I}}, \mathbb{C} \mathbb{H}_{2}^{\mathrm{I}}, \ldots, \mathbb{C} \mathbb{H}_{m}^{\mathrm{I}}$ in a given spurious graph must be satisfied:

(i) The weights of chains $\mathcal{W}_{1}^{\mathrm{I}}, \mathcal{W}_{2}^{\mathrm{I}}, \ldots, \mathcal{W}_{m}^{\mathrm{I}}$ must satisfy

$$
\mathcal{W}_{1}^{\mathrm{I}}>\mathcal{W}_{2}^{\mathrm{I}}>\cdots>\mathcal{W}_{l-1}^{\mathrm{I}}>\mathcal{W}_{l}^{\mathrm{I}}, \quad \mathcal{W}_{l}^{\mathrm{I}}<\mathcal{W}_{l+1}^{\mathrm{I}}<\cdots<\mathcal{W}_{m-1}^{\mathrm{I}}<\mathcal{W}_{m}^{\mathrm{I}}
$$


This is because we always connect chains, in the descending order of their weights, to either the upper or the lower block (as shown in section 6.1).

(ii) The chains $\mathbb{C H} \mathbb{H}_{1}^{\mathrm{I}}, \mathbb{C} \mathbb{H}_{2}^{\mathrm{I}}, \ldots, \mathbb{C} \mathbb{H}_{l-1}^{\mathrm{I}}\left(\mathbb{C H}_{l+1}^{\mathrm{I}}, \mathbb{C H}_{l+2}^{\mathrm{I}}, \ldots, \mathbb{C} \mathbb{H}_{m}^{\mathrm{I}}\right)$ and structures attached to them belong to the final lower (upper) block (see figure 14). If not, for example the chain $\mathbb{C H}_{i}^{\mathrm{I}}$ for given $i<l$ belongs to the final upper block, there must be at least one component (i.e. $\mathscr{C}_{a_{l}}^{\mathrm{I}}$ ) with only a single side on $\mathbb{C H}_{i}^{\mathrm{I}}$ before connecting the upper and lower blocks together. This conflicts with the construction in section 6.1).

According to the construction process in section 6.1 , the lowest-weight chain $\mathbb{C} \mathbb{H}_{l}^{\mathrm{I}}$ (and structure attached to it) among $\mathbb{C H} \mathbb{H}_{1}^{\mathrm{I}}, \mathbb{C H}_{2}^{\mathrm{I}}, \ldots, \mathbb{C} \mathbb{H}_{m}^{\mathrm{I}}$ can be considered to be connected with either the upper or the lower block, which have been defined in the previous steps, via a type-3 line (see figure 14). Thus the chain $\mathbb{C H}_{l}^{\mathrm{I}}$ (and structure attached to it) in a given spurious graph can belong to either the final upper block or the final lower block. Correspondingly, the signs for these two spurious graphs, which have the same structure and distinct configurations of the final upper and lower blocks, are $(-1)^{m-l+1}$ and $(-1)^{m-l}$. As a result, all the spurious graphs for a given skeleton must cancel out in pairs.

\subsection{The sum over all physical and spurious graphs}

Now we are ready to rearrange the expression in the square brackets of eq. (3.5) in an appropriate form for studying the relationship between the gauge invariance induced identity eq. (2.9) and BCJ relations. For any skeleton $\mathcal{F}^{\prime}$, the expression in the square brackets of eq. (3.5) is given by

$$
I=\sum_{\substack{\mathcal{F} \text { s.t. } \\ \mathcal{F} \supset \mathcal{F}^{\prime}}} \mathcal{K}^{\left[\mathcal{F} \backslash \mathcal{F}^{\prime}\right]} \sum_{\sigma^{\mathcal{F}}} A\left(1, \boldsymbol{\sigma}^{\mathcal{F}}, r\right) .
$$

The sum over all (physical) graphs $\mathcal{F}$ containing the skeleton $\mathcal{F}^{\prime}$ can be achieved by the following two summations:

(i) Sum over all possible configurations $\mathscr{U} \oplus \mathscr{L},{ }^{7}$ of the final upper and lower blocks $\mathscr{U}$ and $\mathscr{L}$ constructed in section 6.1, including (1) all possible configurations of $\mathscr{U}$ and $\mathscr{L}$ constructed by chains of components when neglecting the inner structures of all type-II, type-III components and both sides of type-I components; (2) all possible choices of end nodes of the type-3 lines for each configuration in (1). The kinematic factor $\mathcal{K}^{\left[\mathscr{U} \oplus \mathscr{L} \backslash \mathcal{F}^{\prime}\right]}$ with respect to a given configuration $\mathscr{U} \oplus \mathscr{L}$ is the product of $k \cdot k$ factors corresponding to the type-3 lines in the final upper and lower blocks $\mathscr{U}, \mathscr{L}$.

(ii) For a given configuration $\mathscr{U} \oplus \mathscr{L}$ in the previous summation, sum over all possible physical and spurious graphs $\mathcal{F} \in(\mathscr{U}, \mathscr{L})$ which are obtained by connecting two nodes in distinct blocks via a type-3 line (recalling that the sum of all spurious graphs cancel in pairs). The kinematic factor provided in this step is the $k \cdot k$ factor for the type-3 line between the two blocks and is denoted by $\mathcal{K}^{[\mathcal{F} \backslash \mathscr{U} \oplus \mathscr{L}]}$.

\footnotetext{
${ }^{7}$ Here, $\oplus$ denotes the disjoint union.
} 
To sum up, $I$ can be expressed by

$$
I=\sum_{\mathscr{U} \oplus \mathscr{L}} \mathcal{K}^{\left[\mathscr{U} \oplus \mathscr{L} \backslash \mathcal{F}^{\prime}\right]} I[\mathscr{U}, \mathscr{L}] .
$$

The $I[\mathscr{U}, \mathscr{L}]$ for a given configuration $\mathscr{U} \oplus \mathscr{L}$ in the above equation is defined by

$$
I[\mathscr{U}, \mathscr{L}] \equiv \sum_{\mathcal{F} \in(\mathscr{U}, \mathscr{L})}(-)^{\mathcal{F}} \mathcal{K}^{[\mathcal{F} \backslash \mathscr{U} \oplus \mathscr{L}]} \sum_{\boldsymbol{\sigma}^{\mathcal{F}}} A\left(1, \boldsymbol{\sigma}^{\mathcal{F}}, r\right)
$$

where

$$
(-)^{\mathcal{F}}=(-1)^{\mathcal{N}(\mathscr{U})+\mathcal{N}(\mathscr{L})+\mathcal{S}(\mathscr{U})+1}
$$

is a proper sign depending on the total number of arrows pointing away from the direction of root $(\mathcal{N}(\mathscr{U})$ for the final upper block, $\mathcal{N}(\mathscr{L})$ for the final lower block and 1 for the type-3 line between the two blocks) and the number $\mathcal{S}(\mathscr{U})$ of spurious components in the final upper block of a graph.

\section{Comments on the expression eq. (6.6)}

Permutations $\boldsymbol{\sigma}^{\mathcal{F}}$. From the graphic rule, we can see permutations $\boldsymbol{\sigma}^{\mathcal{F}}$ for a given graph $\mathcal{F} \in(\mathscr{U}, \mathscr{L})$ do not rely on types of lines. They only depend on the relative positions between nodes in a graph. Therefore, in the following we replace all types of lines in $\mathcal{F}$ by dashed lines with no arrow when considering permutations $\boldsymbol{\sigma}^{\mathcal{F}}$ established by a graph $\mathcal{F}$. For each graph $\mathcal{F}$, the possible permutations $\boldsymbol{\sigma}^{\mathcal{F}}$ are collected as follows:

- The relative order of roots is always $\left(\sigma^{\mathcal{F}}\right)^{-1}(1)<\left(\sigma^{\mathcal{F}}\right)^{-1}(2)<\cdots<\left(\sigma^{\mathcal{F}}\right)^{-1}(r-1)$. We set $\sigma^{-1}(r)=\infty$ to require that the element $r$ is always the last one in the permutation $\boldsymbol{\sigma}^{\mathcal{F}}$.

- For tree structures planted at roots $\{1,2, \ldots, r-1\}$, if two elements $a, b \in \mathbf{H}$ are on a same path which starts from an element of $\mathrm{H}$ and end at an arbitrary root $l \in\{1,2, \ldots, r-1\}$ and $a$ is nearer to the root $l$ than $b$, we have $\left(\sigma^{\mathcal{F}}\right)^{-1}(l)<$ $\left(\sigma^{\mathcal{F}}\right)^{-1}(a)<\left(\sigma^{\mathcal{F}}\right)^{-1}(b)$.

- If a node $a \in \mathrm{H}$ in a connected tree structure $\mathcal{T}$ (shown by figure 15 (a)) is nearer to the root than all other nodes in $\mathcal{T}$ and $a$ is attached to by $M$ connected sub-tree structures (branches) $\mathcal{T}_{1}, \mathcal{T}_{2}, \ldots, \mathcal{T}_{M}(M \geq 2$ ) (where the nodes nearest to $a$ in these branches are $a_{1}, a_{2}, \ldots, a_{M}$ correspondingly), the collection of all possible relative orders for nodes in $\mathcal{T}$ are recursively expressed by

$$
\left.\mathcal{T}\right|_{a}=\left\{a,\left.\left.\left.\mathcal{T}_{1}\right|_{a_{1}} \uplus \mathcal{T}_{2}\right|_{a_{2}} \uplus \cdots \uplus \mathcal{T}_{M}\right|_{a_{M}}\right\},
$$

where $\left.\mathcal{T}\right|_{a}$ denotes all possible permutations established by $\mathcal{T}$ with the leftmost element $a$. 


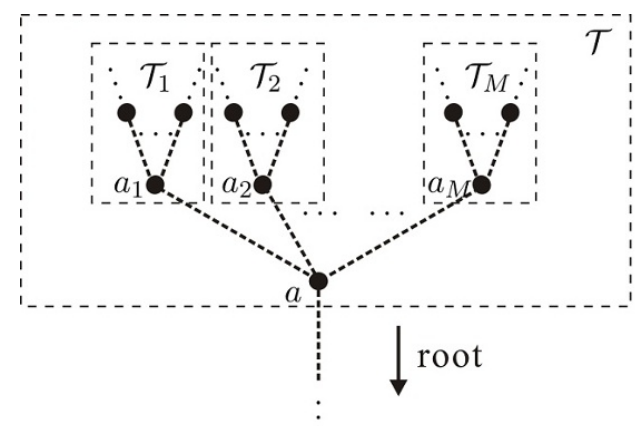

(a)

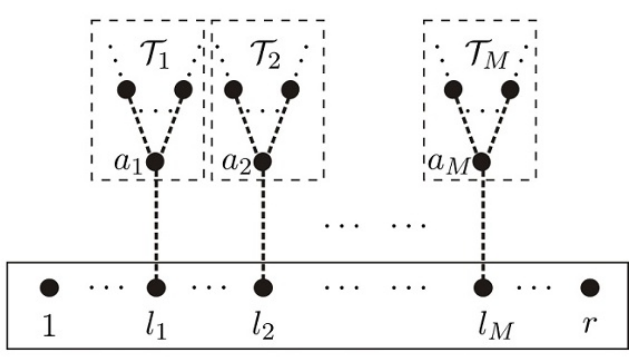

(b)

Figure 15. (a): a typical tree structure with sub-trees planted at the node $a,(\mathrm{~b})$ : a typical graph with trees planted at roots $l_{1}, \ldots, l_{M} \in\{1, \ldots, r-1\}$.

- Suppose there are $M$ connected tree structures $\mathcal{T}_{1}, \mathcal{T}_{2}, \ldots, \mathcal{T}_{M}$ planted at roots $l_{1}, l_{2}, \ldots, l_{M} \in\{1, \ldots, r-1\}$ in a graph $\mathcal{F}$ correspondingly while the nearest-toroots elements with respect to these tree structures are $a_{1}, a_{2}, \ldots, a_{M}$ (see figure 15 (b)). The permutations $\boldsymbol{\sigma}^{\mathcal{F}}$ of elements in $\mathrm{H} \cup\{1, \ldots, r\}$ satisfy

$$
\left.\boldsymbol{\sigma}^{\mathcal{F}} \in\left\{1,\left.\left.\left.\{2, \ldots, r-1\} ш \mathcal{T}_{1}\right|_{a_{1}} \uplus \mathcal{T}_{2}\right|_{a_{2}} \amalg \cdots ш \mathcal{T}_{M}\right|_{a_{M}}, r\right\}\right|_{\substack{\left.l_{i} \prec a_{i} \\ i=1, \ldots, M\right)}},
$$

where $a \prec b$ in a permutation $\sigma$ means $\sigma^{-1}(a)<\sigma^{-1}(b)$.

Rearrangement of eq. (6.6). Since the graph $\mathcal{F}$ is given by connecting any node $a$ in the final upper block $\mathscr{U}$ and any node $b$ in the final lower block $\mathscr{L}$ via a type-3 line, the factor $\mathcal{K}^{[\mathcal{F} \backslash \mathscr{U} \oplus \mathscr{L}]}$ is just $k_{a} \cdot k_{b}$ while the sum over all $\mathcal{F} \in(\mathscr{U}, \mathscr{L})$ is given by summing over all choices of nodes $a \in \mathscr{U}$ and $b \in \mathscr{L}$. Permutations $\sigma^{\mathcal{F}}$ can be understood as follows:

(i) Suppose that all tree structures planted at roots $l_{1}, l_{2}, \ldots, l_{M} \in\{1, \ldots, r-1\}$ in a given (physical or spurious) graph $\mathcal{F}$ are $\mathcal{T}_{1}, \mathcal{T}_{2}, \ldots, \mathcal{T}_{M}$ and the nearest-to-root elements with respect to these tree structures are $a_{1}, a_{2}, \ldots, a_{M}$ correspondingly (see figure 16). The final upper block $\mathscr{U}$ (connected to the node $b \in \mathscr{L}$ ), when all lines therein are replaced by dashed lines, must belong to some connected tree structure $\mathcal{T}_{i}$ which is attached to a root $l_{i}$. Permutations $\boldsymbol{\sigma}^{\mathcal{F}}$ then satisfy

$$
\left.\sigma^{\mathcal{F}} \in\left\{1,\left.\left.\left.\{2, \ldots, r-1\} ш \mathcal{T}_{1}\right|_{a_{1}} \uplus \cdots \uplus\left(\left.\left.\left(\mathcal{T}_{i}-\mathscr{U}\right)\right|_{a_{i}} \uplus \mathscr{U}\right|_{a}\right)\right|_{b \prec a} \uplus \cdots \uplus \mathcal{T}_{M}\right|_{a_{M}}, r\right\}\right|_{\substack{l_{i} \prec a_{i} \\(i=1, \ldots, M)}} .
$$

(ii) Any permutation $\boldsymbol{\sigma}^{\mathcal{F}}$ satisfying eq. (6.10) can be obtained by shuffling $\left.\boldsymbol{\zeta} \in \mathscr{U}\right|_{a}$ (a relative order of elements in $\mathscr{U}$ ) with $\gamma$ (a relative order of elements in $\mathscr{L}$ )

$$
\left.\gamma \in\left\{\left.\left.\left.\{2, \ldots, r-1\} ш \mathcal{T}_{1}\right|_{a_{1}} \uplus \cdots \uplus\left(\mathcal{T}_{i}-\mathscr{U}\right)\right|_{a_{i}} \uplus \cdots \uplus \mathcal{T}_{M}\right|_{a_{M}}\right\}\right|_{\substack{l_{i} \prec a_{i} \\(i=, \ldots, M)}}
$$

such that $\left(\sigma^{\mathcal{F}}\right)^{-1}(b)<\left(\sigma^{\mathcal{F}}\right)^{-1}(a)$. Thus all permutations $\boldsymbol{\sigma}^{\mathcal{F}}$ in eq. (6.6) satisfy $\boldsymbol{\sigma}^{\mathcal{F}} \in$ $\left.\{1, \boldsymbol{\gamma} \amalg \boldsymbol{\zeta}, r\}\right|_{\left(\sigma^{\mathcal{F}}\right)^{-1}(b)<\left(\sigma^{\mathcal{F}}\right)^{-1}(a)}$ for a given $\boldsymbol{\gamma}$ satisfying eq. (6.11) and a given $\left.\boldsymbol{\zeta} \in \mathscr{U}\right|_{a}$. 


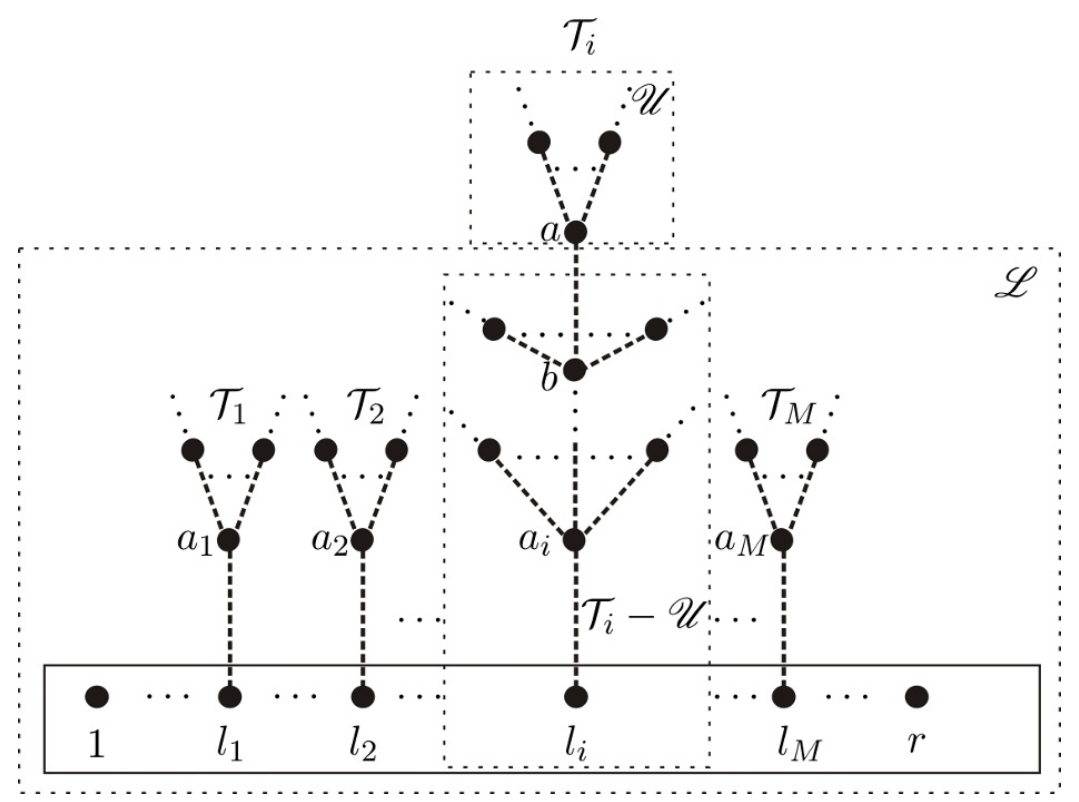

Figure 16. Permutations established by a graph with trees $\mathcal{T}_{1}, \mathcal{T}_{2}, \ldots, \mathcal{T}_{M}$ planted at roots $l_{1}, l_{2}, \ldots, l_{M} \in\{1, \ldots, r-1\}$ are given by eq. (6.10). The nearest-to-root nodes in these trees are correspondingly $a_{1}, a_{2}, \ldots, a_{M}$. The final upper block belongs to the tree $\mathcal{T}_{i}$ which is rooted at $l_{i} \in\{1, \ldots, r-1\}$. Nodes $b \in \mathscr{L}$ and $a \in \mathscr{U}$ are the two nodes that belong to distinct blocks and connected by a type-3 line. According to whether the nodes belong to the final upper or lower block, the tree $\mathcal{T}_{i}$ is divided into two parts $\mathscr{U}$ and $\mathcal{T}_{i}-\mathscr{U}$.

Having the above observations in hand, we reexpress eq. (6.6) by

$$
\begin{aligned}
& I[\mathscr{U}, \mathscr{L}] \\
& =\sum_{a \in \mathscr{U}} \sum_{\substack{b \in \mathscr{L} \\
b \neq r}}(-1)^{\mathcal{N}(\mathscr{L})+\mathcal{N}\left(\mathscr{U}_{a}\right)+\mathcal{S}\left(\mathscr{U}_{a}\right)+1}\left(k_{a} \cdot k_{b}\right) \sum_{\left.\boldsymbol{\zeta} \in \mathscr{U}\right|_{a}} \sum_{\gamma} \sum_{\boldsymbol{\sigma}} A\left(1,\left.\boldsymbol{\sigma} \in(\boldsymbol{\zeta} \boldsymbol{\gamma})\right|_{(\sigma)^{-1}(b)<(\sigma)^{-1}(a)}, r\right),
\end{aligned}
$$

where all $\boldsymbol{\gamma}$ satisfy eq. (6.11) and $\left.\boldsymbol{\zeta} \in \mathscr{U}\right|_{a}$ are the permutation for the final upper block when considering $a$ as the nearest to root node in $\mathscr{U}$. For a given $\mathscr{U}$ and a given node $a \in \mathscr{U}$, $\mathcal{N}(\mathscr{U})$ and $\mathcal{S}(\mathscr{U})$ are denoted by $\mathcal{N}\left(\mathscr{U}_{a}\right)$ and $\mathcal{S}\left(\mathscr{U}_{a}\right)$. Note that $\mathcal{N}(\mathscr{L})$ is independent of the choice of $b$ in the final lower block $\mathscr{L}$. Since the choices of nodes $a$ and $b$ are independent of each other, the above expression can be further arranged as

$$
\begin{aligned}
& I[\mathscr{U}, \mathscr{L}] \\
& =(-1)^{\mathcal{N}(\mathscr{L})} \sum_{\gamma}\left\{\sum_{a \in \mathscr{U}} \sum_{\left.\boldsymbol{\zeta} \in \mathscr{U}\right|_{a}}(-1)^{\mathcal{N}(\mathscr{U} a)+\mathcal{S}\left(\mathscr{U}_{a}\right)+1}\right. \\
& \left.\times\left[\sum_{\substack{b \in \mathscr{L} \\
b \neq r}}\left(k_{a} \cdot k_{b}\right) \sum_{\boldsymbol{\sigma}} A\left(1,\left.\boldsymbol{\sigma} \in(\boldsymbol{\zeta} \boldsymbol{\gamma})\right|_{(\sigma)^{-1}(b)<(\sigma)^{-1}(a)}, r\right)\right]\right\} .
\end{aligned}
$$


As we have shown in the example in section 4 , when we collect coefficients for any given permutation $\boldsymbol{\sigma} \in \boldsymbol{\gamma} \amalg \boldsymbol{\zeta}$ together, the expression in the square brackets in eq. (6.13) is given by

$$
\left(k_{a} \cdot Y_{a}(\boldsymbol{\sigma})\right) A(1, \boldsymbol{\sigma} \in(\boldsymbol{\zeta} \amalg \boldsymbol{\gamma}), r)
$$

Here $Y_{a}(\boldsymbol{\sigma})$ is the sum of all momenta of elements in $\gamma$ (the element 1 is also included) appearing on the 1.h.s. of the node $a$ in $\boldsymbol{\sigma}$. The factor $(-1)^{\mathcal{N}\left(\mathscr{U}_{a}\right)+\mathcal{S}\left(\mathscr{U}_{a}\right)+1}$ in eq. $(6.13)$ depends on the choice of $a$. Once the node $a \in \mathscr{U}$ (i.e. the node nearest to root in $\mathscr{U}$ ) under the first summation in the braces of eq. (6.13) is fixed, both the number $\mathcal{N}\left(\mathscr{U}_{a}\right)$ of arrows pointing away from the direction of root and the number $\mathcal{S}\left(\mathscr{U}_{a}\right)$ of spurious components in $\mathscr{U}$ are fixed. To analyze the relation between signs corresponding to distinct choices of $a$, we consider two adjacent nodes $c_{1}, c_{2} \in \mathscr{U}$ which can be connected via all three types of lines:

(i) If the line between $c_{1}$ and $c_{2}$ is a type-2 line, as shown by figure 17 (a), the factors for choosing $a=c_{1}$ and $a=c_{2}$ are related by flipping the sign, i.e. $(-1)^{\mathcal{N}\left(\mathscr{U} c_{1}\right)}=$ $(-1)^{\mathcal{N}\left(\mathscr{U}_{c_{2}}\right) \pm 1}$ because the difference of the numbers of all arrows pointing away from root for these two choices is 1 .

(ii) If $c_{1}$ and $c_{2}$ are two ends of a type-1 line or a type-3 line, as shown by figure 17 (b) and (c), the number of arrows that point away from root are same for $a=c_{1}$ and $a=c_{2}$. Thus $(-1)^{\mathcal{N}\left(\mathscr{C}_{c_{1}}\right)}=(-1)^{\mathcal{N}\left(\mathscr{U} c_{2}\right)}$. Nevertheless, we should also count the number $\mathcal{S}(\mathscr{U})$ of spurious chains in a (spurious) graph. Assume that the distance between $c_{1}$ and the (highest weight) node $h_{\rho(s)}$ is always larger than that between $c_{2}$ and $h_{\rho(s)}$. If $c_{1}, c_{2}$ are two end nodes of a type-1 (or type-3) line, the numbers of spurious components $\mathcal{S}(\mathscr{U})$ for these two cases always differ by 1 . Thus the factors $(-1)^{\mathcal{S}\left(\mathscr{U}_{a}\right)}$ for choosing $a=c_{1}$ and $a=c_{2}$ always have the opposite sign.

To sum up, the factors $(-1)^{\mathcal{N}(\mathscr{U} a)+\mathcal{S}\left(\mathscr{U}_{a}\right)+1}$ associated with graphs corresponding to $a=c_{1}$ and $a=c_{2}$, where $c_{1}, c_{2} \in \mathcal{U}$ are two adjacent nodes connected by an arbitrary type of line, always differ by a factor $(-1)$. Replacing the expression in the square brackets of eq. (6.13) by eq. (6.14) and considering the relative signs, we rewrite eq. (6.14) as

$$
I[\mathscr{U}, \mathscr{L}]=(-1)^{\mathcal{N}(\mathscr{L})+\mathcal{N}\left(\mathscr{U}_{c}\right)+\mathcal{S}\left(\mathscr{U}_{c}\right)+1} \sum_{\gamma}\left[\sum_{a \in \mathscr{U}} f^{a} \sum_{\left.\boldsymbol{\zeta} \in \mathscr{U}\right|_{a}} \sum_{\boldsymbol{\sigma}}\left(k_{a} \cdot Y_{a}(\boldsymbol{\sigma})\right) A(1, \boldsymbol{\sigma} \in(\boldsymbol{\zeta} \boldsymbol{\gamma}), r)\right],
$$

where the factor $(-1)^{\mathcal{N}(\mathscr{L})+\mathcal{N}\left(\mathscr{U}_{c}\right)+\mathcal{S}\left(\mathscr{U}_{c}\right)+1}$ for a fixed $c \in \mathscr{U}$ eq. (6.13) has been exacted out. The relative signs $f^{a}$ for choosing $a$ as other nodes can be fully fixed because the factor for any two adjacent choices of $a$ must be differ by a factor $(-1)$. In the next section, we will prove that for any given $\gamma$, the expression in the brackets of eq. (6.15) must vanish because it can always be written as a combination of BCJ relations. 


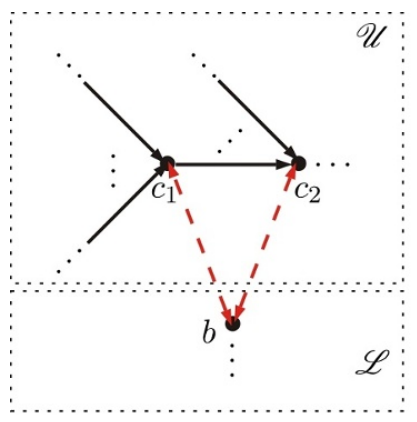

(a)

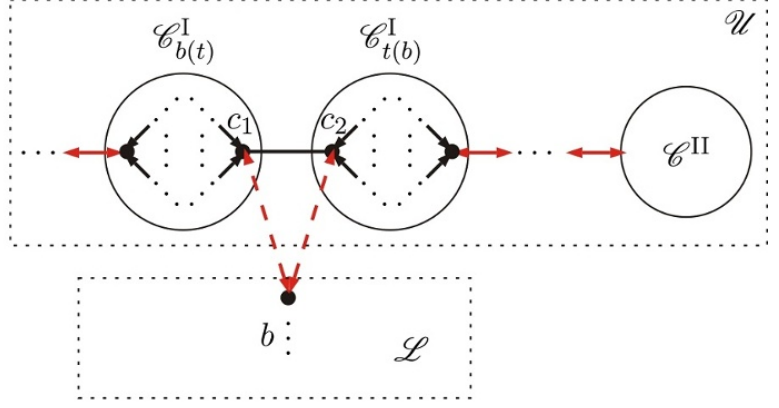

(b)

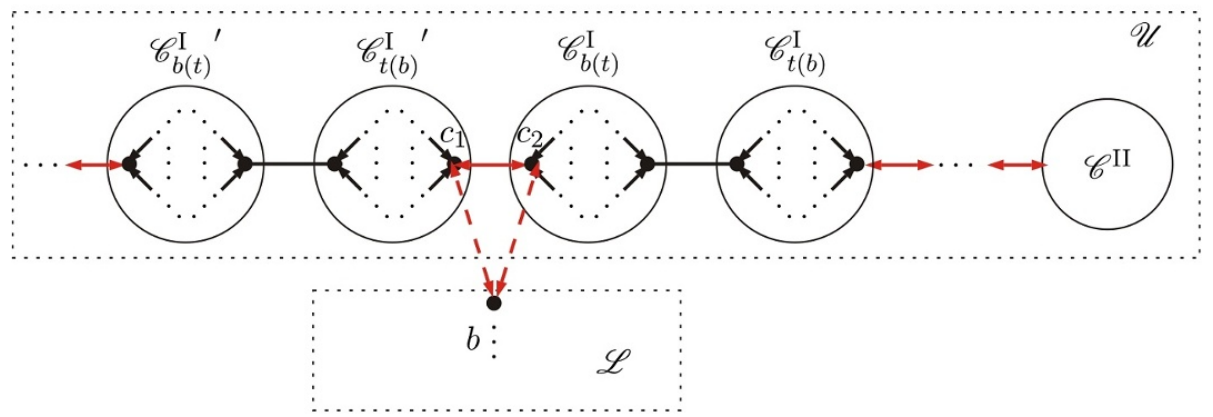

(c)

Figure 17. For any given configuration of the final upper and lower blocks $\mathscr{U}$ and $\mathscr{L}$, the factors

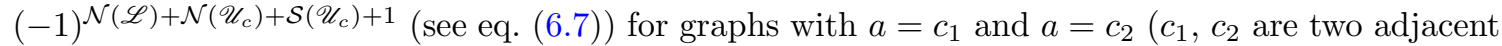
nodes in the final upper block) always have opposite signs. Particularly, the number $\mathcal{N}(\mathscr{L})$ is always independent of the choice of $a$. When we consider the number $\mathcal{N}\left(\mathscr{U}_{c}\right)+\mathcal{S}\left(\mathscr{U}_{c}\right)$, there are three possible cases: (i) In (a), $c_{1}$ and $c_{2}$ are two end nodes of a type- 3 line and they both belong to a same top or bottom side of some type-I component. The two graphs corresponding to $a=c_{1}$ and $a=c_{2}$ must have the same $\mathcal{S}\left(\mathscr{U}_{c}\right)$, but $\mathcal{N}\left(\mathscr{U}_{c_{1}}\right)=\mathcal{N}\left(\mathscr{U}_{c_{2}}\right)+1$. (ii) In (b), $c_{1}$ and $c_{2}$ are two end nodes of a type-1 line. Since a type-1 line does node carry arrow, we have $\mathcal{N}\left(\mathscr{U}_{c_{1}}\right)=\mathcal{N}\left(\mathscr{U}_{c_{2}}\right)$. The component $\mathscr{C}^{\mathrm{I}}$ is a spurious one for the graph with $a=c_{2}$ but not spurious for the graph with $a=c_{1}$, thus $\mathcal{S}\left(\mathscr{U}_{c_{1}}\right)=\mathcal{S}\left(\mathscr{U}_{c_{2}}\right)-1$. (iii) Similarly, in (c), where $c_{1}$ and $c_{2}$ are two end nodes of a type-3 line, we have $\mathcal{N}\left(\mathscr{U}_{c_{1}}\right)=\mathcal{N}\left(\mathscr{U}_{c_{2}}\right)$ and $\mathcal{S}\left(\mathscr{U}_{c_{1}}\right)=\mathcal{S}\left(\mathscr{U}_{c_{2}}\right)+1$.

\section{Graph-based BCJ relation as a combination of traditional BCJ relations}

In this section, we introduce the following graph-based BCJ relation ${ }^{8}$

$$
\sum_{a \in \mathcal{T}} f^{a} \sum_{\left.\boldsymbol{\zeta} \in \mathcal{T}\right|_{a}} \sum_{\sigma}\left[k_{a} \cdot Y_{a}(\boldsymbol{\sigma})\right] A(1, \boldsymbol{\sigma} \in(\boldsymbol{\zeta} \amalg \boldsymbol{\gamma}), r)=0
$$

Here, $\gamma$ is an arbitrary permutation of elements in $\{2, \ldots, r-1\}$ and $\mathcal{T}$ is an arbitrary connected tree graph. We use $\left.\mathcal{T}\right|_{a}$ to denote the relative orders between nodes of $\mathcal{T}$ when the node $a$ is the leftmost one (see section 6.3). For a given graph $\mathcal{T}$, the factor $f^{a}$ is a

\footnotetext{
${ }^{8}$ Similar relations have also been discussed by Chen, Johanssion, Teng and Wang [32] via kinematic algebra.
} 
relative sign depending on the choice of $a$. This factor is fixed as follows: (i) Choose an arbitrary node $c$ and require $f^{c}=1$, (ii) For arbitrary two adjacent nodes $c_{1}$ and $c_{2}$, we have $f^{c_{1}}=-f^{c_{2}}$. In the following, we use $\mathcal{B}_{G}^{c}(1|\mathcal{T}, \gamma| r)$ to stand for the l.h.s. of eq. (7.1) with choosing $f^{c}=1(c \in \mathcal{T})$. We will prove that the $\mathcal{B}_{G}^{c}(1|\mathcal{T}, \gamma| r)$ (thus eq. (6.15) and eq. (6.5)) is a combination of the l.h.s. of traditional BCJ relations eq. (2.10) (see eq. (2.11)). As a result, the gauge invariance induced identity eq. (2.9) can always be expanded in terms of traditional BCJ relations.

\subsection{Examples}

Now we present several examples for the graph-based BCJ relation eq. (7.1).

Example-1. The simplest example is that the tree graph $\mathcal{T}$ consists of only a single node $h_{1}$. The l.h.s. of eq. (7.1) is nothing but the 1.h.s. of a fundamental BCJ relation

$$
\mathcal{B}_{G}^{h_{1}}\left(1\left|\bullet^{h_{1}}, \boldsymbol{\gamma}\right| r\right)=\mathcal{B}\left(1\left|\left\{h_{1}\right\}, \gamma\right| r\right)
$$

Example-2. The next simplest example is that $\mathcal{T}$ consists of two nodes $h_{1}$ and $h_{2}$ with one (dashed) line between them. The 1.h.s. of eq. (7.1) in this case reads

$$
\begin{aligned}
& \mathcal{B}_{G}^{h_{1}}\left(1\left|\stackrel{h_{1} \ldots \ldots h_{2}}{h_{2}}, \gamma\right| r\right)=\sum_{\uplus}\left(k_{h_{1}} \cdot Y_{h_{1}}(\uplus)\right) A\left(1,\left\{h_{1}, h_{2}\right\} \amalg \boldsymbol{\gamma}, r\right) \\
& -\sum_{\varpi}\left(k_{h_{2}} \cdot Y_{h_{2}}(\uplus)\right) A\left(1,\left\{h_{2}, h_{1}\right\} \amalg \boldsymbol{\gamma}, r\right) .
\end{aligned}
$$

Notice that the last term can be replaced by

$$
\mathcal{B}_{G}^{h_{2}}\left(\left.1\right|_{\bullet} ^{h_{2}},\left\{h_{1}\right\} ш \gamma \mid r\right)-\sum_{\varpi}\left(k_{h_{2}} \cdot X_{h_{2}}(ш)\right) A\left(1,\left\{h_{1}, h_{2}\right\} \amalg \gamma, r\right)
$$

The second term of the above equation together with the first term of eq. (7.3) produces the l.h.s. of the traditional BCJ relation eq. (2.10) with $\beta=\left\{h_{1}, h_{2}\right\}$, i.e. $\mathcal{B}\left(1\left|\left\{h_{1}, h_{2}\right\}, \gamma\right| r\right)$. Thus eq. (7.3) is finally given by

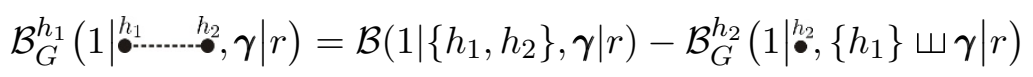

$$
\begin{aligned}
& =\mathcal{B}\left(1\left|\left\{h_{1}, h_{2}\right\}, \gamma\right| r\right)-\mathcal{B}\left(1\left|\left\{h_{2}\right\},\left\{h_{1}\right\} ш \gamma\right| r\right),
\end{aligned}
$$

which is a combination of the l.h.s. of traditional BCJ relations. It is worth pointing out that the above expression is not unique. When exchanging the roles of $h_{1}$ and $h_{2}$ in eq. (7.5), we get another equivalent expansion

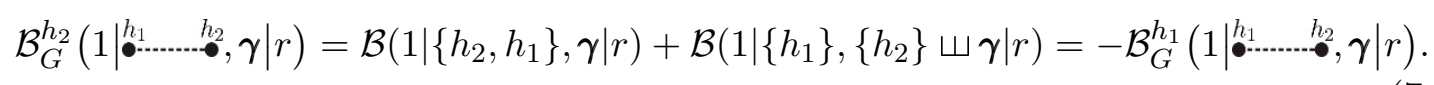




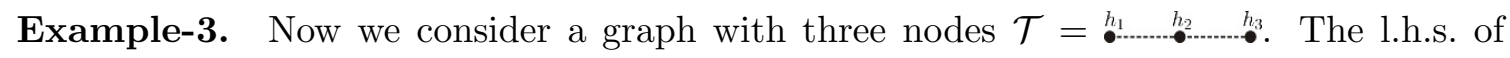
eq. (7.1) for this graph reads

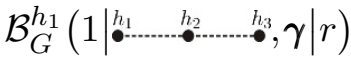

$$
\begin{aligned}
& =\sum_{\varpi}\left(k_{h_{1}} \cdot Y_{h_{1}}(\uplus)\right) A\left(1,\left\{h_{1}, h_{2}, h_{3}\right\} \amalg \boldsymbol{\gamma}, r\right) \\
& -\left[\sum_{\uplus_{1}, \uplus_{2}}\left(k_{h_{2}} \cdot Y_{h_{2}}\left(\varpi_{1}, \varpi_{2}\right)\right) A\left(1,\left\{h_{2},\left\{h_{1}\right\} \varpi_{1}\left\{h_{3}\right\}\right\} \varpi_{2} \gamma, r\right)\right. \\
& \left.-\sum_{\uplus}\left(k_{h_{3}} \cdot Y_{h_{3}}(\uplus)\right) A\left(1,\left\{h_{3}, h_{2}, h_{1}\right\} ш \boldsymbol{\gamma}, r\right)\right] .
\end{aligned}
$$

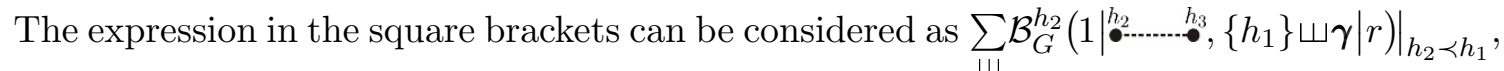
where $h_{2} \prec h_{1}$ for a given permutation $\boldsymbol{\xi}$ means that $\xi^{-1}\left(h_{2}\right)<\xi^{-1}\left(h_{1}\right)$. When the first equality of eq. (7.5) in example-2 is applied, $\left.\sum_{\Psi} \mathcal{B}_{G}^{h_{2}}\left(1||_{\bullet-\ldots . .}^{h_{2}},\left\{h_{1}\right\} \amalg \gamma \mid r\right)\right|_{h_{2} \prec h_{1}}$ turns to

$$
\begin{aligned}
& \left.\sum_{\uplus} \mathcal{B}_{G}^{h_{2}}\left(1||_{\bullet \cdots \cdots}^{h_{2}} \quad h_{3},\left\{h_{1}\right\} \amalg \gamma \mid r\right)\right|_{h_{2} \prec h_{1}} \\
& =\left.\sum_{\uplus} \mathcal{B}\left(1\left|\left\{h_{2}, h_{3}\right\},\left\{h_{1}\right\} \amalg \boldsymbol{\gamma}\right| r\right)\right|_{h_{2} \prec h_{1}}-\sum_{\uplus} \mathcal{B}_{G}^{h_{3}}\left(1||^{h_{3}},\left\{h_{2}, h_{1}\right\} \amalg \boldsymbol{\gamma} \mid r\right) \\
& =\sum_{\varpi} \mathcal{B}\left(1\left|\left\{h_{2}, h_{3}\right\},\left\{h_{1}\right\} ш \boldsymbol{\gamma}\right| r\right)-\left.\mathcal{B}\left(1\left|\left\{h_{2}, h_{3}\right\},\left\{h_{1}\right\} \amalg \boldsymbol{\gamma}\right| r\right)\right|_{h_{1} \prec h_{2}}-\sum_{\varpi} \mathcal{B}_{G}^{h_{3}}\left(\left.1\right|^{h_{3}},\left\{h_{2}, h_{1}\right\} \amalg \boldsymbol{\gamma} \mid r\right) .
\end{aligned}
$$

The first term and the third term on the last line are just a combination of 1.h.s. of BCJ relations with $\beta=\left\{h_{2}, h_{3}\right\}$ and $\beta=\left\{h_{3}\right\}$ respectively. The sum of the second term on the last line of the above equation and the first term in eq. (7.7) is nothing but the l.h.s. of traditional BCJ relation eq. (2.10) with $\beta=\left\{h_{1}, h_{2}, h_{3}\right\}$. Hence eq. (7.7) is finally expanded into the following two parts

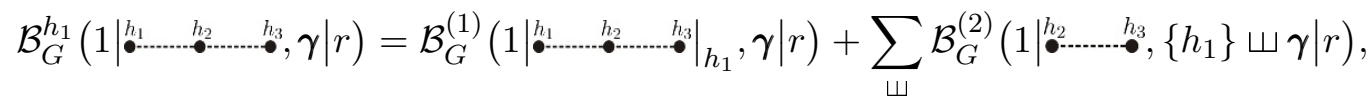

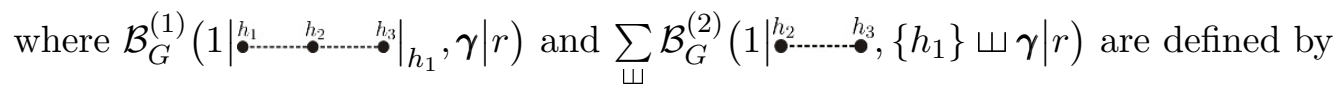

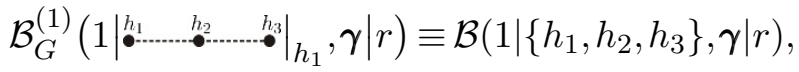

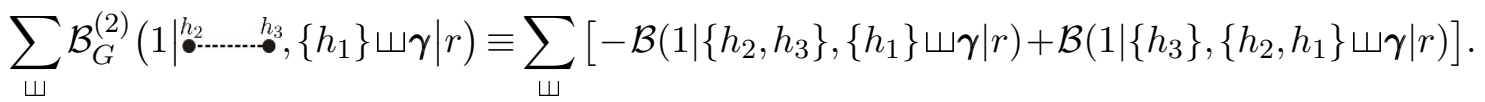

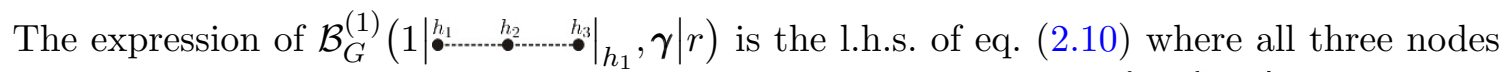

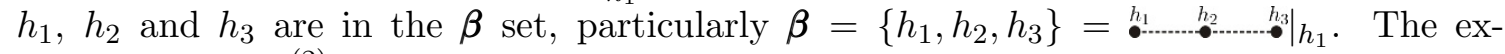
pression of $\sum_{\Psi} \mathcal{B}_{G}^{(2)}\left(\left.1\right|_{\bullet} ^{h_{2}} h_{3},\left\{h_{1}\right\} \Psi \gamma \mid r\right)$ is a combination of BCJ relations with fewer $\beta$ 's. The node $h_{1}$ is always considered as an element of the $\boldsymbol{\alpha}$ set in the expression of $\sum_{\uplus} \mathcal{B}_{G}^{(2)}\left(1||^{h_{2}}-h_{3} \cdot \bullet,\left\{h_{1}\right\} \amalg \gamma \mid r\right)$. 


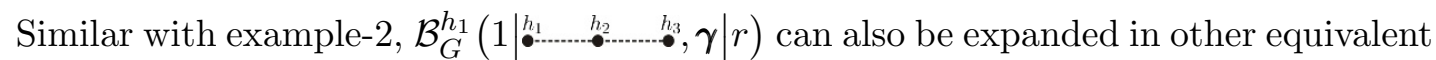
forms. For example, we consider $\mathcal{B}_{G}^{h_{2}}\left(1\left|\begin{array}{ccc}h_{1} & h_{2} & h_{3} \\ \bullet & h_{3} & \boldsymbol{h}\end{array}\right| r\right)$ which is expressed by eq. (7.7) associated with a minus sign. We keep the first term (the term where $h_{2}$ is considered as the leftmost node in the graph $\mathcal{T}$ ) in the square brackets of eq. (7.7) and rewrite other two terms according to eq. (7.2) as follows

$$
\begin{aligned}
& \sum_{\varpi}\left(k_{h_{1}} \cdot Y_{h_{1}}(ш)\right) A\left(1,\left\{h_{1}, h_{2}, h_{3}\right\} ш \gamma, r\right) \\
& =\sum_{\varpi} \mathcal{B}_{G}^{h_{1}}\left(\left.1\right|^{h_{1}},\left\{h_{2}, h_{3}\right\} \amalg \gamma \mid r\right)-\sum_{\uplus_{1}, \uplus_{2}}\left(k_{h_{1}} \cdot X_{h_{1}}\right) A\left(1,\left\{h_{2},\left\{h_{1}\right\} \varpi_{1}\left\{h_{3}\right\}\right\} \varpi_{2} \gamma, r\right), \\
& \sum_{ш}\left(k_{h_{3}} \cdot Y_{h_{3}}(ш)\right) A\left(1,\left\{h_{3}, h_{2}, h_{1}\right\} ш \gamma, r\right) \\
& =\sum_{\varpi} \mathcal{B}_{G}^{h_{3}}\left(\left.1\right|_{\bullet} ^{h_{3}},\left\{h_{2}, h_{1}\right\} ш \gamma \mid r\right)-\sum_{\varpi_{1}, \uplus_{2}}\left(k_{h_{3}} \cdot X_{h_{3}}\right) A\left(1,\left\{h_{2},\left\{h_{1}\right\} \varpi_{1}\left\{h_{3}\right\}\right\} \varpi_{2} \gamma, r\right) .
\end{aligned}
$$

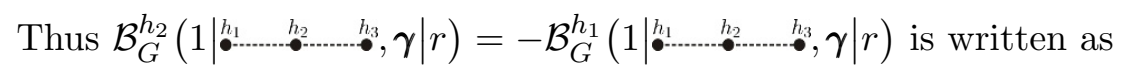

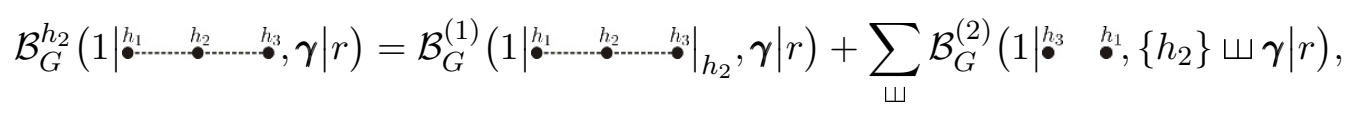

where

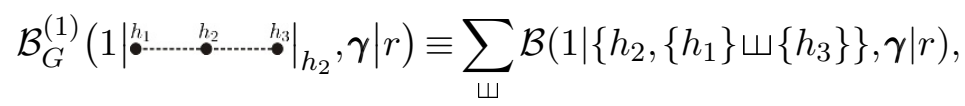

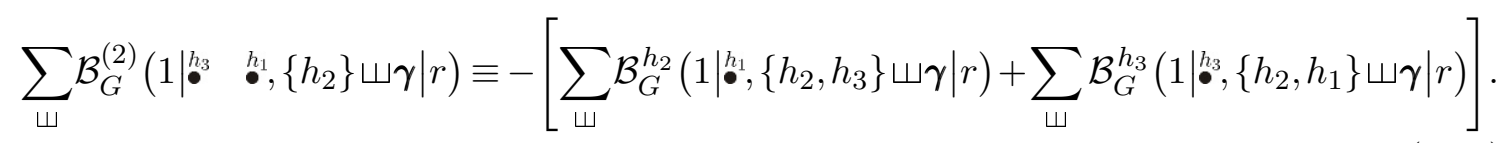

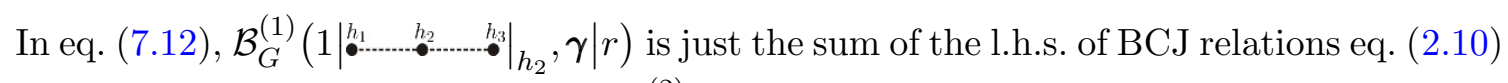
with $\beta \in\left\{h_{2}, h_{1} \amalg h_{3}\right\}$. The term $\sum_{\Psi} \mathcal{B}_{G}^{(2)}\left(\left.1\right|^{h_{3}} \quad h_{1},\left\{h_{2}\right\} ш \gamma \mid r\right)$ in eq. (7.12) is a combination of the 1.h.s. of graph-based BCJ relations with one-node subtrees (here, $h_{2}$ is in the $\boldsymbol{\alpha}$ set), thus it is also a combination of traditional BCJ relations (according to eq. (7.2)).

Example-4. The first example of graphs with complex chains is the star graph with four nodes. The 1.h.s. of the graph-based-BCJ relation eq. (7.1) for this example reads:

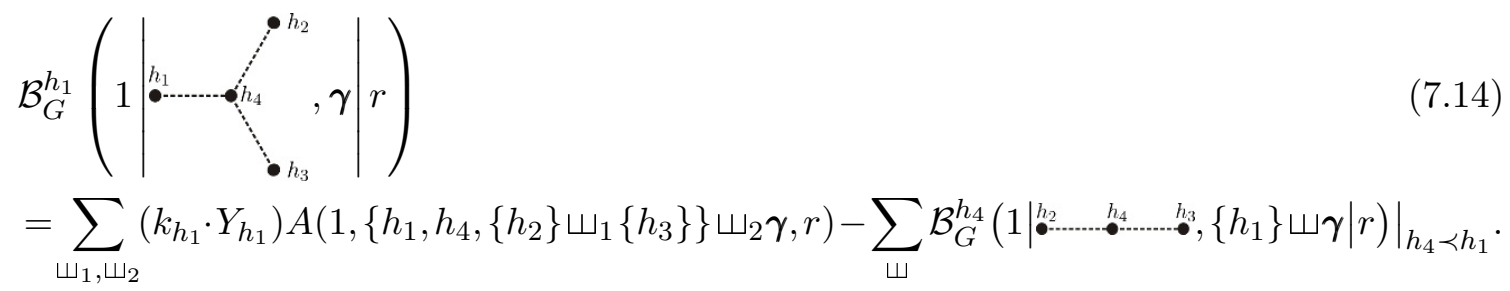

According to eq. (7.12), the last term is given by

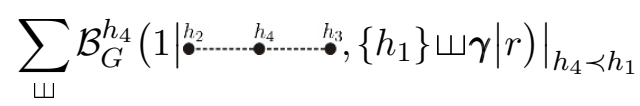

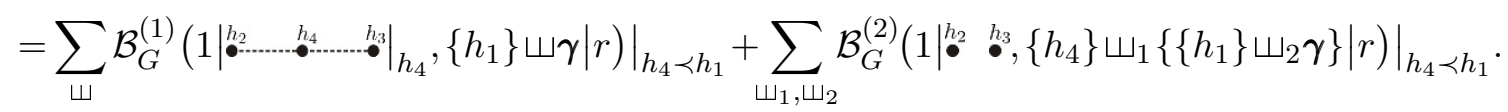


From eq. (7.13), we know that the second term of the above equation is expressed in terms of the l.h.s. of those BCJ relations where $h_{4}$ belongs to the $\boldsymbol{\alpha}$ set. Since $h_{1}$ is also in the $\boldsymbol{\alpha}$ set (of the traditonal BCJ expansion) of the second term, $\sum_{\uplus_{1}, \uplus_{2}} \mathcal{B}_{G}^{(2)}\left(\left.1\right|_{\bullet} ^{h_{2}} \stackrel{\bullet}{\bullet}^{h_{3}},\left\{h_{4}\right\} \varpi_{1}\right.$ $\left.\left\{\left\{h_{1}\right\} \varpi_{2} \gamma\right\} \mid r\right)\left.\right|_{h_{4} \prec h_{1}}$ is already a combination of the 1.h.s. of BCJ relations where both $h_{4}$ and $h_{1}$ belong to $\boldsymbol{\alpha}$ set and satisfy $h_{4} \prec h_{1}$. The first term in eq. (7.15) reads

$$
\sum_{\uplus_{1}, \varpi_{2}} \mathcal{B}\left(1\left|\left\{h_{4},\left\{h_{2}\right\} \varpi_{1}\left\{h_{3}\right\}\right\},\left\{h_{1}\right\} \varpi_{2} \gamma\right| r\right)-\left.\sum_{\uplus_{1}, \varpi_{2}} \mathcal{B}\left(1\left|\left\{h_{4},\left\{h_{2}\right\} \varpi_{1}\left\{h_{3}\right\}\right\},\left\{h_{1}\right\} \varpi_{2} \gamma\right| r\right)\right|_{h_{1} \prec h_{4}} .
$$

The sum of the last term in eq. (7.16) and the first term in eq. (7.14) is

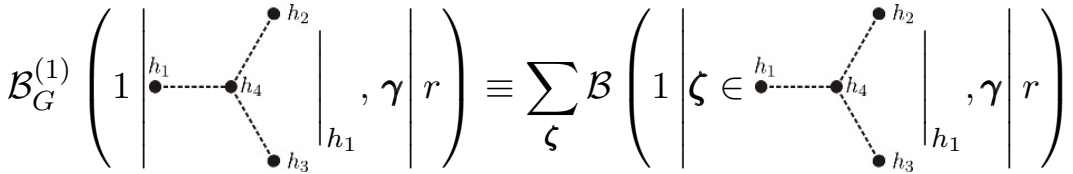

$$
\begin{aligned}
& =\sum_{\varpi} \mathcal{B}\left(1\left|\left\{h_{1}, h_{4},\left\{h_{2}\right\} \amalg\left\{h_{3}\right\}\right\}, \gamma\right| r\right),
\end{aligned}
$$

which is a combination of the l.h.s. of traditional BCJ relations with all the four nodes belonging to the $\beta$ set. The sum of the first term in eq. (7.16) and the last term in eq. (7.15) defines

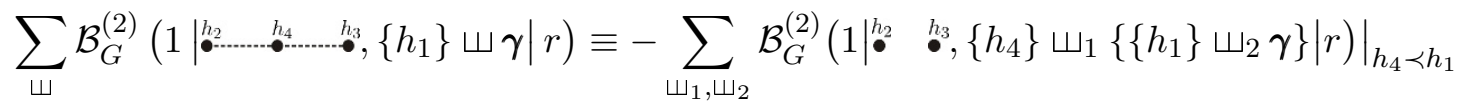

$$
\begin{aligned}
& -\sum_{\amalg} \mathcal{B}\left(1\left|\left\{h_{4},\left\{h_{2}\right\} \uplus\left\{h_{3}\right\}\right\},\left\{h_{1}\right\} ш \gamma\right| r\right),
\end{aligned}
$$

which is a combination of the l.h.s. of traditional BCJ relations where $h_{1}$ is an element of $\alpha$ set.

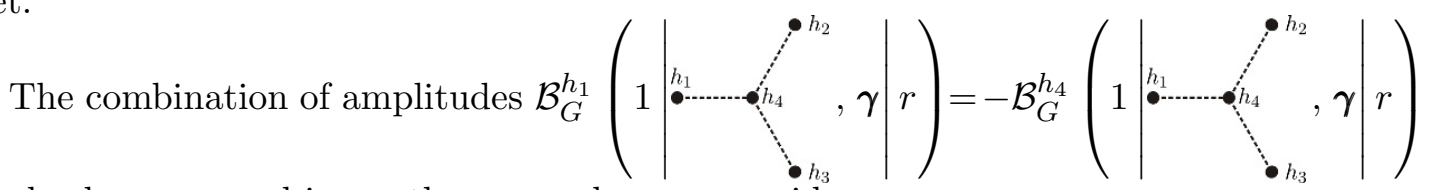
can also be expressed in another way when we consider

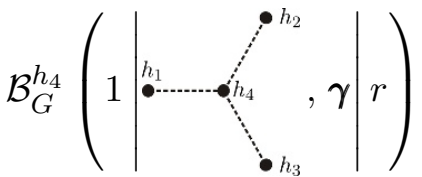

$$
\begin{aligned}
& =\sum_{\uplus_{1}, \uplus_{2}, \uplus_{3}}\left(k_{h_{4}} \cdot Y_{h_{4}}\right) A\left(1,\left\{h_{4},\left\{h_{1}\right\} \uplus_{1}\left\{h_{2}\right\} \varpi_{2}\left\{h_{3}\right\}\right\} \uplus_{3} \gamma, r\right) \\
& -\left[\left.\sum_{\varpi_{1}, \varpi_{2}} \mathcal{B}_{G}^{h_{4}}\left(\left.1\right|^{h_{1}},\left\{h_{4},\left\{h_{2}\right\} \uplus_{1}\left\{h_{3}\right\}\right\} \uplus_{2} \gamma \mid r\right)\right|_{h_{1} \prec h_{4}}\right. \\
& +\left.\sum_{\uplus_{1}, \uplus_{2}} \mathcal{B}_{G}^{h_{4}}\left(1||_{\bullet}^{h_{2}},\left\{h_{4},\left\{h_{1}\right\} \uplus_{1}\left\{h_{3}\right\}\right\} \varpi_{2} \gamma \mid r\right)\right|_{h_{2} \prec h_{4}} \\
& \left.+\left.\sum_{\uplus_{1}, \uplus_{2}} \mathcal{B}_{G}^{h_{4}}\left(\left.1\right|_{\bullet} ^{h_{3}},\left\{h_{4},\left\{h_{1}\right\} \uplus_{1}\left\{h_{2}\right\}\right\} \uplus_{2} \gamma \mid r\right)\right|_{h_{3} \prec h_{4}}\right] .
\end{aligned}
$$


Applying eq. (7.2), we rewrite the last three terms of the above equation as follows

$$
\begin{aligned}
& \left.\sum_{\uplus_{1}, \uplus_{2}} \mathcal{B}_{G}^{h_{4}}\left(1||^{h_{1}},\left\{h_{4},\left\{h_{2}\right\} \varpi_{1}\left\{h_{3}\right\}\right\} \varpi_{2} \gamma \mid r\right)\right|_{h_{1} \prec h_{4}} \\
& =\sum_{\varpi_{1}, \uplus_{2}} \mathcal{B}\left(1\left|\left\{h_{1}\right\},\left\{h_{4},\left\{h_{2}\right\} \varpi_{1}\left\{h_{3}\right\}\right\} \varpi_{2} \gamma\right| r\right)-\left.\sum_{\uplus_{1}, \uplus_{2}} \mathcal{B}\left(1\left|\left\{h_{1}\right\},\left\{h_{4},\left\{h_{2}\right\} \varpi_{1}\left\{h_{3}\right\}\right\} \varpi_{2} \gamma\right| r\right)\right|_{h_{1} \succ h_{4}}, \\
& \left.\sum_{\uplus_{1}, \uplus_{2}} \mathcal{B}_{G}^{h_{4}}\left(1\left|h^{h_{2}},\left\{h_{4},\left\{h_{1}\right\} \varpi_{1}\left\{h_{3}\right\}\right\} \varpi_{2} \gamma\right| r\right)\right|_{h_{2} \prec h_{4}} \\
& =\sum_{\varpi_{1}, \varpi_{2}} \mathcal{B}\left(1\left|\left\{h_{2}\right\},\left\{h_{4},\left\{h_{1}\right\} \varpi_{1}\left\{h_{3}\right\}\right\} \varpi_{2} \gamma\right| r\right)-\left.\sum_{\uplus_{1}, \varpi_{2}} \mathcal{B}\left(1\left|\left\{h_{2}\right\},\left\{h_{4},\left\{h_{1}\right\} \varpi_{1}\left\{h_{3}\right\}\right\} \varpi_{2} \gamma\right| r\right)\right|_{h_{2} \succ h_{4}}, \\
& \left.\sum_{\varpi_{1}, \uplus_{2}} \mathcal{B}_{G}^{h_{4}}\left(1||_{\bullet}^{h_{3}},\left\{h_{4},\left\{h_{1}\right\} \varpi_{1}\left\{h_{2}\right\}\right\} \varpi_{2} \gamma \mid r\right)\right|_{h_{3} \prec h_{4}} \\
& =\sum_{\varpi_{1}, \uplus_{2}} \mathcal{B}\left(1\left|\left\{h_{3}\right\},\left\{h_{4},\left\{h_{1}\right\} \varpi_{1}\left\{h_{2}\right\}\right\} \varpi_{2} \gamma\right| r\right)-\left.\sum_{\uplus_{1}, \uplus_{2}} \mathcal{B}\left(1\left|\left\{h_{3}\right\},\left\{h_{4},\left\{h_{1}\right\} \varpi_{1}\left\{h_{2}\right\}\right\} \varpi_{2} \gamma\right| r\right)\right|_{h_{3} \succ h_{4}} .
\end{aligned}
$$

The sum of the last terms of the above equations and the first term of eq. (7.19) gives rise

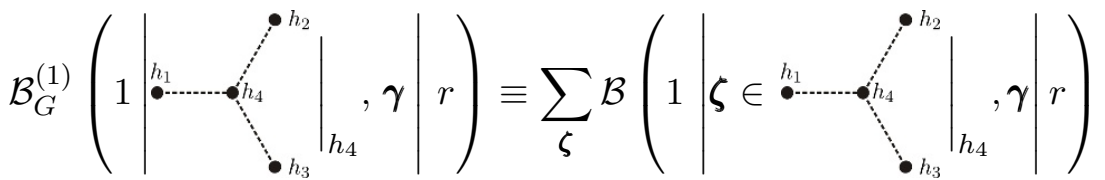

$$
\begin{aligned}
& =\sum_{\uplus_{1}, \uplus_{2}} \mathcal{B}\left(1\left|\left\{h_{4},\left\{h_{1}\right\} \varpi_{1}\left\{h_{2}\right\} \varpi_{2}\left\{h_{3}\right\}\right\}, \gamma\right| r\right),
\end{aligned}
$$

which is a combination of the l.h.s. of BCJ relations eq. (2.10) with all nodes are in the $\beta$ set. The sum of other contributions are collected as

$$
\begin{aligned}
& \sum_{ш} \mathcal{B}_{G}^{(2)}\left(1||_{\bullet}^{h_{1}} \quad \stackrel{h}{2}^{\circ} \quad \stackrel{h_{3}}{\bullet},\left\{h_{4}\right\} \amalg \boldsymbol{\gamma} \mid r\right) \\
& \equiv \sum_{\uplus_{1}, \uplus_{2}} \mathcal{B}\left(1\left|\left\{h_{1}\right\},\left\{h_{4},\left\{h_{2}\right\} \varpi_{1}\left\{h_{3}\right\}\right\} \varpi_{2} \gamma\right| r\right)+\sum_{\varpi_{1}, \varpi_{2}} \mathcal{B}\left(1\left|\left\{h_{2}\right\},\left\{h_{4},\left\{h_{1}\right\} \varpi_{1}\left\{h_{3}\right\}\right\} \varpi_{2} \gamma\right| r\right) \\
& +\sum_{\uplus_{1}, \uplus_{2}} \mathcal{B}\left(1\left|\left\{h_{3}\right\},\left\{h_{4},\left\{h_{1}\right\} \varpi_{1}\left\{h_{2}\right\}\right\} \varpi_{2} \gamma\right| r\right),
\end{aligned}
$$

which is a combination of the l.h.s. of BCJ relations eq. (2.10) where $h_{4}$ always belongs to the $\boldsymbol{\alpha}$ set.

\subsection{The general proof}

In general, the l.h.s. of graph-based BCJ relation eq. (7.1) for an arbitrary tree $\mathcal{T}$ can be expanded in terms of the l.h.s. of BCJ relations eq. (2.10). Particularly, we pick out an arbitrary node $a \in \mathcal{T}$ and assume that there are $N$ subtrees attached to $a$, say $\mathcal{T}_{a}^{1}$, $\mathcal{T}_{a}^{2}, \ldots, \mathcal{T}_{a}^{N}$. The corresponding nodes adjacent to $a$ in $\mathcal{T}_{a}^{1}, \mathcal{T}_{a}^{2}, \ldots, \mathcal{T}_{a}^{N}$ are $a_{1}, a_{2}, \ldots, a_{N}$. We will prove that the l.h.s. of eq. (7.1) can be expanded as

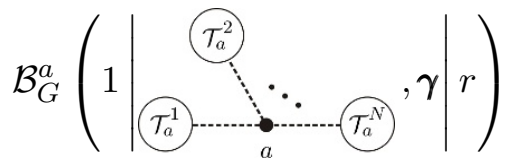

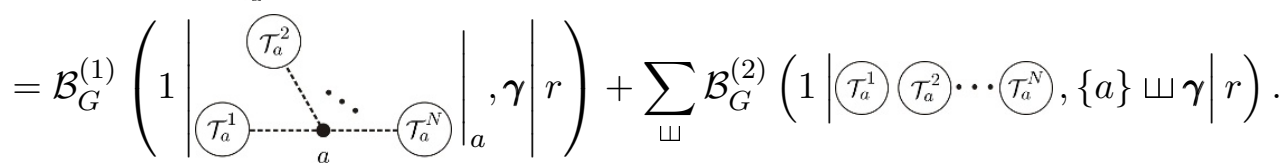




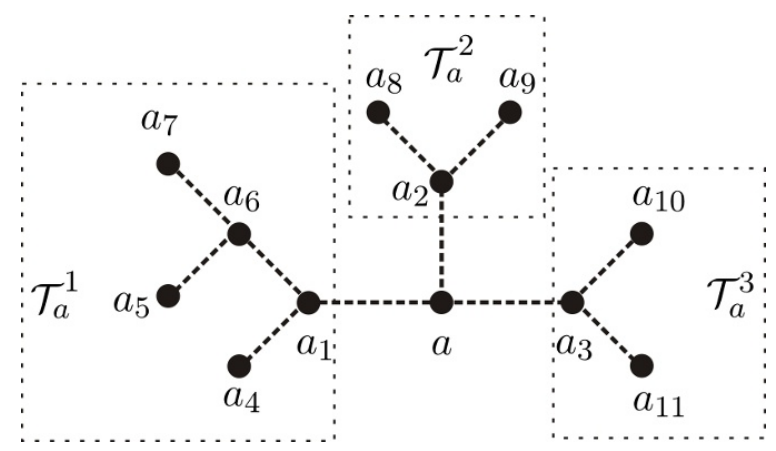

Figure 18. A tree graph where $a$ is connected with three subtrees $\mathcal{T}_{a}^{1}, \mathcal{T}_{a}^{2}$ and $\mathcal{T}_{a}^{3}$.

The first term of eq. (7.26) is the following combination

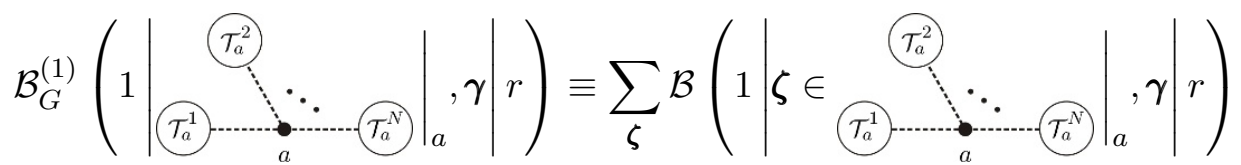

in which all nodes of $\mathcal{T}$ are in the $\beta$ set. The second term of eq. (7.26) is a proper combination of BCJ relations eq. (7.1) where the node $a$ is always in the $\boldsymbol{\alpha}$ set. The explicit expression of this part can be determined recursively. In the following, we will assume eq. (7.26) holds for all subtrees $\mathcal{T}_{a}^{1}, \mathcal{T}_{a}^{2}, \ldots, \mathcal{T}_{a}^{N}$ and prove eq. (7.26) by induction.

To prove eq. (7.26), we first show that $\mathcal{B}_{G}^{a}(1|\mathcal{T}, \gamma| r)$ can be expressed by those $\mathcal{B}_{G}^{a}$ 's with subtrees $\mathcal{T}_{a}^{1}, \ldots, \mathcal{T}_{a}^{N}$ connecting to the node $a$. Let us explain this point explicitly by considering the example with $\mathcal{T}=$ figure 18 . According to the definition, $\mathcal{B}_{G}^{a}(1 \mid \mathcal{T}=$ figure $18, \gamma \mid r)$ can be written as

$\mathcal{B}_{G}^{a}(1 \mid \mathcal{T}=$ Fig. $18, \boldsymbol{\gamma} \mid r)=\left[\sum_{\boldsymbol{\sigma}} \sum_{\left.\boldsymbol{\zeta} \in \mathcal{T}\right|_{a}}\left(k_{a} \cdot Y_{a}\right)+\sum_{i=1}^{3} \sum_{c \in \mathcal{T}_{a}^{i}} f^{c} \sum_{\boldsymbol{\sigma}} \sum_{\left.\boldsymbol{\zeta} \in \mathcal{T}\right|_{c}}\left(k_{c} \cdot Y_{c}\right)\right] A(1, \boldsymbol{\sigma} \in(\boldsymbol{\zeta} \boldsymbol{\gamma}), r)$.

Here $a_{1}, a_{2}$ and $a_{3}$ are the nearest-to- $a$ nodes which belong to substructures $\mathcal{T}_{a}^{1}, \mathcal{T}_{a}^{2}$ and $\mathcal{T}_{a}^{3}$ correspondingly. The total contributions from nodes in $\mathcal{T}_{a}^{1}$ (i.e. contributions with $i=1$ in the second term of eq. (7.28)) is given by

$$
\sum_{i \in \mathcal{T}_{a}^{1}} f^{i} \sum_{\left.\boldsymbol{\zeta}^{(1)} \in \mathcal{T}_{a}^{1}\right|_{i}}\left[\sum_{\left.\boldsymbol{\zeta}^{(2)} \in \mathcal{T}_{a}^{2}\right|_{a_{2}}} \sum_{\left.\boldsymbol{\zeta}^{(3)} \in \mathcal{T}_{a}^{3}\right|_{a_{3}}} \sum_{ш} \sum_{\boldsymbol{\sigma}}\left(k_{i} \cdot Y_{i}\right) A\left(1,\left.\boldsymbol{\sigma} \in\left(\boldsymbol{\zeta}^{(1)} \uplus^{\prime}\left\{a, \boldsymbol{\zeta}^{(2)} \uplus \boldsymbol{\zeta}^{(3)} ш \boldsymbol{\gamma}\right\}\right)\right|_{a_{1} \prec a}, r\right)\right],
$$

where $\left.\mathcal{T}_{a}^{2}\right|_{a_{2}}=\left\{a_{2},\left\{a_{8}\right\} \uplus\left\{a_{9}\right\}\right\},\left.\mathcal{T}_{a}^{3}\right|_{a_{3}}=\left\{a_{3},\left\{a_{10}\right\} \uplus\left\{a_{11}\right\}\right\}$. Permutations $\left.\mathcal{T}_{a}^{1}\right|_{i}$ in eq. (7.29) are those established by the subgraph $\mathcal{T}_{a}^{1}$ when $i \in \mathcal{T}_{a}^{1}$ is considered as the leftmost element. Specifically,

$$
\begin{aligned}
& \left.\mathcal{T}_{a}^{1}\right|_{a_{1}}=\left\{a_{1},\left\{a_{4}\right\} \uplus\left\{a_{6},\left\{a_{5}\right\} \amalg\left\{a_{7}\right\}\right\}\right\},\left.\quad \mathcal{T}_{a}^{1}\right|_{a_{4}}=\left\{a_{4}, a_{1}, a_{6},\left\{a_{5}\right\} \sqcup\left\{a_{7}\right\}\right\} \\
& \left.\mathcal{T}_{a}^{1}\right|_{a_{5}}=\left\{a_{5}, a_{6},\left\{a_{7}\right\} \uplus\left\{a_{1}, a_{4}\right\}\right\},\left.\quad \mathcal{T}_{a}^{1}\right|_{a_{6}}=\left\{a_{6},\left\{a_{5}\right\} \amalg\left\{a_{7}\right\} \amalg\left\{a_{1}, a_{4}\right\}\right\}, \\
& \left.\mathcal{T}_{a}^{1}\right|_{a_{7}}=\left\{a_{7}, a_{6},\left\{a_{5}\right\} \amalg\left\{a_{1}, a_{4}\right\}\right\} \text {. }
\end{aligned}
$$


For fixed permutations $\boldsymbol{\zeta}^{(2)}, \boldsymbol{\zeta}^{(3)}$ and a given permutation $\boldsymbol{\xi} \in\left\{a, \boldsymbol{\zeta}^{(2)} ш \boldsymbol{\zeta}^{(3)}\right\} ш \boldsymbol{\gamma}$, terms in eq. (7.29) are collected as

$$
\sum_{i \in \mathcal{T}_{a}^{1}} f^{i} \sum_{\left.\boldsymbol{\zeta}^{(1)} \in \mathcal{T}_{a}^{1}\right|_{i}} \sum_{\boldsymbol{\sigma}}\left(k_{i} \cdot Y_{i}\right) A\left(1,\left.\boldsymbol{\sigma} \in\left(\boldsymbol{\zeta}^{(1)} \Psi^{\prime} \boldsymbol{\xi}\right)\right|_{a_{1} \prec a}, r\right)=-\mathcal{B}_{G}^{a_{1}}\left(1\left|\mathcal{T}_{a}^{1}, \boldsymbol{\xi}\right| r\right)
$$

Thus the sum of all contributions from $\mathcal{T}_{a}^{1}$ in eq. (7.29) reads (recall that $f^{a_{1}}=-f^{a}=-1$ )

$$
-\sum_{\left.\boldsymbol{\zeta}^{(2)} \in \mathcal{T}_{a}^{2}\right|_{a_{2}}} \sum_{\left.\zeta^{(3)} \in \mathcal{T}_{a}^{3}\right|_{a_{3}}} \sum_{\xi \in\left\{a, \zeta^{(2)} \uplus \zeta^{(3)}\right\} \uplus \gamma} \mathcal{B}_{G}^{a_{1}}\left(1\left|\mathcal{T}_{a}^{1}, \boldsymbol{\xi}\right| r\right) .
$$

Following similar discussions, the sum of contributions from $\mathcal{T}_{a}^{2}$ and $\mathcal{T}_{a}^{3}$ are respectively given by the replacements of labels $1 \leftrightarrow 2$ and $1 \leftrightarrow 3$. The expression eq. (7.28) is then expressed by terms with respect to subtree structures

$$
\begin{aligned}
& \sum_{\boldsymbol{\sigma}} \sum_{\left.\boldsymbol{\zeta} \in \mathcal{T}\right|_{a}}\left(k_{a} \cdot Y_{a}\right) A(1, \boldsymbol{\sigma} \in(\boldsymbol{\zeta} \amalg \boldsymbol{\gamma}), r)-\left[\sum_{\left.\boldsymbol{\zeta}^{(2)} \in \mathcal{T}_{a}^{2}\right|_{a_{2}}} \sum_{\left.\boldsymbol{\zeta}^{(3)} \in \mathcal{T}_{a}^{3}\right|_{a_{3}}} \sum_{\boldsymbol{\xi} \in\left\{a, \boldsymbol{\zeta}^{(2)} \uplus \boldsymbol{\zeta}^{(3)}\right\} \amalg \boldsymbol{\gamma}} \mathcal{B}_{G}^{a_{1}}\left(1\left|\mathcal{T}_{a}^{1}, \boldsymbol{\xi}\right| r\right)\right. \\
& +\sum_{\boldsymbol{\zeta}^{(1)} \in \mathcal{T}_{a}^{1} \mid a_{1}} \sum_{\left.\boldsymbol{\zeta}^{(3)} \in \mathcal{T}_{a}^{3}\right|_{a_{3}}} \sum_{\boldsymbol{\xi} \in\left\{a, \boldsymbol{\zeta}^{(1)} \uplus \boldsymbol{\zeta}^{(3)}\right\} \uplus \boldsymbol{\gamma}} \mathcal{B}_{G}^{a_{2}}\left(1\left|\mathcal{T}_{a}^{2}, \boldsymbol{\xi}\right| r\right) \\
& \left.+\sum_{\left.\boldsymbol{\zeta}^{(2)} \in \mathcal{T}_{a}^{2}\right|_{a_{2}}} \sum_{\left.\boldsymbol{\zeta}^{(1)} \in \mathcal{T}_{a}^{1}\right|_{a_{1}}} \sum_{\boldsymbol{\xi} \in\left\{a, \boldsymbol{\zeta}^{(2)} \uplus \boldsymbol{\zeta}^{(1)}\right\} \uplus \gamma} \mathcal{B}_{G}^{a_{3}}\left(1\left|\mathcal{T}_{a}^{3}, \boldsymbol{\xi}\right| r\right)\right] .
\end{aligned}
$$

The above discussion is naturally generalized to arbitrary tree structure $\mathcal{T}$. For any $\mathcal{B}_{G}^{a}$, we have

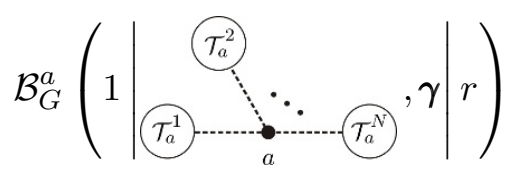

$$
\begin{aligned}
& =\sum_{\boldsymbol{\sigma}} \sum_{\left.\boldsymbol{\zeta} \in \mathcal{T}\right|_{a}}\left(k_{a} \cdot Y_{a}\right) A(1, \boldsymbol{\sigma} \in(\boldsymbol{\zeta} \amalg \boldsymbol{\gamma}), r) \\
& -\left[\left.\sum_{\substack{\left.\zeta^{(j)} \in \mathcal{T}_{a}^{j}\right|_{a_{j}} \\
\text { for } j=2, \ldots, N}} \sum_{ш} \mathcal{B}_{G}^{a_{1}}\left(1\left|\mathcal{T}_{a}^{1},\left\{a, \zeta^{(1)} ш \boldsymbol{\zeta}^{(2)} ш \cdots \uplus \boldsymbol{\zeta}^{(N)}\right\} ш \gamma\right| r\right)\right|_{a_{1} \prec a}\right. \\
& +\left.\sum_{\substack{\left.\boldsymbol{\zeta}^{(j)} \in \mathcal{T}_{a}^{j}\right|_{a_{j}} \\
\text { for } j=1,3, \ldots, N}} \sum_{ш} \mathcal{B}_{G}^{a_{2}}\left(1\left|\mathcal{T}_{a}^{2},\left\{a, \boldsymbol{\zeta}^{(1)} ш \zeta^{(2)} ш \cdots ш \boldsymbol{\zeta}^{(N)}\right\} ш \gamma\right| r\right)\right|_{a_{2} \prec a} \\
& \left.+\ldots+\left.\sum_{\substack{\left.\boldsymbol{\zeta}^{(j)} \in \mathcal{T}_{a}^{j}\right|_{a_{j}} \\
\text { for } j=1, \ldots, N-1}} \sum_{ш} \mathcal{B}_{G}^{a_{N}}\left(1\left|\mathcal{T}_{a}^{N},\left\{a, \boldsymbol{\zeta}^{(1)} ш \boldsymbol{\zeta}^{(2)} ш \cdots ш \boldsymbol{\zeta}^{(N)}\right\} ш \boldsymbol{\gamma}\right| r\right)\right|_{a_{N} \prec a}\right],
\end{aligned}
$$

where we wrote the sum over multi-shuffle permutations by $\sum_{\Psi}$ for short. According to the inductive assumption, each term in the square brackets for a given $i$ can be expressed by 
the sum of the following two terms:

$$
\left.\sum_{\substack{\left.\boldsymbol{\zeta}^{(j)} \in \mathcal{T}_{a}^{j}\right|_{a_{j}} \\ \text { for } j=1, \ldots, N, j \neq i}} \sum_{\boldsymbol{\xi}^{(i)}} \mathcal{B}_{G}^{(1)}\left(1\left|\mathcal{T}_{a}^{i}\right|_{a_{i}}, \boldsymbol{\xi}^{(i)} \mid r\right)\right|_{a_{i} \prec a}=\sum_{\substack{\left.\boldsymbol{\zeta}^{(j)} \in \mathcal{T}_{a}^{j}\right|_{a_{j}} \\ \text { for } j=1, \ldots, N}} \sum_{\boldsymbol{\xi}^{(i)}}\left[\mathcal{B}\left(1\left|\boldsymbol{\zeta}^{(i)}, \boldsymbol{\xi}^{(i)}\right| r\right)-\left.\mathcal{B}\left(1\left|\boldsymbol{\zeta}^{(i)}, \boldsymbol{\xi}^{(i)}\right| r\right)\right|_{a_{i} \succ a}\right]
$$

and

$$
\sum_{\substack{\left.\boldsymbol{\zeta}^{(j)} \in \mathcal{T}_{a}^{j}\right|_{a_{j}} \\ \text { for } j=1, \ldots, N, j \neq i}} \sum_{\boldsymbol{\xi}^{(i)}}\left[\left.\sum_{\amalg} \mathcal{B}_{G}^{(2)}\left(1\left|\mathcal{T}_{a}^{i}-a_{i},\left\{a_{i}\right\} ш \boldsymbol{\xi}^{(i)}\right| r\right)\right|_{a_{i} \prec a}\right]
$$

In eq. (7.35) and eq. (7.36), the summations over $\boldsymbol{\xi}^{(i)}$ are taken by summing over all

$$
\boldsymbol{\xi}^{(i)} \in\left\{\left\{a, \boldsymbol{\zeta}^{(1)} ш \cdots ш \zeta^{(i)} ш \cdots ш \boldsymbol{\zeta}^{(N)}\right\} ш \boldsymbol{\gamma}\right\},
$$

Eq. (7.34) is then given by summing the following two contributions together

- The sum of all the last terms on the r.h.s. of eq. (7.35) for all $i=1, \ldots, N$ and the first term of eq. (7.34). According to eq. (2.11), the last term in the brackets of eq. (7.35) for given $\left.\boldsymbol{\zeta}^{(j)} \in \mathcal{T}_{a}^{j}\right|_{a_{j}}(j=1,2, \ldots, N)$ when summing over $\boldsymbol{\xi}^{(i)}$ can be explicitly written as

$$
\left.\sum_{\boldsymbol{\xi}^{(i)}} \mathcal{B}\left(1\left|\boldsymbol{\zeta}^{(i)}, \boldsymbol{\xi}^{(i)}\right| r\right)\right|_{a_{i} \succ a}=\sum_{\boldsymbol{\xi}^{(i)}} \sum_{\boldsymbol{\eta} \in \boldsymbol{\zeta}^{(i)} \uplus \boldsymbol{\xi}^{(i)} \mid a_{i} \succ a} \sum_{x \in \boldsymbol{\zeta}^{(i)}}\left(k_{x} \cdot X_{x}(\boldsymbol{\eta})\right) A(1, \boldsymbol{\eta}, r) .
$$

Here, $\sum_{\boldsymbol{\xi}^{(i)}} \sum_{\left.\boldsymbol{\eta} \in \boldsymbol{\zeta}^{(i)} \uplus \boldsymbol{\xi}^{(i)}\right|_{a_{i} \succ a}}$ is given by summing over permutations

$$
\left.\left(\zeta^{(i)} \uplus\left\{\left\{a, \zeta^{(1)} ш \cdots ш \zeta^{(i)} \uplus \cdots ш \zeta^{(N)}\right\} ш \gamma\right\}\right)\right|_{a_{i} \succ a} .
$$

Recalling that $a_{i}$ is the leftmost element in $\left.\boldsymbol{\zeta}^{(i)} \in \mathcal{T}_{a}^{i}\right|_{a_{i}}$, the above expression turns to

$$
\left\{a, \zeta^{(1)} ш \cdots ш \boldsymbol{\zeta}^{(i)} ш \cdots ш \boldsymbol{\zeta}^{(N)}\right\} ш \boldsymbol{\gamma} .
$$

Therefore, the sum of all the last terms of in eq. (7.35) over all $i=1, \ldots, N$ and the first term of eq. (7.34) gives

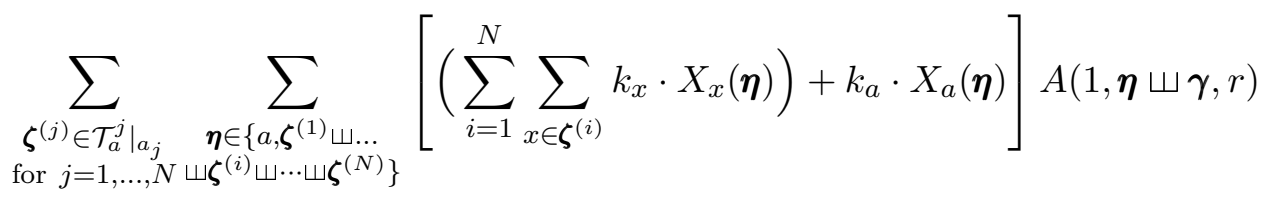

$$
\begin{aligned}
& =\sum_{\substack{\left.\boldsymbol{\zeta}^{(j)} \in \mathcal{T}_{a}^{j}\right|_{a_{j}} \\
\text { for } j=1, \ldots, N}} \sum_{ш} \mathcal{B}\left(1\left|\left\{a, \boldsymbol{\zeta}^{(1)} ш \cdots ш \boldsymbol{\zeta}^{(N)}\right\}, \gamma\right| r\right)
\end{aligned}
$$

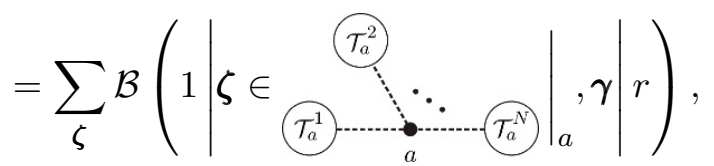

which is just $\mathcal{B}_{G}^{(1)}$ in eq. (7.26). 
- The sum of the first term in eq. (7.35) and eq. (7.36) over all $i=1, \ldots, N$. This part defines

$$
\begin{aligned}
& \sum_{ш} \mathcal{B}_{G}^{(2)}\left(1\left|\widetilde{\mathcal{T}_{a}^{1}} \widetilde{\mathcal{T}_{a}^{2}} \cdots \widetilde{\mathcal{T}_{a}^{N}},\{a\} ш \gamma\right| r\right) \\
& =\sum_{i=1}^{N} \sum_{\substack{\left.\boldsymbol{\zeta}^{(j)} \in \mathcal{T}_{a}^{j}\right|_{a_{j}} \\
\text { for } j=1, \ldots, N, j \neq i}} \sum_{\boldsymbol{\xi}^{(i)}}\left[\sum_{\boldsymbol{\zeta}^{(i)}} \mathcal{B}\left(1\left|\boldsymbol{\zeta}^{(i)} \in \mathcal{T}_{a}^{i}\right|_{a_{i}}, \boldsymbol{\xi}^{(i)} \mid r\right)\right. \\
& \left.+\left.\sum_{\varpi} \mathcal{B}_{G}^{(2)}\left(1\left|\mathcal{T}_{a}^{i}-a_{i},\left\{a_{i}\right\} ш \boldsymbol{\xi}^{(i)}\right| r\right)\right|_{a_{i} \prec a}\right],
\end{aligned}
$$

where $\boldsymbol{\xi}^{(i)}$ is summed over all permutations satisfying eq. (7.37). In the square brackets, the first term is precisely the l.h.s. of BCJ relation eq. (2.10) where $a$ is in the $\boldsymbol{\alpha}$ set. According to the inductive assumption, the second term is a combination of BCJ relations where both $a_{i}$ is in the $\boldsymbol{\alpha}$ set. Since $a \in \boldsymbol{\xi}^{(i)}, a$ must also be in the $\boldsymbol{\alpha}$ set. Thus the second term of eq. (7.42) is a combination of the 1.h.s. of those BCJ relations with $a_{i} \prec a$.

All together, we have proven that the l.h.s. of the graph-based BCJ relation is a combination of the l.h.s. of traditional BCJ relations.

\section{Gauge invariance identities of tree-level YM and GR amplitudes}

A tree level $n$ graviton amplitude $M(1,2, \ldots, n)$ can be expanded as a combination of tree level single-trace EYM amplitudes. Particularly, we can pick out arbitrary two gravitons, for example, 1 and $n$ and then write the amplitude $M(1,2, \ldots, n)$ in terms of single-trace EYM amplitudes, where 1 and $n$ are always considered as two gluons, as follows

$$
M(1,2, \ldots, n)=\sum_{\text {Splits }} \sum_{\boldsymbol{\sigma} \in \text { perms A }}(-1)^{|\mathrm{A}|}\left(\epsilon_{1} \cdot F_{\sigma(1)} \cdots F_{\sigma(|\mathrm{A}|)} \cdot \epsilon_{n}\right) A(1, \boldsymbol{\sigma}, n \| \mathbf{H}) .
$$

Here, $|\mathrm{A}|$ is the number of elements in $\mathrm{A}$. We have summed over all splits $\{2, \ldots, n-1\} \rightarrow$ $\mathrm{A}, \mathrm{H}$ in the first summation. For a given A, we summed over all possible permutations $\boldsymbol{\sigma}$ of elements in A. When the EYM amplitudes in eq. (8.1) are expressed by eq. (2.4), eq. (8.1) is then expanded in terms of pure YM amplitudes

$$
M(1,2, \ldots, n)=\sum_{\text {Splits }} \sum_{\boldsymbol{\sigma} \in \text { perms A }} \sum_{\boldsymbol{\xi} \in \boldsymbol{\sigma} \amalg \text { perms } \mathrm{H}} W(1, \boldsymbol{\sigma}, n) \mathcal{C}(1, \boldsymbol{\xi}, n) A(1, \boldsymbol{\xi}, n),
$$

where

$$
W(1, \sigma, n) \equiv \epsilon_{n} \cdot F_{\sigma(|\mathrm{A}|)} \cdots F_{\sigma(1)} \cdot \epsilon_{1} .
$$

The factor $(-1)^{|\mathrm{A}|}$ in eq. (8.1) is absorbed when the antisymmetry of the strength tensors are considered. If we collect all coefficients $n_{1|\zeta| n}$ corresponding to a given permutation $\boldsymbol{\zeta}$ of elements in $\{2, \ldots, n-1\}$ together, a briefer form (dual DDM form) is obtained

$$
M(1,2, \ldots, n)=\sum_{\boldsymbol{\zeta} \in S_{n-2}} n_{1|\boldsymbol{\zeta}| n} A(1, \boldsymbol{\zeta}, n),
$$

where $n_{1|\zeta| n}$ are the BCJ numerators corresponding to half-ladder diagrams. 
The gauge invariance condition for any graviton $a$ states that the GR amplitude $M(1,2, \ldots, n)$ must vanish under the replacement $\epsilon_{a} \rightarrow k_{a}$. If the graviton $a$ belongs to $\{2, \ldots, n-1\}$, it can be an element of either $\mathrm{A}$ or $\mathrm{H}$ for a given split in eq. (8.1). For the latter case, $a$ is considered as a graviton in an EYM amplitude whose gauge invariance induced identity has been studied in the previous sections. For the former case, the gauge invariance is already included in the coefficients $W(1, \sigma, n)$ due to the antisymmetry of the strength tensors. Therefore, only the identity induced by the gauge invariance condition for $a=1$ or $n$ requires further study.

In the following, we review the old-version graphic rule for the construction of BCJ numerators $n_{1|\zeta| n}$ in eq. (8.4). Then we expand these graphs by refined graphs and prove that the gauge invariance induced identity

$$
\left.\sum_{\zeta \in S_{n-2}} n_{1|\zeta| n}\right|_{\epsilon_{1} \rightarrow k_{1}} A(1, \zeta, n)=0
$$

is a combination of BCJ relations. The identity induced by the condition $\epsilon_{n} \rightarrow k_{n}$ can be studied in a similar way. Once all GR, EYM and YM amplitudes are replaced by YM, YMS (Yang-Mills scalar) and BS (biscalar) amplitudes correspondingly, the discussions are immediately extended to the identities (for BS amplitudes) induced by the gauge invariance of YM amplitudes.

\subsection{The old-version graphic rule for the BCJ numerators $n_{1|\zeta| n}$}

BCJ numerators $n_{1|\boldsymbol{\zeta}| n}$ obtained from eq. (8.2) can be constructed according to the following (old version) graphic rule [12]: ${ }^{9}$

Step-1. Define a reference order of elements $2,3, \ldots, n-1, n$ by $\mathrm{R}=\{\rho(2), \rho(3), \ldots$, $\rho(n-1), n\}$ where the graviton $n$ is considered as the last element, $\boldsymbol{\rho}$ is a permutation of $2,3, \ldots, n-1$. Define weight of elements $2,3, \ldots, n-1, n$ by their positions in $\mathrm{R}$.

Step-2. For a given permutation $\boldsymbol{\zeta}$, pick out $i_{1}, i_{2}, \ldots, i_{j}$ and $n$ from $\mathrm{R}=\{\rho(2), \rho(3), \ldots$, $\rho(n-1), n\}$ such that the following condition is satisfied

$$
\zeta^{-1}(1)<\zeta^{-1}\left(i_{1}\right)<\cdots<\zeta^{-1}\left(i_{j}\right)<\zeta^{-1}(n)
$$

Then we construct a chain $\mathbb{C H}=\left[n, i_{j}, i_{j-1}, \ldots, i_{1}, 1\right]$ which starts from the node $n$, passes through internal nodes $i_{j}, \ldots, i_{1}$ and ended at 1 . The node 1 is considered as the root. The factor corresponding to the chain $\mathbb{C H} I=\left[n, i_{j}, i_{j-1}, \ldots, i_{1}, 1\right]$ is

$$
W\left(1, i_{1}, \ldots, i_{j}, n\right)=\epsilon_{n} \cdot F_{i_{j}} \cdots F_{i_{1}} \cdot \epsilon_{1} .
$$

Redefine the reference order by $\mathrm{R} \rightarrow \mathrm{R}^{\prime}=\mathrm{R} \backslash\left\{i_{1}, \ldots, i_{j}, n\right\} \equiv\left\{\rho^{\prime}(1), \ldots, \rho^{\prime}\left(n^{\prime}\right)\right\}$.

\footnotetext{
${ }^{9}$ Although the interpretation of the rule in this paper is different from the version in [12], they essentially provide the same construction.
} 
Step-3. Pick out $\rho^{\prime}\left(n^{\prime}\right), i_{1}^{\prime}, \ldots, i_{j^{\prime}}^{\prime}$ from $\mathrm{R}^{\prime}$. Construct a chain $\mathbb{C H}=\left[\rho^{\prime}\left(n^{\prime}\right), i_{j^{\prime}}^{\prime}, i_{j^{\prime}-1}^{\prime}, \ldots\right.$, $\left.i_{1}^{\prime}, a\right]$ towards $a \in\{1\} \cup\left\{i_{1}, i_{2}, \ldots, i_{j}\right\}$ (noting that $a$ cannot be $n$ because $n$ is always considered as the last element) such that

$$
\zeta^{-1}(a)<\zeta^{-1}\left(i_{1}^{\prime}\right)<\cdots<\zeta^{-1}\left(i_{j^{\prime}}^{\prime}\right)<\zeta^{-1}\left(\rho^{\prime}\left(n^{\prime}\right)\right)
$$

is satisfied. This chain corresponds to a factor

$$
\epsilon_{\rho^{\prime}\left(n^{\prime}\right)} \cdot F_{i_{j^{\prime}}^{\prime}} \cdot \ldots F_{i_{1}^{\prime}} \cdot k_{a}
$$

Redefine the ordered set by $\mathrm{R} \rightarrow \mathrm{R}^{\prime \prime}=\mathrm{R}^{\prime} \backslash\left\{i_{1}^{\prime}, \ldots, i_{j^{\prime}}^{\prime}, \rho^{\prime}\left(n^{\prime}\right)\right\} \equiv\left\{\rho^{\prime \prime}(1), \ldots, \rho^{\prime \prime}\left(n^{\prime \prime}\right)\right\}$.

Step-4. Repeat the above step. In each step, we define a chain which starts from the highest-weight element in the new defined $\mathrm{R}$ and ends at any node on chains constructed previously except the node $n$ (the root 1 should be included). Redefine $\mathrm{R}$ by removing the starting and internal nodes which have been used in the current step. This process is terminated when $\mathrm{R}$ is empty. All chains together form a connected tree graph with the root 1. Multiplying all factors corresponding to the chains and summing over all possible graphs for the permutation $\boldsymbol{\zeta}$, we finally get the BCJ numerator $n_{1|\zeta| n}$.

\subsection{Understanding the gauge invariance induced identity eq. (8.5)}

When the condition $\epsilon_{1} \rightarrow k_{1}$ is imposed and all strength tensors $F_{i}^{\mu \nu}$ are expanded explicitly according to the definition eq. (2.3), the three types of chains (see figure 3) are distinguished. Hence the old-version graphic rule becomes a refined rule. The coefficients $\left.n_{1|\zeta| n}\right|_{\epsilon_{1} \rightarrow k_{1}}$ in the identity eq. (8.5) is then provided by

$$
\left.n_{1|\zeta| n}\right|_{\epsilon_{1} \rightarrow k_{1}}=\sum_{\mathcal{F} \in \widetilde{G}(1, \zeta, n)} \widetilde{\mathcal{D}}^{[\mathcal{F}]}
$$

where $\widetilde{\mathbb{G}}(1, \zeta, n)$ is the set of physical graphs defined by the refined graphic rule and we summed over all possible graphs $\mathcal{F} \in \widetilde{\mathbb{G}}(1, \zeta, n)$. The factor $\widetilde{\mathcal{D}}^{[\mathcal{F}]}$ denotes the coefficient for a given graph constructed by the refined rule. The identity eq. (8.5) then turns to

$$
\sum_{\boldsymbol{\zeta} \in S_{n-2}}\left[\sum_{\mathcal{F} \in \widetilde{\mathbb{G}}(1, \boldsymbol{\zeta}, n)} \widetilde{\mathcal{D}}^{[\mathcal{F}]}\right] A(1, \boldsymbol{\zeta}, n)=0 .
$$

Before showing the relationship between the identity eq. (8.11) and BCJ relation eq. (2.10), we point out the following features of graphs $\mathcal{F}$ in eq. (8.11):

(i) For any permutation $1, \boldsymbol{\zeta}, n$, the nodes 1 and $n$ are always the leftmost and the rightmost nodes respectively. The node 1 always plays as the root of a graph $\mathcal{F}$. The node $n$ cannot be the end node of other chains.

(ii) Since $\epsilon_{1}$ is replaced by $k_{1}$, the node 1 must be connected by lines with arrows pointing to it. Hence only type-2 lines (with the arrows pointing to 1 ) and type- 3 lines can be connected to 1 . 
(iii) Any starting node of a chain is associated with a polarization, thus each of them starts a type- 1 line or a type-2 line pointing towards the direction of the root.

(iv) Being different from the graphs for the identity induced from EYM amplitudes, graphs that do not involve any type-3 line are allowed. In such a graph, all lines are type-2 lines pointing to the direction of the root 1 .

To study eq. (8.11), we classify all graphs into two categories according to wether the node $n$ is connected by a type- 1 line or a type-2 line. Then the l.h.s. of eq. (8.11) is written as the sum of $T_{1}$ and $T_{2}$ :

$$
T_{1} \equiv \sum_{\boldsymbol{\zeta} \in S_{n-2}}\left[\sum_{\mathcal{F} \in \widetilde{\mathbb{G}}_{1}(1, \boldsymbol{\zeta}, n)} \widetilde{\mathcal{D}}^{[\mathcal{F}]}\right] A(1, \boldsymbol{\zeta}, n), \quad T_{2} \equiv \sum_{\boldsymbol{\zeta} \in S_{n-2}}\left[\sum_{\mathcal{F} \in \widetilde{\mathbb{G}}_{2}(1, \boldsymbol{\zeta}, n)} \widetilde{\mathcal{D}}^{[\mathcal{F}]}\right] A(1, \boldsymbol{\zeta}, n),
$$

where $\widetilde{\mathbb{G}}_{1}(1, \boldsymbol{\zeta}, n)$ and $\widetilde{\mathbb{G}}_{2}(1, \boldsymbol{\zeta}, n)$ denote the sets of graphs where $n$ is connected by a type-1 line and a type-2 line (pointing to the direction of root), respectively. We study these two parts separately.

The $\boldsymbol{T}_{\mathbf{1}}$ part. If the node $n$ is connected by a type- 1 line, the chain $\mathbb{C H}=\left[n, i_{j}, i_{j-1}, \ldots\right.$, $i_{1}, 1$ ] contributing to $T_{1}$ (see eq. (8.12)) can be described by figure 9 (b) when replacing the nodes $b$ and $h_{\rho(s)}$ therein respectively by the node 1 and the node $i_{j}$ that is further connected by a type- 1 line starting at the node $n$. According to discussions in section 5 , such a chain must involve at least one type-3 line. As a result, the skeleton (the graph obtained by removing all type-3 lines) for a graph contributing to $T_{1}$ must be a disconnected graph. Following a similar discussion with section 5, we find that there are three types of components corresponding to a given skeleton in general (as in the EYM case):

- Type-I component: A component which contains neither 1 nor $n$ and involves a type-1 line (see figure 19 (a)). In general, such a component can have type-2 lines whose arrows point to the direction of the ends of type-1 lines (i.e. the kernel).

- Type-II component: A component containing the node $n$ (see figure 19 (b)). Components of this type also have a kernel: the type-1 line with two ends $n$ and $a(a \neq n, 1)$. The difference from type-I components is that all possible type- 2 lines must be only on the bottom side (i.e. the side containing node $a$ ) of this component. The node $n$ is the starting node of the kernel (the type- 1 line) of this component, while it cannot be the ending node of any chain. Thus there cannot be lines ended at $n$.

- Type-III component: A component containing the root 1 (see figure 19 (c)). Such a component only involve type-2 lines whose arrows point to the direction of the root 1.

A typical skeleton for graphs contributing to $T_{1}$ with $n=5$ is presented by figure 20 (a).

Having defined these three types of components, we can follow all the discussions in section 6 and appendix D. The only thing we need to pay more attention to is that the node 


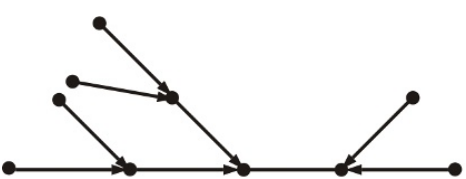

(a)

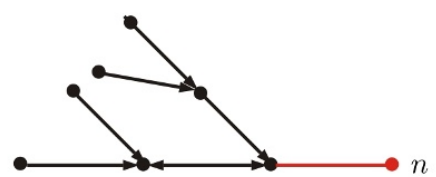

(b)

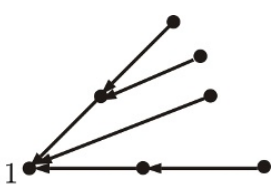

(c)

Figure 19. Graphs (a), (b) and (c) are correspondingly typical graphs of type-I, -II,-III components in the skeleton of the $T_{1}$ part in eq. (8.12).

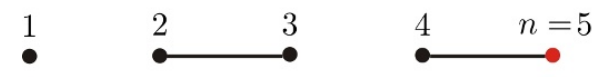

(a)

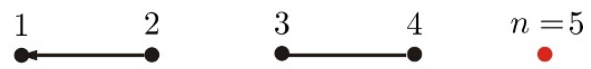

(b)

Figure 20. Graphs (a) and (b) are typical skeletons (with three components) for graphs contributing to $T_{1}$ and $T_{2}$ (see eq. (8.12)) with $n=5$, respectively. Here the reference order is chosen as $\mathrm{R}=\{1,2,3,4,5\}$. In graph (a), the type- 1 line with its two ends 2 and 3 , the type- 1 line with its two ends 4 and 5, the single node $a$ are respectively type-I, -II and -III components. In graph (b), the type- 2 line with its two ends 1 and 2 (the arrow points to 1 ), the type- 1 line with its ends 3 and 4 , the single node 5 are respectively type-I, -II and -III components.

$n$ in the type-II component cannot be connected with nodes in other components via type3 lines and it must be the rightmost element in any permutation. For any configuration of final upper and lower blocks, the sum of all physical graphs and spurious graphs is proportional to the 1.h.s. of the graph-based BCJ relation. For example, the sum of graphs figure 21 (a) and (b) provides $(-1)^{2}\left(\epsilon_{2} \cdot \epsilon_{3}\right)\left(\epsilon_{4} \cdot \epsilon_{5}\right)\left(k_{1} \cdot k_{2}\right) \mathcal{B}_{G}^{4}\left(1||_{\bullet}^{4},\{2,3\} \mid 5\right)$ while the sum of graphs (c) and (d) contributes $\left(\epsilon_{2} \cdot \epsilon_{3}\right)\left(\epsilon_{4} \cdot \epsilon_{5}\right)\left(k_{2} \cdot k_{4}\right) \mathcal{B}_{G}^{3}\left(\left.1\right|^{3} \ldots \ldots \ldots \ldots \ldots\right.$ we conclude that the $T_{1}$ part can always be written as a combination of the l.h.s. of graphbased BCJ relations eq. (7.1) which have been proven to be a combination of traditional BCJ relations eq. (2.10).

The $\boldsymbol{T}_{\mathbf{2}}$ part. Graphs contributing to $T_{2}$ involve the node $n$ which is connected by a type-2 line (pointing towards the direction of root). To expand $T_{2}$ by BCJ relations, we should adjust the definition of skeleton by those graphs which are obtained by removing both type-3 lines and the type-2 line attached to the node $n$. Any skeleton under this definition is a disconnected graph. The definition of type-I and type-III components are same with those in the $T_{1}$ case. The type-II component is defined by the single node $n$. Along the line pointed in section 6 , we define the upper and lower blocks by the maximally connected graphs containing the node $n$ and 1 respectively. At the beginning, the upper and lower blocks are correspondingly the type-II and the type-III components. According to disucssions in section 6.2, we attach a chain of components to either the upper or the lower block and redefine these blocks in each step. In this case, we should require that the node $n$ can only be connected by the first chain that is attached to the upper block, via a type-2 line (with the arrows pointing towards root). When all components are used, we 


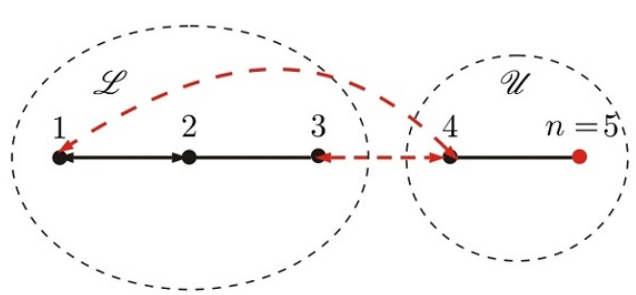

(a)

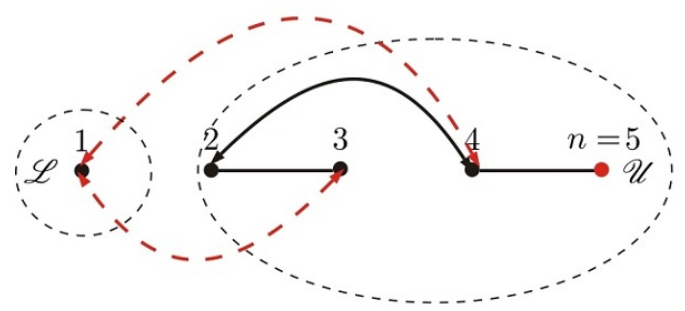

(c) (b)
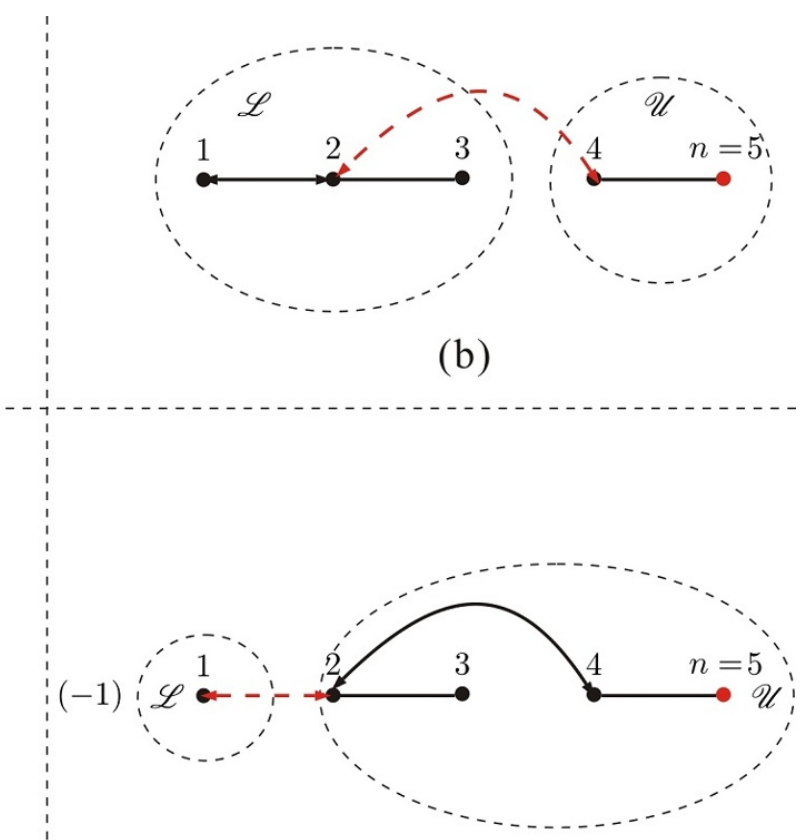

(d)

Figure 21. Graphs (a) and (c) are physical graphs corresponding to the skeleton figure 20 (a). They are obtained by constructing a chain of components, which starts from the type-I component, towards the lower and upper blocks respectively according to discussions in section 6 (noting that the node $n$ cannot be the ending node of any chain). Thus there are two distinct configurations of the final upper and lower blocks $\mathscr{U}$ and $\mathscr{L}$. Spurious graphs are given by (b) and (d). Since the spurious component, whose only a single side (i.e. the node 2) on the part from the highest weight node 5 to the root 1 , belongs to the final upper block in (d), we should have an extra minus. Apparently graphs (b) and (d) cancel with each other.

arrive the final upper and lower blocks. As done in section 6, we connect these two blocks into a fully connected graph:

(i) If the final upper block consists of more than one node, such as figure 22 (a), (b), we should connect a node $b$ in the final lower block with a node $a(b \neq n)$ in the final upper block via a type-3 line.

(ii) If the final upper block only contains the node $n$, such as figure 22 (c), (d), (e) and (f), we should connect a node $b$ in the final lower block with $n$ via a type-2 line whose arrow points to $a$.

After this step, a graph is a spurious one (for example figure 22 (b), (d) and (f)) if it contains spurious components (whose only a single side is passed through by the path from $n$ to 1 ), otherwise, it is a physical graph (for example figure 22 (a), (c) and (e)). The extra sign associated with a spurious graph is given by $(-1)^{\mathcal{S}(\mathscr{U})}$, where $\mathcal{S}(\mathscr{U})$ is the number of spurious components in the final upper block. Spurious graphs belonging to distinct final upper and lower blocks cancel in pairs as shown in section 6.2. For example the two graphs included by figure 22 (b) cancel with figure 22 (d) and (e), respectively. Hence, the $T_{2}$ 


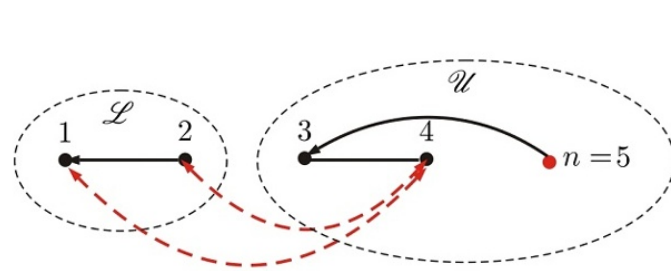

(a)

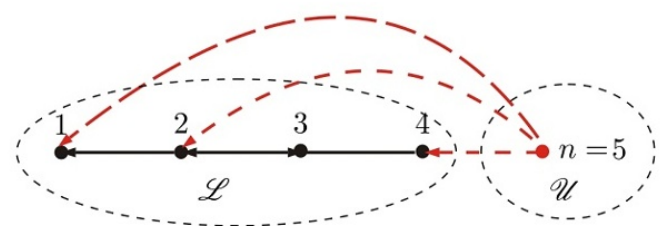

(c)

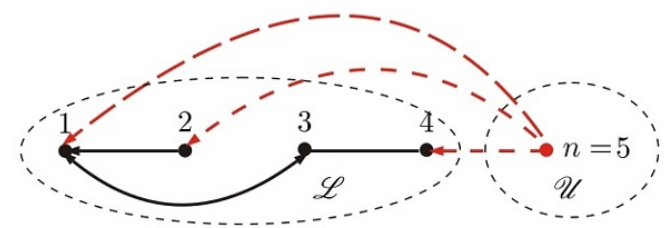

(e)

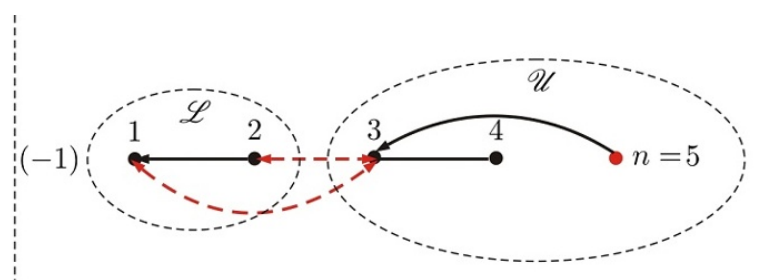

(b)

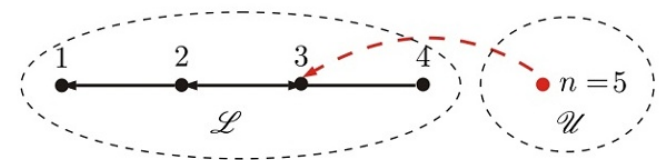

(d)

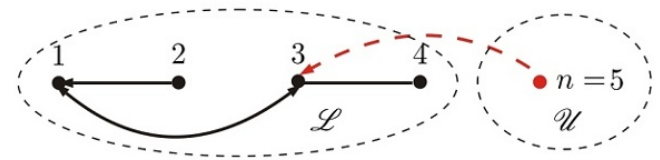

(f)

Figure 22. Graphs (a), (c) and (e) are physical graphs corresponding to the skeleton figure 20 (b). The type-I chain belongs to the final upper (lower) block in (a)((c)and (e)). Corresponding spurious graphs are given by (b), (d) and (f). The final upper block in (b) contains a spurious component (i.e. the type-I component), thus there should be an extra minus. In graphs (d) and (f), the final upper block does not contain any spurious component, thus the sign is plus. All spurious graphs (b), (d) and (f) cancel out (noting that (b) stands for two graphs).

can be given by summing over (1) all possible configurations of the final upper and lower blocks, (2) all possible graphs constructed by connecting these two blocks according to (i) and (ii) pointed above for a given configuration. Particularly,

(1) If the final upper block consists of more than one node, the sum of all graphs given by (i) is expanded in terms of the l.h.s. of the graph-based BCJ relations eq. (7.1) (hence the 1.h.s. of traditional BCJ relations eq. (2.10)). For example the sum of graphs included in figure 22 (a) and (b) is proportional to the l.h.s. of a graph-based BCJ relation $(-1)\left(\epsilon_{1} \cdot \epsilon_{2}\right)\left(\epsilon_{3} \cdot \epsilon_{4}\right)\left(\epsilon_{5} \cdot k_{3}\right) B_{G}^{4}\left(\left.1\right|_{\bullet \cdots \cdots-\cdots} ^{3}{ }^{4},\{2\} \mid 5\right)$.

(2) If the final upper block consists of only the node $n, n$ can be connected to any node $b$ in the final lower block via a type-2 line $\epsilon_{n} \cdot k_{a}$ (see figure $22(\mathrm{c}),(\mathrm{d})$ and (e), (f) for distinct configurations of the final lower block). The permutations established by graphs with all choices of $b$ are same. Thus we can extract a total factor

$$
\sum_{a \in\{1,2, \ldots, n-1\}} \epsilon_{n} \cdot k_{a}=\epsilon_{n} \cdot\left(-k_{n}\right)=0
$$

where the transversality of polarization and momentum conservation have been applied. Hence this part must vanish. 
To sum up, the identity eq. (8.5) which is induced by the gauge invariance of gravity can always been expressed as a combination of BCJ relations.

\section{Conclusions and further discussions}

In this paper, a graphic approach to the relationship between gauge invariance induced identity (where coefficients are polynomials of Lorentz inner products $\epsilon \cdot \epsilon, \epsilon \cdot k$ and $k \cdot k$ ) and BCJ relations (where coefficients are functions of only Mandelstam variables $k \cdot k$ ) is provided. By establishing a refined graphic rule, the three types of Lorentz inner products $\epsilon \cdot \epsilon, \epsilon \cdot k$ and $k \cdot k$ are represented by type-1, -2 and -3 lines correspondingly. To find out the relationship between the identity induced from the gauge invariance of tree level single-trace EYM amplitude and BCJ relations, we collected terms containing the same skeleton (the subgraph obtained by deleting all type-3 lines). We further proved that the sum of physical graphs for a given skeleton can be given by (a) summing over all possible configurations of final upper and lower blocks; (b) for a given configuration, summing over all possible (physical and spurious) graphs constructed by connecting a type-3 line between the final upper and lower blocks. Each summation in (b) is in fact proportional to a graph-based BCJ relation which was further proved to be a combination of traditional BCJ relations. Following a similar discussion, we proved that the identity induced by the gauge invariance of tree level GR (YM) amplitudes could also be expanded in terms of traditional BCJ relations.

Although, the gauge invariance identity and standard BCJ relations have quite different forms and origins, they can be related when an amplitude is written as the double copy form. As pointed in [25], BCJ relation among Yang-Mills amplitudes can be considered as a result of the Jacobi identities satisfied by BCJ numerators. On another hand, once we write a full color-dressed EYM amplitude or a GR amplitude into a double copy form, (according to discussions in [2]) the gauge invariance conditions for 'half polarizations' living in one copy numerators is ensured by the Jacobi identities for the other copy of numerators, which further result in the BCJ relations for color-ordered amplitudes. Thus the results in this paper can be regarded as a realization of the relationship between the gauge invariance and BCJ duality at amplitude level. We close this by pointing several interesting topics that deserve further study:

- First, how to extend the discussions to the identities induced from multi-trace EYM amplitudes? Two types of recursive expansions of tree level multi-trace EYM amplitudes were established in [17]. Correspondingly, there are two identities (mentioned as type-I and II in [17]) respectively induced by the gauge invariance condition of the fiducial graviton and the cyclic symmetry of a gluon trace. The type-II identity for amplitudes with two gluon traces and no graviton can be understood as the graphbased BCJ relation eq. (7.1) in which the tree graph $\mathcal{T}$ is a simple chain. However, this observation cannot be straightforwardly generalized to amplitudes with an arbitrary number of traces and gravitons. Thus the relationship between identities induced from arbitrary multi-trace EYM amplitudes still deserves further consideration. 
- Second, how to calculate helicity amplitudes in four dimensions by the refined graphic rule? In four dimensions, the tree level single-trace EYM and GR amplitudes with maximally-helicity-violating (MHV) configurations were shown to be proportional to (Hodges) determinants (see [33] for GR and [34] for EYM) which can be further expanded by graphs [34-36]. Such brief formulas cannot be trivially extended to arbitrary helicity configurations. It is worth studying the relationship between the graphic expansion of the Hodges determinants [33] and the refined graphic expansion of amplitudes in the current paper. This may provide hints for finding more simple formulas of helicity amplitudes in four dimensions.

- Third, it will be very interesting if one could find out the full relationship between the refined graphic rule and the expansion of CHY formula. Graphic expansions of CHY formula were already discussed in e.g., [30, 31, 37, 38]. In the work [31], a graphic expansion which is similar with the refined graphic rule has been proposed. It seems that there should be a very close relation between the expansion in [31] and the refined graphic rule in this paper.

- Last but not least, as shown in [26, 27], BCJ relation can be derived from monodromy of string amplitudes. It will be interesting if one can incorporate gauge invariance with string monodromy.

\section{Acknowledgments}

The authors are grateful to Gang Chen, Bo Feng, Chih-Hao Fu, Song He, Hui Luo, Fei Teng and Yong Zhang for helpful discussions or/and valuable comments. This work is supported by NSFC under Grant No. 11875206, Jiangsu Ministry of Science and Technology under contract BK20170410 as well as the "Fundamental Research Funds for the Central Universities".

\section{A Conventions and definitions}

$\mathrm{H}$ : the graviton set. $\quad F_{i}^{\mu \nu}$ : the strength tensor $k_{i}^{\mu} \epsilon_{i}^{\nu}-\epsilon_{i}^{\mu} k_{i}^{\nu}$

Permutations: $\boldsymbol{\alpha}, \boldsymbol{\beta}, \boldsymbol{\rho}, \boldsymbol{\sigma}, \boldsymbol{\xi}, \boldsymbol{\zeta} \quad \sigma(i)$ : the $i$-th element in $\boldsymbol{\sigma} \sigma^{-1}(l)$ : the position of $l$ in permutation $\sigma$

$A \varpi B$ for ordered sets $A$ and $B$ : permutations by merging $A$ and $B$ s.t. the relative order of elements in each set is kept, e.g. $\{1,2\} \amalg\{a, b\}=\{\{1,2, a, b\},\{1, a, 2, b\},\{1, a, b, 2\}$, $\{a, 1,2, b\},\{a, 1, b, 2\},\{a, b, 1,2\}\}$.

$Y_{i}(\boldsymbol{\sigma})$ for $\boldsymbol{\sigma} \in \boldsymbol{\alpha} \amalg \beta$ and $i \in \beta$ : the sum of all momenta of elements in $\boldsymbol{\sigma}$ (including the element 1) on the 1.h.s. of $i$ in permutation $\sigma$. 
$X_{i}(\boldsymbol{\sigma})$ for $\boldsymbol{\sigma} \in \boldsymbol{\alpha} ш \boldsymbol{\beta}$ and $i \in \beta$ : the sum of all momenta of elements in both $\beta$ and $\boldsymbol{\alpha}$ (including the element 1) that appear on the l.h.s. of $i$ in permutation $\sigma$.

$\mathcal{F}$ : Graphs. $\quad \mathrm{R}=\left\{h_{\rho(1)}, \ldots, h_{\rho(s)}\right\}$ : a reference order of gravitons $h_{1}, \ldots, h_{s}$

Weight of a graviton $h_{i}$ : the position of $h_{i}$ in the reference order $\mathrm{R}$

$\mathbb{C H}=\left[a, i_{j}, \ldots, i_{1}, b\right]$ : a chain of nodes with starting node $a$, ending node $b$ and internal nodes $i_{1}, \ldots, i_{j}$

$\mathcal{B}(1|\beta, \boldsymbol{\alpha}| r)$ : the l.h.s. of BCJ relation eq. (2.10)

$\mathcal{B}_{G}^{c}(1|\mathcal{T}, \gamma| r)$ : the l.h.s. of the graph based BCJ relation eq. (7.1) with choosing $f^{c}=1$

Type-1, 2 and 3 lines: lines corresponding to factors of forms $\epsilon_{a} \cdot \epsilon_{b}, \epsilon_{a} \cdot k_{b}$ and $k_{a} \cdot k_{b}$ in the refined graphic rule

$\mathcal{F}^{\prime} \subset \mathcal{F}$ : skeleton which is defined by removing all type-3 lines from a graph $\mathcal{F}$ for the relation

$\mathcal{F} \backslash \mathcal{F}^{\prime}$ for eq. (3.1): ${ }^{10}$ the subgraph of $\mathcal{F}$ which is obtained by removing all lines of $\mathcal{F}^{\prime}$ from $\mathcal{F}$

$\mathcal{P}^{\left[\mathcal{F}^{\prime}\right]}$ : coefficient associated to the skeleton $\mathcal{F}^{\prime} \quad \mathcal{K}^{\left[\mathcal{F} \backslash \mathcal{F}^{\prime}\right]}$ : coefficient associated to the graph $\mathcal{F} \backslash \mathcal{F}^{\prime}$

$\mathcal{N}(\mathcal{F})$ : the number of arrows (excluding the one which is attached to and points to the highest-weight node $h_{\rho(s)}$ in the gauge invariance induced identity eq. (3.1)) pointing away from the direction of roots.

$\mathcal{S}(\mathscr{U})$ : the number of spurious components in the final upper block

$\boldsymbol{\sigma}^{\mathcal{F}}$ : permutations allowed by graph $\mathcal{F}$

Type-I sector of a chain: A sector containing a type-1 line and possible type-2 lines whose arrows point to the two ends of the type-1 line

Type-II sector of a chain (the chain led by the highest weight node $h_{\rho(s)}$ ): A sector only containing type- 2 lines whose arrows point to the direction of the starting node $h_{\rho(s)}$ of the chain.

\footnotetext{
${ }^{10}$ As pointed in section 8 , the definition of skeleton for the identity eq. (8.11) is adjusted.
} 
Type-III sector of a chain: A sector only containing type-2 lines whose arrows point to the direction of the ending node of the chain

Type-I component $\mathscr{C}^{\mathrm{I}}$ : component containing a type- 1 line and possible type-2 lines whose arrows pointing to the direction of the two ends of the type- 1 line.

Kernel of a type-I component: the type-1 line in the type-I component

The top and bottom sides $\mathscr{C}_{t}^{\mathrm{I}}$ and $\mathscr{C}_{b}^{\mathrm{I}}$ of a type-I component $\mathscr{C}^{\mathrm{I}}$ : the two sides connected by the type- 1 line. The side containing the highest-weight node in $\mathscr{C}^{\mathrm{I}}$ is the top side $\mathscr{C}_{t}^{\mathrm{I}}$. The side which does not contain the highest-weight node in $\mathscr{C}^{\mathrm{I}}$ is the bottom side $\mathscr{C}_{b}^{\mathrm{I}}$.

Type-II component $\mathscr{C}^{\mathrm{II}}$ : the component containing the highest-weight graviton $h_{\rho(s)}$.

Type-III component $\mathscr{C}^{\mathrm{III}}: 11$ the component containing roots.

$\mathrm{R}_{\mathscr{C}}$ : the reference order of components The weight of a component: the position of a component in $\mathrm{R}_{\mathscr{C}}$

The upper and lower blocks $\mathscr{U}$ and $\mathscr{L}$ : the maximally connected graphs involving the type-II and the type-III components $\mathscr{C}^{\mathrm{II}}$ and $\mathscr{C}^{\mathrm{III}}$ respectively.

$\mathcal{T}$ : a tree graph with dashed lines (lines only imply the relative positions of nodes)

$\left.\mathcal{T}\right|_{a}:$ permutations established by $\mathcal{T}$ when considering $a$ as the leftmost node

$\oplus$ : union of two disjoint graphs

$\mathcal{T}-\mathcal{T}^{\prime}$ for a graph $\mathcal{T}$ its subgraph $\mathcal{T}^{\prime}$ : the graph obtained by removing all nodes in $\mathcal{T}^{\prime}$ and the lines attached to these nodes from $\mathcal{T}$

\section{B More examples for section 4}

In this part, we display more examples for section 4 .

\section{B.1 The identity with $\mathrm{H}=\left\{h_{1}\right\}$}

When the set $\mathrm{H}$ contains only one element $h_{1}$, there is only one possible structure of skeleton $\mathcal{F}^{\prime}$ (see figure $23(\mathrm{a})$ ). Physical graphs $\mathcal{F}$ containing this skeleton is given by figure 23 (b).

\footnotetext{
${ }^{11}$ In the example of figure 11 we use $\mathscr{A}$ and $\mathscr{B}$ to denote two Type-I components, use $\mathscr{C}$ to denote Type-II component and use $\mathscr{R}$ denote Type-III component.
} 


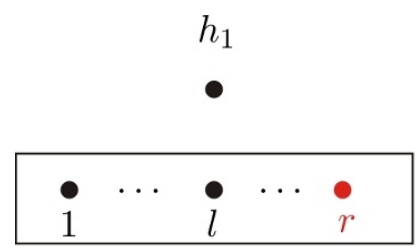

(a)

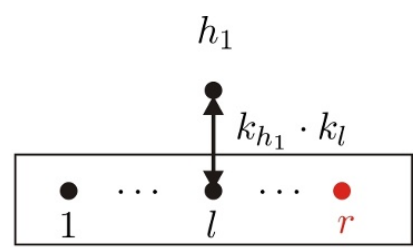

(b)

Figure 23. The graph (a) is the skeleton $\mathcal{F}^{\prime}$ for the gauge invariance induced identity (3.1) with $\mathrm{H}=\left\{h_{1}\right\}$. The graph (b) for $l \in\{1, \ldots, r-1\}$ is a typical graph $\mathcal{F}$ containing the skeleton (a).

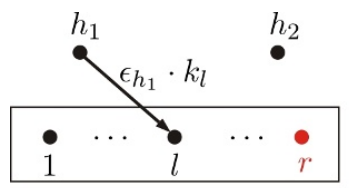

(a)

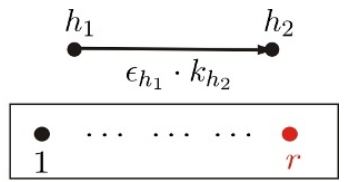

(b)

Figure 24. Graphs (a) and (b) are the skeletons $\mathcal{F}^{\prime}$ for the gauge invariance induced identity (3.1) with $\mathrm{H}=\left\{h_{1}, h_{2}\right\}$.

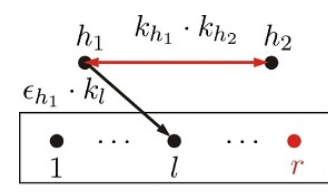

(a)

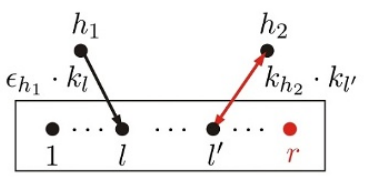

(b)

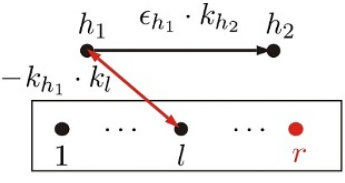

(c)

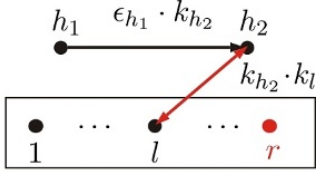

(d)

Figure 25. Graphs (a) and (b) are those graphs $\mathcal{F}$ containing the skeleton $\mathcal{F}^{\prime}=$ figure 24 (a). Graphs containing the skeleton $\mathcal{F}^{\prime}=$ figure $24(\mathrm{~b})$ are given by (c) and (d).

The 1.h.s. of the identity eq. (2.9) (equivalently eq. (3.5)) then reads

$$
T=1 \cdot\left[\sum_{l \in\{1,2, \ldots, r-1\}}\left(k_{h_{1}} \cdot k_{l}\right) \sum_{\boldsymbol{\sigma}^{l}} A\left(1, \boldsymbol{\sigma}^{l} \in\left\{2, \ldots, l,\left\{h_{1}\right\} \amalg\{l+1, \ldots, r-1\}\right\}, r\right)\right]
$$

The coefficient for a given permutation $\boldsymbol{\sigma} \in\left\{h_{1}\right\} \amalg\{2, \ldots, r-1\}$ are collected as $k_{h_{1}} \cdot Y_{h_{1}}(\boldsymbol{\sigma})$ thus eq. (B.1) can be written as $\mathcal{B}\left(1\left|\left\{h_{1}\right\},\{2, \ldots, r-1\}\right| r\right)$.

\section{B.2 The identity with $\mathrm{H}=\left\{h_{1}, h_{2}\right\}$}

In the identity (2.9) (or equivalently eq. (3.1)) with $\mathrm{H}=\left\{h_{1}, h_{2}\right\}$ and $\mathrm{R}=\left\{h_{1}, h_{2}\right\}$, two kinds of skeletons figure 24 (a), (b) have to be considered.

The skeleton figure 24 (a) with a given $l \in\{1,2, \ldots, r-1\}$ contributes a factor $\mathcal{P}^{\left[\mathcal{F}^{\prime}\right]}=$ $\epsilon_{h_{1}} \cdot k_{l}$. Graphs $\mathcal{F}$ containing the skeleton figure 24 (a) are displayed by figure 25 (a) and (b). The factor $\mathcal{K}^{\left[\mathcal{F} \backslash \mathcal{F}^{\prime}\right]}$ for $\mathcal{F}=$ figure 25 (a) is $k_{h_{2}} \cdot k_{h_{1}}$ and the corresponding permutations $\boldsymbol{\sigma}^{\mathcal{F}}$ are

$$
\boldsymbol{\sigma}^{\mathcal{F}} \in\left\{2, \ldots, l,\left\{h_{1}, h_{2}\right\} \amalg\{l+1, \ldots, r-1\}\right\}
$$


The factor $\mathcal{K}^{\left[\mathcal{F} \backslash \mathcal{F}^{\prime}\right]}$ for $\mathcal{F}=$ figure 25 (b) is $k_{h_{2}} \cdot k_{l^{\prime}}\left(l^{\prime}=\{1,2, \ldots, r-1\}\right)$ and the corresponding permutations $\boldsymbol{\sigma}^{\mathcal{F}}$ satisfy

$$
\begin{aligned}
& \boldsymbol{\sigma}^{\mathcal{F}} \in\left\{2, \ldots, l,\left\{h_{1}\right\} \uplus\left\{l+1, \ldots, l^{\prime},\left\{h_{2}\right\} \uplus\left\{l^{\prime}+1, \ldots, r-1\right\}\right\}\right\} \quad\left(1 \leq l \leq l^{\prime} \leq r-1\right) \\
& \boldsymbol{\sigma}^{\mathcal{F}} \in\left\{2, \ldots, l^{\prime},\left\{h_{2}\right\} \amalg\left\{l^{\prime}+1, \ldots, l,\left\{h_{1}\right\} \amalg\{l+1, \ldots, r-1\}\right\}\right\} \quad\left(1 \leq l^{\prime}<l \leq r-1\right) .
\end{aligned}
$$

Then the expression in the square brackets of eq. (3.4), which is associated to the skeleton $\mathcal{F}^{\prime}=$ figure 24 (a) for a given $l \in\{1,2, \ldots, r-1\}$, is explicitly written as

$$
\begin{aligned}
& \left(k_{h_{2}} \cdot k_{h_{1}}\right) \sum_{\boldsymbol{\sigma}} A\left(1, \boldsymbol{\sigma} \in\left\{2, \ldots, l,\left\{h_{1}, h_{2}\right\} ш\{l+1, \ldots, r-1\}\right\}, r\right) \\
& +\sum_{\substack{l^{\prime} \in\{1,2, \ldots, r-1\} \\
\text { s.t. } l^{\prime} \geq l}}\left(k_{h_{2}} \cdot k_{l^{\prime}}\right) \sum_{\boldsymbol{\sigma}} A\left(1, \boldsymbol{\sigma} \in\left\{2, \ldots, l,\left\{h_{1}\right\} ш\left\{l+1, \ldots, l^{\prime},\left\{h_{2}\right\} \amalg\left\{l^{\prime}+1, \ldots, r-1\right\}\right\}\right\}, r\right) \\
& +\sum_{\substack{l^{\prime} \in\{1,2, \ldots, r-1\} \\
\text { s.t. } l^{\prime}<l}}\left(k_{h_{2}} \cdot k_{l^{\prime}}\right) \sum_{\boldsymbol{\sigma}} A\left(1, \boldsymbol{\sigma} \in\left\{2, \ldots, l^{\prime},\left\{h_{2}\right\} \amalg\left\{l^{\prime}+1, \ldots, l,\left\{h_{1}\right\} \amalg\{l+1, \ldots, r-1\}\right\}\right\}, r\right) .
\end{aligned}
$$

The sum over all permutations $\sigma$ in the above expression can be obtained by summing over all possible $\boldsymbol{\sigma} \in\left\{h_{2}\right\} ш \boldsymbol{\beta}$ for a fixed $\boldsymbol{\beta} \in\left\{2, \ldots, l,\left\{h_{1}\right\} ш\{l+1, \ldots, r-1\}\right\}$ and then summing over all possible $\beta \in\left\{2, \ldots, l,\left\{h_{1}\right\} ш\{l+1, \ldots, r-1\}\right\}$. For a fixed $\beta \in\left\{2, \ldots, l,\left\{h_{1}\right\} ш\{l+1, \ldots, r-1\}\right\}$, the coefficients for $\boldsymbol{\sigma} \in\left\{h_{2}\right\} \amalg \boldsymbol{\beta}$ are collected as

$$
\begin{array}{ll}
\sum_{\substack{l^{\prime} \in\{1, \ldots, r-1\} \\
\sigma^{-1}\left(l^{\prime}\right)<\sigma^{-1}\left(h_{2}\right) \\
l^{\prime} \in\{1, \ldots, r-1\} \\
\sigma^{-1}\left(l^{\prime}\right)<\sigma^{-1}(l)}} k_{h_{2}} \cdot k_{l^{\prime}} \cdot k_{l^{\prime}}+\sum_{\substack{l^{\prime} \in\{1, \ldots, r-1\} \\
l^{\prime} \in\{1, \ldots, r-1\} \\
\sigma^{-1}\left(l^{\prime}\right)<\sigma^{-1}(l)}} k_{h_{2}} \cdot k_{h_{2}} \cdot k_{l^{\prime}} \sum_{\substack{\sigma^{\prime}(l)<\sigma^{-1}\left(l^{\prime}\right)<\sigma^{-1}\left(h_{2}\right) \\
l^{\prime} \in\{1, \ldots, r-1\} \\
\sigma^{-1}(l)<\sigma^{-1}\left(l^{\prime}\right)<\sigma^{-1}\left(h_{2}\right)}} k_{h_{2}} \cdot k_{l^{\prime}}+k_{h_{2}} \cdot k_{h_{1}} & \text { if } \sigma^{-1}\left(h_{2}\right)<\sigma^{-1}(l), \quad \text { if } \sigma^{-1}\left(h_{2}\right)>\sigma^{-1}\left(h_{1}\right),
\end{array}
$$

where the first line in the above expression comes from the last term of eq. (B.4), the second line gets contributions from both the second and the last terms of eq. (B.4), the third line gets contributions of all the three terms of eq. (B.4). Apparently, all the three cases can be uniformly written as $k_{h_{2}} \cdot X_{h_{2}}(\boldsymbol{\sigma})$ where $X_{h_{2}}(\boldsymbol{\sigma})$ is the sum of all elements (including the element 1) appearing on the l.h.s. of $h_{2}$ in the permutation $\sigma$. Therefore eq. (B.4) can be reorganized as

$\sum_{\sigma \in\left\{h_{2}\right\} ш \boldsymbol{\beta}}\left(k_{h_{2}} \cdot X_{h_{2}}(\boldsymbol{\sigma})\right) A(1, \boldsymbol{\sigma}, r)=\mathcal{B}\left(1\left|\left\{h_{2}\right\}, \boldsymbol{\beta}\right| r\right),\left(\right.$ for $\left.\boldsymbol{\beta} \in\left\{2, \ldots, l,\left\{h_{1}\right\} \amalg\{l+1, \ldots, r-1\}\right\}\right)$

which is a combination of the l.h.s. of fundamental BCJ relations.

The skeleton $\mathcal{F}^{\prime}=$ figure 24 (b) contributes a factor $P^{\left[\mathcal{F}^{\prime}\right]}=\epsilon_{h_{1}} \cdot k_{h_{2}}$. Graphs $\mathcal{F} \supset$ $\mathcal{F}^{\prime}$ containing the skeleton figure 24 (b) are presented by figure 25 (c) and (d) which 
are associated with the factors $\mathcal{K}^{\left[\mathcal{F} \backslash \mathcal{F}^{\prime}\right]}=-k_{h_{1}} \cdot k_{l}$ and $\mathcal{K}^{\left[\mathcal{F} \backslash \mathcal{F}^{\prime}\right]}=k_{h_{2}} \cdot k_{l}$, respectively. Permutations $\boldsymbol{\sigma}^{\mathcal{F}}$ corresponding to figure 25 (c) and (d) are

$$
\boldsymbol{\sigma}^{\mathcal{F}} \in\left\{2, \ldots, l,\left\{h_{1}, h_{2}\right\} \amalg\{l+1, \ldots, r-1\}\right\} \text { and } \boldsymbol{\sigma}^{\mathcal{F}} \in\left\{2, \ldots, l,\left\{h_{2}, h_{1}\right\} \amalg\{l+1, \ldots, r-1\}\right\},
$$

where $1 \leq l \leq r-1$. Thus the expression in the square brackets of eq. (3.4) for the skeleton figure 24 (b) is given by

$$
\begin{aligned}
& -\sum_{l \in\{1,2, \ldots, r-1\}}\left(k_{h_{1}} \cdot k_{l}\right) \sum_{\boldsymbol{\sigma}} A\left(1, \ldots, l, \boldsymbol{\sigma} \in\left\{h_{1}, h_{2}\right\}\right. \\
& \left.+\sum_{l \in\{1,2, \ldots, r-1\}}\left(k_{h_{2}} \cdot k_{l}\right) \sum_{\boldsymbol{\sigma}} A(1, \ldots, 1, \ldots, r-1\}, r\right)
\end{aligned}
$$

All possible permutations $\sigma$ in the above expression belong to $\{2, \ldots, r-1\} \amalg\left\{h_{1}\right\} \amalg\left\{h_{2}\right\}$, thus one can pick out $\sigma$ and collect all coefficients accompanying with it. The coefficient is $\left(k_{h_{1}} \cdot Y_{h_{1}}(\boldsymbol{\sigma})\right)$ for any $\boldsymbol{\sigma} \in\left\{h_{1}, h_{2}\right\} ш\{2, \ldots, r-1\}$ and $\left(k_{h_{2}} \cdot Y_{h_{2}}(\boldsymbol{\sigma})\right)$ for any $\boldsymbol{\sigma} \in$ $\left\{h_{2}, h_{1}\right\} \uplus\{2, \ldots, r-1\}$. Hence figure 24 (b) becomes

$$
-\sum_{\boldsymbol{\sigma} \in\left\{h_{1}, h_{2}\right\} \uplus\{2, \ldots, r-1\}}\left(k_{h_{1}} \cdot Y_{h_{1}}(\boldsymbol{\sigma})\right) A(1, \boldsymbol{\sigma}, r)+\sum_{\boldsymbol{\sigma} \in\left\{h_{2}, h_{1}\right\} \uplus\{2, \ldots, r-1\}}\left(k_{h_{2}} \cdot Y_{h_{2}}(\boldsymbol{\sigma})\right) A(1, \boldsymbol{\sigma}, r),
$$

which can be further arranged as

$$
\begin{aligned}
& -\sum_{\boldsymbol{\beta} \in\left\{h_{2}\right\} \uplus\{2, \ldots, r-1\}} \sum_{\boldsymbol{\sigma} \in\left\{h_{1}\right\} \uplus \boldsymbol{\beta}}\left(k_{h_{1}} \cdot X_{h_{1}}(\boldsymbol{\sigma})\right) A(1, \boldsymbol{\sigma}, r) \\
& +\sum_{\boldsymbol{\sigma} \in\left\{h_{2}, h_{1}\right\} \uplus\{2, \ldots, r-1\}}\left(k_{h_{2}} \cdot X_{h_{2}}(\boldsymbol{\sigma})+k_{h_{1}} \cdot X_{h_{1}}(\boldsymbol{\sigma})\right) A(1, \boldsymbol{\sigma}, r) \\
& =-\sum_{\boldsymbol{\beta} \in\left\{h_{2}\right\} \uplus\{2, \ldots, r-1\}} \mathcal{B}\left(1\left|\left\{h_{2}\right\}, \boldsymbol{\beta}\right| r\right)+\mathcal{B}\left(1\left|\left\{h_{2}, h_{1}\right\},\{2, \ldots, r-1\}\right| r\right) .
\end{aligned}
$$

Finally, the expression in the square brackets of eq. (3.4) for the skeleton figure 24 (b) has been expanded into the l.h.s. of BCJ relations.

\section{B.3 The identity with $\mathrm{H}=\left\{h_{1}, h_{2}, h_{3}\right\}$}

There are nine skeletons for the identity (2.9) (or equivalently eq. (3.1)) with three elements in $\mathbf{H}$ (The reference order is chosen as $\mathbf{R}=\left\{h_{1}, h_{2}, h_{3}\right\}$ ). In the following, we consider the skeletons figure 6 (a), (c) and (e) as examples and show that all contributions corresponding to each skeleton can be arranged as a proper combination of the l.h.s. of BCJ relations.

(1) The skeleton $\mathcal{F}^{\prime}=$ figure $6(\mathrm{a})$, which is associated with a factor $\mathcal{P}^{\left[\mathcal{F}^{\prime}\right]}=\left(\epsilon_{h_{1}} \cdot l\right)\left(\epsilon_{h_{2}} \cdot l^{\prime}\right)$ (for given $l, l^{\prime} \in\{1,2, \ldots, r-1\}$ ), consists of two disjoint components: the single node $h_{3}$ and a subgraph containing the elements $\{1, \ldots, r\} \cup\left\{h_{1}, h_{2}\right\}$. The possible relative orders $\beta$ for elements in $\{1, \ldots, r\} \cup\left\{h_{1}, h_{2}\right\}$ are

$$
\begin{aligned}
& \beta \in\left\{2, \ldots, l,\left\{h_{1}\right\} \uplus\left\{l+1, \ldots, l^{\prime},\left\{h_{2}\right\} \uplus\left\{l^{\prime}+1, \ldots, r-1\right\}\right\} \quad\left(1 \leq l \leq l^{\prime} \leq r-1\right)\right. \\
& \beta \in\left\{2, \ldots, l^{\prime},\left\{h_{2}\right\} \uplus\left\{l^{\prime}+1, \ldots, l,\left\{h_{1}\right\} \uplus\{l+1, \ldots, r-1\}\right\} \quad\left(1 \leq l^{\prime}<l \leq r-1\right) .\right.
\end{aligned}
$$




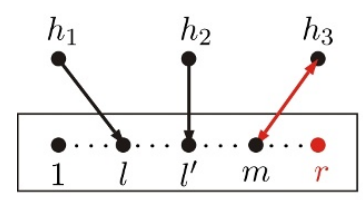

(a)

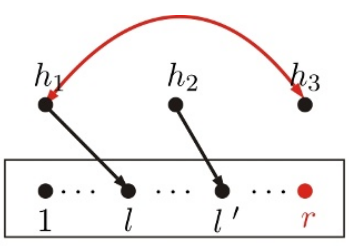

(b)

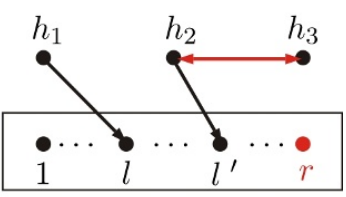

(c)

Figure 26. All graphs containing the skeleton $\mathcal{F}^{\prime}=$ figure 6 (a).

\begin{tabular}{|c|c|c|}
\hline Relative orders of $h_{1}, h_{2}$ and $h_{3}$ & Graphs $\mathcal{F} \supset \mathcal{F}^{\prime}$ & Sum of coefficients $(-1)^{\mathcal{N}(\mathcal{F})} \mathcal{K}^{\left[\mathcal{F} \backslash \mathcal{F}^{\prime}\right]}$ \\
\hline$\left\{h_{1}, h_{2}, h_{3}\right\}$ & Fig. 26 (a),(b),(c) & $k_{h_{3}} \cdot Y_{h_{3}}(\boldsymbol{\sigma})+k_{h_{3}} \cdot k_{h_{1}}+k_{h_{3}} \cdot k_{h_{2}}$ \\
$\left\{h_{1}, h_{3}, h_{2}\right\}$ & Fig. 26 (a),(b) & $k_{h_{3}} \cdot Y_{h_{3}}(\boldsymbol{\sigma})+k_{h_{3}} \cdot k_{h_{1}}$ \\
$\left\{h_{3}, h_{1}, h_{2}\right\}$ & Fig. 26 (a) & $k_{h_{3}} \cdot Y_{h_{3}}(\boldsymbol{\sigma})$ \\
$\left\{h_{2}, h_{1}, h_{3}\right\}$ & Fig. 26 (a),(b),(c) & $k_{h_{3}} \cdot Y_{h_{3}}(\boldsymbol{\sigma})+k_{h_{3}} \cdot k_{h_{1}}+k_{h_{3}} \cdot k_{h_{2}}$ \\
$\left\{h_{2}, h_{3}, h_{1}\right\}$ & Fig. 26 (a),(c) & $k_{h_{3}} \cdot Y_{h_{3}}(\boldsymbol{\sigma})+k_{h_{3}} \cdot k_{h_{2}}$ \\
$\left\{h_{3}, h_{2}, h_{1}\right\}$ & Fig. 26 (a) & $k_{h_{3}} \cdot Y_{h_{3}}(\boldsymbol{\sigma})$ \\
\hline
\end{tabular}

Table 2. Consider the graphs in figure 26, which contain the skeleton $\mathcal{F}^{\prime}=$ figure 6 (a). According to the refined graphic rule, the factor $(-1)^{\mathcal{N}(\mathcal{F})}$ for all the three graphs in figure 26 is $(-1)^{0}=1$.

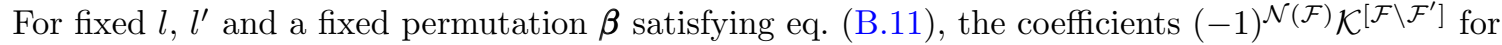
each permutation $\sigma \in \beta \amalg\left\{h_{3}\right\}$ are collected. For example, if the relative order of elements of $\left\{h_{1}, h_{2}, h_{3}\right\}$ in $\sigma \in \beta \amalg\left\{h_{3}\right\}$ is $h_{1}, h_{2}, h_{3}$, all graphs in figure 26 contribute to this permutation. The sum of figure 26 (a) for all $m$ satisfying $\boldsymbol{\sigma}^{-1}(m)<\boldsymbol{\sigma}^{-1}\left(h_{3}\right)$ provides $k_{h_{3}} \cdot Y_{h_{3}}(\boldsymbol{\sigma})$, while graphs figure 26 (b) and (c) contribute $k_{h_{3}} \cdot k_{h_{1}}$ and $k_{h_{3}} \cdot k_{h_{2}}$, respectively. Thus the total factor is $k_{h_{3}} \cdot Y_{h_{3}}(\boldsymbol{\sigma})+k_{h_{3}} \cdot k_{h_{1}}+k_{h_{3}} \cdot k_{h_{2}}$, as shown by the first row.

All graphs $\mathcal{F}$ containing the skeleton $\mathcal{F}^{\prime}=$ figure 6 (a) are provided by figure 26 (a), (b) and (c). For any fixed $l, l^{\prime}$ and a corresponding $\beta$, all possible permutations $\sigma$ corresponding to graphs figure 26 (a), (b) and (c) belong to $\beta ш\left\{h_{3}\right\}$. We can collect coefficients $(-1)^{\mathcal{N}(\mathcal{F})} \mathcal{K}^{\left[\mathcal{F} \backslash \mathcal{F}^{\prime}\right]}$ for each permutation $\sigma \in \beta ш\left\{h_{3}\right\}$. As shown by table 2 , coefficients for any permutation $\boldsymbol{\sigma} \in \boldsymbol{\beta} \amalg\left\{h_{3}\right\}$ can be uniformly written as $k_{h_{3}} \cdot X_{h_{3}}(\boldsymbol{\sigma})$. Thus the expression in the square brackets of eq. (3.5) for the skeleton $\mathcal{F}^{\prime}=$ figure 6 (a) (with given $l$ and $l^{\prime}$ ) is expressed by

$$
\sum_{\boldsymbol{\beta}}\left[\sum_{\boldsymbol{\sigma} \in\left\{h_{3}\right\} \uplus \boldsymbol{\beta}}\left(k_{h_{3}} \cdot X_{h_{3}}(\boldsymbol{\sigma})\right) A(1, \boldsymbol{\sigma}, r)\right]=\sum_{\boldsymbol{\beta}} \mathcal{B}\left(1\left|\left\{h_{3}\right\}, \boldsymbol{\beta}\right| r\right),
$$

where we summed over all $\beta$ satisfy eq. (B.11). Apparently, the expression in the square brackets in the above equation is the l.h.s. of a fundamental BCJ relation.

(2) The skeleton figure 6 (c) is associated with a factor $\mathcal{P}^{\mathcal{F}^{\prime}}=\left(\epsilon_{h_{1}} \cdot k_{h_{3}}\right)\left(\epsilon_{h_{2}} \cdot k_{l}\right)$ and consists of two disjoint components. Relative permutations for the elements in the component containing $\{1, \ldots, r\}$ are

$$
\beta \in\left\{2, \ldots, l,\left\{h_{2}\right\} ш\{l+1, \ldots, r-1\}\right\} \quad(1 \leq l \leq r-1) .
$$




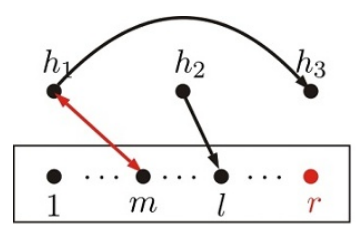

(a)

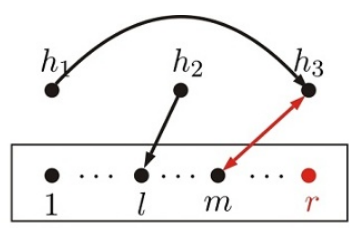

(b)

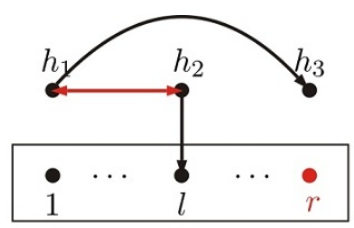

(c)

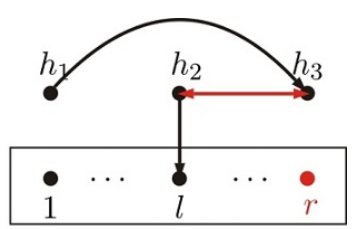

(d)

Figure 27. All graphs $\mathcal{F}$ containing the skeleton $\mathcal{F}^{\prime}=$ figure 6 (c).

\begin{tabular}{|c|c|c|}
\hline Relative orders of $h_{1}, h_{2}$ and $h_{3}$ & Graphs $\mathcal{F} \supset \mathcal{F}^{\prime}$ & Sum of coefficients $(-1)^{\mathcal{N}(\mathcal{F})} \mathcal{K}^{\left[\mathcal{F} \backslash \mathcal{F}^{\prime}\right]}$ \\
\hline$\left\{h_{1}, h_{2}, h_{3}\right\}$ & Fig. $27(\mathrm{a})$ & $-k_{h_{1}} \cdot Y_{h_{1}}(\boldsymbol{\sigma})$ \\
$\left\{h_{1}, h_{3}, h_{2}\right\}$ & Fig. 27 (a) & $-k_{h_{1}} \cdot Y_{h_{1}}(\boldsymbol{\sigma})$ \\
$\left\{h_{2}, h_{1}, h_{3}\right\}$ & Fig. 27 (a), (c) & $-\left(k_{h_{1}} \cdot Y_{h_{1}}(\boldsymbol{\sigma})+k_{h_{1}} \cdot k_{h_{2}}\right)$ \\
$\left\{h_{2}, h_{3}, h_{1}\right\}$ & Fig. 27 (b), (d) & $k_{h_{3}} \cdot Y_{h_{3}}(\boldsymbol{\sigma})+k_{h_{3}} \cdot k_{h_{2}}$ \\
$\left\{h_{3}, h_{1}, h_{2}\right\}$ & Fig. 27 (b) & $k_{h_{3}} \cdot Y_{h_{3}}(\boldsymbol{\sigma})$ \\
$\left\{h_{3}, h_{2}, h_{1}\right\}$ & Fig. 27 (b) & $k_{h_{3}} \cdot Y_{h_{3}}(\boldsymbol{\sigma})$ \\
\hline
\end{tabular}

Table 3. Consider the graphs in figure 27, which contain the skeleton $\mathcal{F}^{\prime}=$ figure 6 (c). According to the refined graphic rule, the factor $(-1)^{\mathcal{N}(\mathcal{F})}$ is -1 for figure 27 (a), (c), 1 for figure 27 (b), (d). For a given $l$ and a given $\beta$ satisfying eq. (B.13), permutations $\sigma$ corresponding to the skeleton $\mathcal{F}^{\prime}=$ figure 6 (c) belong to $\left\{h_{1}\right\} ш\left\{h_{3}\right\} \amalg \boldsymbol{\beta}$ for $\boldsymbol{\beta}$. For any $\boldsymbol{\sigma} \in\left\{h_{1}\right\} \amalg\left\{h_{3}\right\} \varpi \boldsymbol{\beta}$ we collect the coefficients $(-1)^{\mathcal{N}(\mathcal{F})} \mathcal{K}^{\left[\mathcal{F} \backslash \mathcal{F}^{\prime}\right]}$ together as we have done in the previous case. Each row shows the expression of the sum of $(-1)^{\mathcal{N}(\mathcal{F})} \mathcal{K}^{\left[\mathcal{F} \backslash \mathcal{F}^{\prime}\right]}$ for a given relative order of $h_{1}, h_{2}$ and $h_{3}$.

All graphs $\mathcal{F}$ satisfying $\mathcal{F} \supset \mathcal{F}^{\prime}=$ figure 6 (c) are presented by figure 27 and all possible permutations $\boldsymbol{\sigma}$ allowed by the graphs in figure 27 belong to $\beta \amalg\left\{h_{1}\right\} \amalg\left\{h_{3}\right\}$ (for a given $\beta$ in eq. (B.13)). To obtain the expression in the brackets in eq. (3.5) corresponding to the skeleton figure 6 (c) for a given $l \in\{1, \ldots, r-1\}$, we can collect the coefficients for any given permutation $\sigma \in \beta \amalg\left\{h_{1}\right\} ш\left\{h_{3}\right\}$ (see table 3). Then sum over all possible $\boldsymbol{\sigma} \in \boldsymbol{\beta} \amalg\left\{h_{1}\right\} \amalg\left\{h_{3}\right\}$ and all possible $\boldsymbol{\beta}$ satisfying eq. (B.13). From table 3, we find that the coefficients in the first three rows and the last three rows can be respectively written as $-k_{h_{1}} \cdot X_{h_{1}}(\boldsymbol{\sigma})$ and $k_{h_{3}} \cdot X_{h_{3}}(\boldsymbol{\sigma})$. Thus the expression in the square brackets of eq. (3.5) for the skeleton figure 6 (c) $(l \in\{1, \ldots, r-1\})$ is given by

$$
-\sum_{\boldsymbol{\beta}}\left[\sum_{\boldsymbol{\sigma} \in\left\{h_{1}, h_{3}\right\} \omega \boldsymbol{\beta}}\left(k_{h_{1}} \cdot X_{h_{1}}(\boldsymbol{\sigma})\right) A(1, \boldsymbol{\sigma}, r)\right]+\sum_{\boldsymbol{\beta}}\left[\sum_{\boldsymbol{\sigma} \in\left\{h_{3}, h_{1}\right\} \omega \boldsymbol{\beta}}\left(k_{h_{3}} \cdot X_{h_{3}}(\boldsymbol{\sigma})\right) A(1, \boldsymbol{\sigma}, r)\right] \text {, }
$$

in which the first summation is taken over all $\beta$ satisfying eq. (B.13). The first term in the above expression can be rewritten as

$$
\begin{aligned}
& -\sum_{\boldsymbol{\beta}}\left[\sum_{\boldsymbol{\sigma} \in\left\{h_{1}\right\} \uplus\left\{h_{3}\right\} \uplus \boldsymbol{\beta}}\left(k_{h_{1}} \cdot X_{h_{1}}(\boldsymbol{\sigma})\right) A(1, \boldsymbol{\sigma}, r)-\sum_{\boldsymbol{\sigma} \in\left\{h_{3}, h_{1}\right\} \omega \boldsymbol{\beta}}\left(k_{h_{1}} \cdot X_{h_{1}}(\boldsymbol{\sigma})\right) A(1, \boldsymbol{\sigma}, r)\right] \\
& =-\sum_{\boldsymbol{\beta}} \sum_{\boldsymbol{\rho}} \mathcal{B}\left(1\left|\left\{h_{1}\right\}, \boldsymbol{\rho} \in\left\{h_{3}\right\} \amalg \boldsymbol{\beta}\right| r\right)+\sum_{\boldsymbol{\beta}}\left[\sum_{\boldsymbol{\sigma} \in\left\{h_{3}, h_{1}\right\} \omega \boldsymbol{\beta}}\left(k_{h_{1}} \cdot X_{h_{1}}(\boldsymbol{\sigma})\right) A(1, \boldsymbol{\sigma}, r)\right] .
\end{aligned}
$$




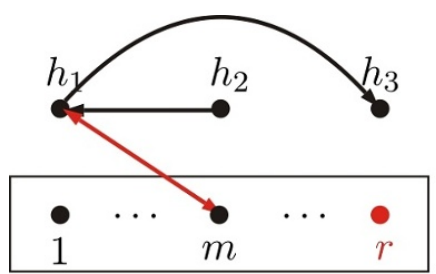

(a)

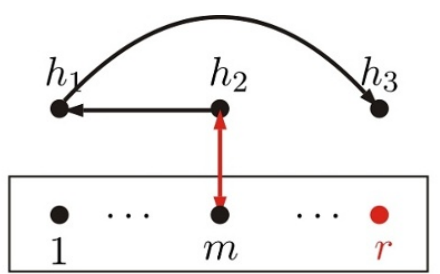

(b)

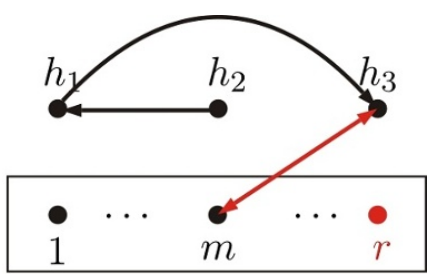

(c)

Figure 28. All graphs $\mathcal{F}$ containing the skeleton $\mathcal{F}^{\prime}=$ figure $6(\mathrm{e})$.

\begin{tabular}{|c|c|c|}
\hline Relative orders of $h_{1}, h_{2}$ and $h_{3}$ & Graphs $\mathcal{F} \supset \mathcal{F}^{\prime}$ & Coefficients $(-1)^{\mathcal{N}(\mathcal{F})} \mathcal{K}^{\left[\mathcal{F} \backslash \mathcal{F}^{\prime}\right]}$ \\
\hline$\left\{h_{1}, h_{2}, h_{3}\right\}$ & Fig. 28 (a) & $-k_{h_{1}} \cdot Y_{h_{1}}(\boldsymbol{\sigma})$ \\
$\left\{h_{1}, h_{3}, h_{2}\right\}$ & Fig. 28 (a) & $-k_{h_{1}} \cdot Y_{h_{1}}(\boldsymbol{\sigma})$ \\
$\left\{h_{2}, h_{1}, h_{3}\right\}$ & Fig. 28 (b) & $k_{h_{2}} \cdot Y_{h_{2}}(\boldsymbol{\sigma})$ \\
$\left\{h_{3}, h_{1}, h_{2}\right\}$ & Fig. 28 (c) & $k_{h_{3}} \cdot Y_{h_{3}}(\boldsymbol{\sigma})$ \\
\hline
\end{tabular}

Table 4. Total coefficients $(-1)^{\mathcal{N}(\mathcal{F})} \mathcal{K}^{\left[\mathcal{F} \backslash \mathcal{F}^{\prime}\right]}$ for permutations allowed by the graphs in figure 28 .

Since the sum of the last term on the second line of the above equation and the second term of eq. (B.14) is just a combination of l.h.s. of BCJ relations $\sum_{\boldsymbol{\beta}} \mathcal{B}\left(1\left|\left\{h_{3}, h_{1}\right\}, \boldsymbol{\beta}\right| r\right)$, eq. (B.14) is finally arranged as

$$
-\sum_{\boldsymbol{\beta}} \sum_{\boldsymbol{\rho}} \mathcal{B}\left(1\left|\left\{h_{1}\right\}, \boldsymbol{\rho} \in\left\{h_{3}\right\} ш \boldsymbol{\beta}\right| r\right)+\sum_{\boldsymbol{\beta}} \mathcal{B}\left(1\left|\left\{h_{3}, h_{1}\right\}, \boldsymbol{\beta}\right| r\right),
$$

the first summation in each term is taken over all $\beta$ satisfying eq. (B.13). Thus the expression in the brackets in eq. (3.5) for the skeleton figure 6 (c) is written as a combination of the 1.h.s. of BCJ relations.

(3) The skeleton $\mathcal{F}^{\prime}=$ figure 6 (e) gives a factor $\mathcal{P}^{\left[\mathcal{F}^{\prime}\right]}=\left(\epsilon_{h_{1}} \cdot k_{h_{3}}\right)\left(\epsilon_{h_{2}} \cdot k_{h_{1}}\right)$ and consists of two disjoint components. All possible graphs satisfying $\mathcal{F} \supset \mathcal{F}^{\prime}$ are displayed in figure 28. Relative orders of $h_{1}, h_{2}$ and $h_{3}$, allowed by the graphs in figure 28 are $\left\{h_{1},\left\{h_{2}\right\} \amalg\left\{h_{3}\right\}\right\}$, $\left\{h_{2}, h_{1}, h_{3}\right\}$ and $\left\{h_{3}, h_{1}, h_{2}\right\}$, whose coefficients $\mathcal{K}^{\left[\mathcal{F} \backslash \mathcal{F}^{\prime}\right]}$ are collected in table 4 . Thus the total contribution from the skeleton reads

$$
\begin{aligned}
& \sum_{\boldsymbol{\sigma} \in\{2, \ldots, r-1\} \uplus\left\{h_{1},\left\{h_{2}\right\} \uplus\left\{h_{3}\right\}\right\}}\left(-k_{h_{1}} \cdot Y_{h_{1}}(\boldsymbol{\sigma})\right) A(1, \boldsymbol{\sigma}, r) \\
& +\sum_{\boldsymbol{\sigma} \in\{2, \ldots, r-1\} \uplus\left\{h_{2}, h_{1}, h_{3}\right\}}\left(k_{h_{2}} \cdot Y_{h_{2}}(\boldsymbol{\sigma})\right) A(1, \boldsymbol{\sigma}, r)+\sum_{\boldsymbol{\sigma} \in\{2, \ldots, r-1\} \uplus\left\{h_{3}, h_{1}, h_{2}\right\}}\left(k_{h_{3}} \cdot Y_{h_{3}}(\boldsymbol{\sigma})\right) A(1, \boldsymbol{\sigma}, r) .
\end{aligned}
$$

When expressing the last two terms respectively by

$$
\sum_{\boldsymbol{\beta} \in\{2, \ldots, r-1\} \uplus\left\{h_{1}, h_{3}\right\}} \mathcal{B}\left(1\left|\left\{h_{2}\right\}, \boldsymbol{\beta}\right| r\right)-\left[\sum_{\boldsymbol{\sigma} \in\{2, \ldots, r-1\} \uplus\left\{h_{1},\left\{h_{2}\right\} \uplus\left\{h_{3}\right\}\right\}}\left(k_{h_{2}} \cdot X_{h_{2}}(\boldsymbol{\sigma})\right) A(1, \boldsymbol{\sigma}, r)\right]
$$


and

$$
\sum_{\boldsymbol{\beta} \in\{2, \ldots, r-1\} \uplus\left\{h_{1}, h_{2}\right\}} \mathcal{B}\left(1\left|\left\{h_{3}\right\}, \boldsymbol{\beta}\right| r\right)-\left[\sum_{\boldsymbol{\sigma} \in\{2, \ldots, r-1\} \uplus\left\{h_{1},\left\{h_{2}\right\} \uplus\left\{h_{3}\right\}\right\}}\left(k_{h_{3}} \cdot X_{h_{3}}(\boldsymbol{\sigma})\right) A(1, \boldsymbol{\sigma}, r)\right],
$$

eq. (B.17) becomes

$$
\begin{aligned}
& \sum_{\boldsymbol{\beta} \in\{2, \ldots, r-1\} \uplus\left\{h_{1}, h_{3}\right\}} \mathcal{B}\left(1\left|\left\{h_{2}\right\}, \boldsymbol{\beta}\right| r\right)+\sum_{\boldsymbol{\beta} \in\{2, \ldots, r-1\} \uplus\left\{h_{1}, h_{2}\right\}} \mathcal{B}\left(1\left|\left\{h_{3}\right\}, \boldsymbol{\beta}\right| r\right) \\
& -\sum_{\boldsymbol{\rho} \in\left\{h_{1},\left\{h_{2}\right\} \uplus\left\{h_{3}\right\}\right\}} \mathcal{B}(1|\boldsymbol{\rho},\{2, \ldots, r-1\}| r),
\end{aligned}
$$

which is a combination of the l.h.s. of BCJ relations.

\section{Graphs for the $\mathrm{H}=11$ example}

All physical graphs obtained by construction-2 are given by figure 29 (a1), (b1), (c1), (d1), figure 30 (a1), (b1), (c1), (d1) and figure 31 (a1), (b1), (c1), (d1). Each graph with a given dashed double arrow line gives a possible configuration. The corresponding spurious graphs are provided by figure 29 (a2), (b2), (c2), (d2), figure 30 (a2), (b2), (c2), (d2) and figure 31 (a2), (b2), (c2), (d2). Dashed doulbe arrow lines in spurious graphs are assciated with numbers and signs. One finds that the two spurious graphs labeled by the same number and distinct sign should cancel out. The two spurious graphs with the dashed lines labeled by the same number but opposite signs cancel with each other.

\section{General rules for the construction of physical graphs}

All (physical) graphs $\mathscr{F}$ s.t. $\mathscr{F} \supset \mathscr{F}^{\prime}$ in eq. (3.5) should be directly derived from the refined graphic rule. Now we show the general construction rule for all $\mathscr{F}$ (s.t. $\mathscr{F} \supset \mathscr{F}^{\prime}$ ), which is based the on refined graphic rule. We further prove that all the physical graphs obtained in section 6 (by connecting final upper and lower blocks) precisely match all the graphs produced by the rule introduced in this section. Further noting that all spurious graphs cancel out, the expression in the square brackets of eq. (3.5) is thus equivalent with eq. (6.5).

As pointed in section 5, each skeleton $\mathcal{F}^{\prime}$ in eq. (3.5) at least contains a type-II component $\mathscr{C}^{\mathrm{II}}$ and a type-III component $\mathscr{C}^{\mathrm{III}}$. Moreover, $N$ type-I components $\mathscr{C}_{1}^{\mathrm{I}}, \mathscr{C}_{2}^{\mathrm{I}}, \ldots$, $\mathscr{C}_{N}^{\mathrm{I}}$ may also be involved. To construct all physical graphs for a skeleton with $N+2$ components, we should connect these components together via $N+1$ type-3 lines in a proper way. By a direct analysis of the refined graphic rule in section 3, this can be achieved by the following rule. 


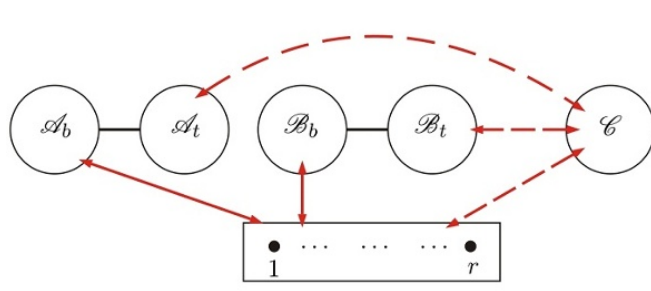

(a1)

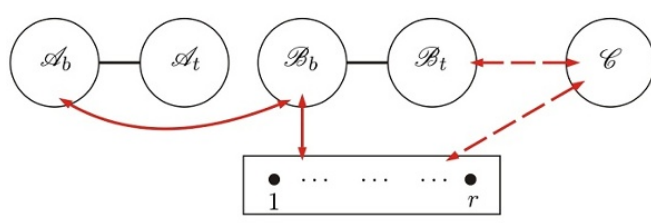

(b1)

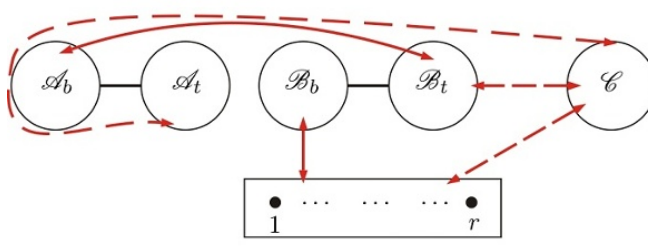

(c1)

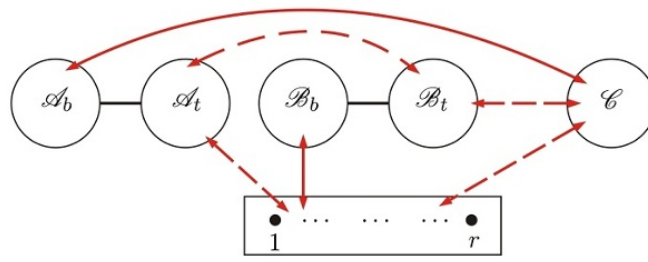

(d1)

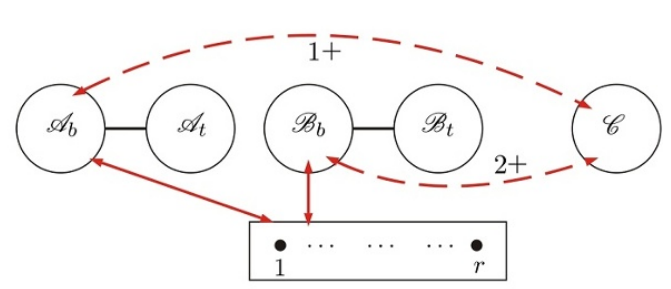

(a2)

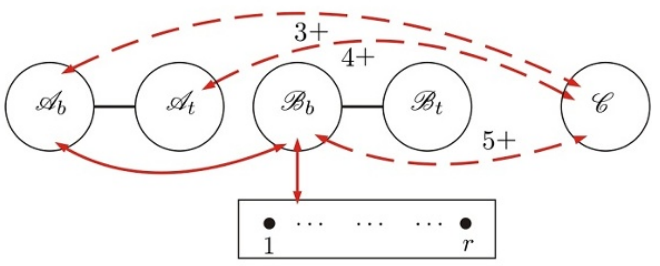

(b2)

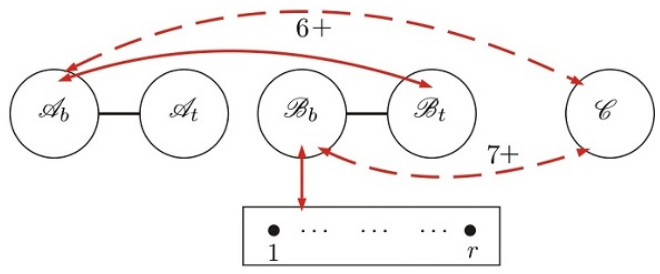

(c2)

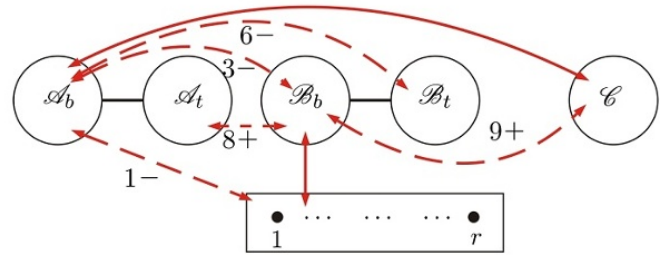

(d2)

Figure 29. Physical graphs (a1), (b1), (c1), (d1) for the skeleton figure 11 by construction-2 and the corresponding spurious graphs (a2), (b2), (c2), (d2): part 1.

\section{The construction rule}

Step-1. Assuming that the reference order of all type-I and type-II components is given by $\mathrm{R}_{\mathscr{C}}=\left\{\mathscr{C}_{1}^{\mathrm{I}}, \mathscr{C}_{2}^{\mathrm{I}}, \ldots, \mathscr{C}_{N}^{\mathrm{I}}, \mathscr{C}^{\mathrm{II}}\right\}$, we pick out the type-II component $\mathscr{C}^{\mathrm{II}}$ (the highest-weight component) as well as arbitrary type-I components $\mathscr{C}_{a_{1}}^{\mathrm{I}}, \mathscr{C}_{a_{2}}^{\mathrm{I}}, \ldots, \mathscr{C}_{a_{i}}^{\mathrm{I}}$ (not necessary to preserve the relative order in $\mathrm{R}_{\mathscr{C}}$ ) and construct a chain $\mathbb{C H}$ of components, whose starting component is $\mathscr{C}^{\mathrm{II}}$ and internal components are $\mathscr{C}_{a_{1}}^{\mathrm{I}}, \mathscr{C}_{a_{2}}^{\mathrm{I}}, \ldots, \mathscr{C}_{a_{i}}^{\mathrm{I}}$, towards $\mathscr{C}^{\mathrm{III}}$ :

$$
\mathbb{C H}=\left[\mathscr{C}^{\mathrm{II}},\left(\mathscr{C}_{a_{i}}^{\mathrm{I}}\right)_{t(\text { or } b)}-\left(\mathscr{C}_{a_{i}}^{\mathrm{I}}\right)_{b(\text { or } t)}, \ldots,\left(\mathscr{C}_{a_{1}}^{\mathrm{I}}\right)_{t(\text { or } b)}-\left(\mathscr{C}_{a_{1}}^{\mathrm{I}}\right)_{b(\text { or } t)}, \mathscr{C}^{\mathrm{III}}\right] .
$$

On this chain, two adjacent regions separated by commas $\left(\right.$ e.g. $\mathscr{C}^{\mathrm{II}}$ and $\left.\left(\mathscr{C}_{a_{i}}^{\mathrm{I}}\right)_{t(\text { or } b)}\right)$ are connected via a type-3 line. This chain is naturally physical because the structures figure 12 (a) and (b) are avoided. Redefine the reference order by $\mathrm{R}_{\mathscr{C}} \rightarrow \mathrm{R}_{\mathscr{C}}^{\prime} \equiv \mathrm{R}_{\mathscr{C}} \backslash$ $\left\{\mathscr{C}^{\mathrm{II}}, \mathscr{C}_{a_{1}}^{\mathrm{I}}, \mathscr{C}_{a_{2}}^{\mathrm{I}}, \ldots, \mathscr{C}_{a_{i}}^{\mathrm{I}}\right\}$ 


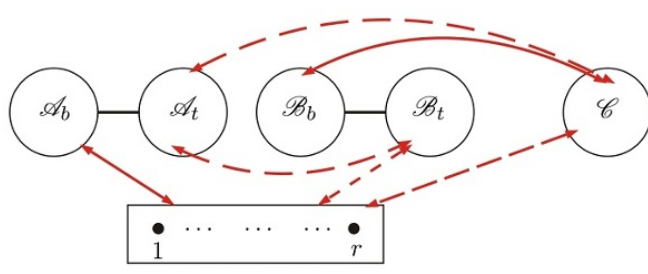

(a1)

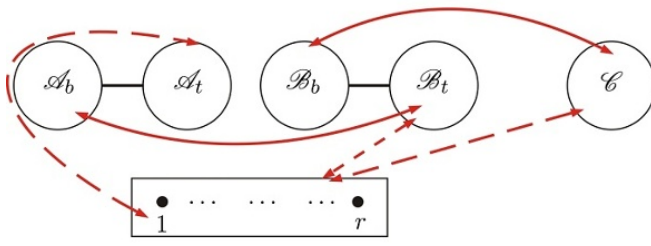

(b1)

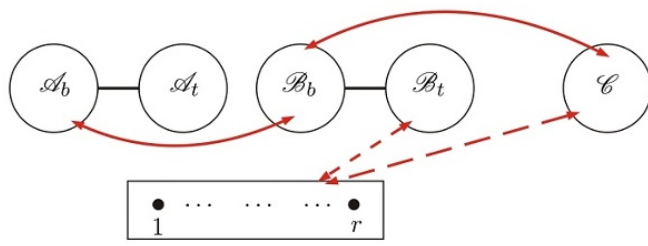

(c1)

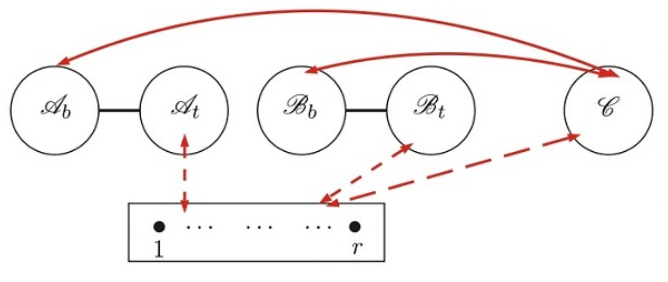

(d1)

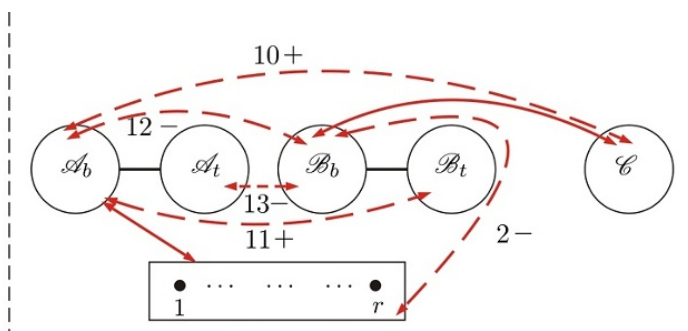

(a2)

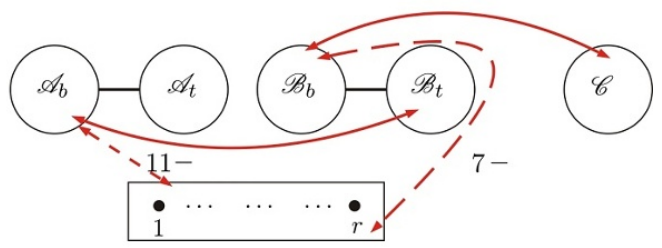

(b2)

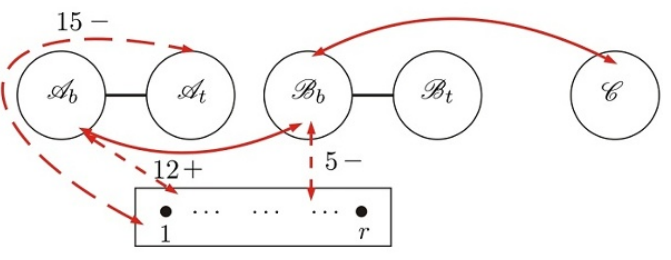

(c2)

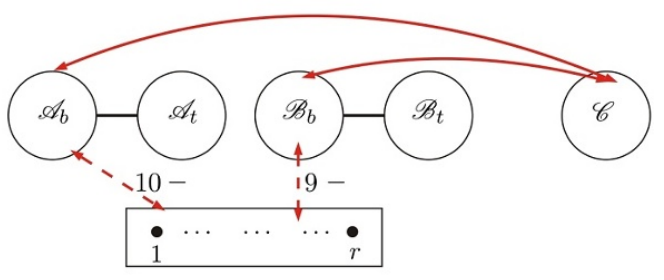

(d2)

Figure 30. Physical graphs (a1), (b1), (c1), (d1) for the skeleton figure 11 by construction-2 and the corresponding spurious graphs (a2), (b2), (c2), (d2): part 2.

Step-2. After the redefinition in the previous step, the ordered set $\mathrm{R}_{\mathscr{C}}=\mathrm{R}_{\mathscr{C}}^{\prime}$ only consists of type-I components. Now we pick out the highest-weight component, say $\mathscr{C}_{a_{i^{\prime}}^{\prime}}^{\mathrm{I}}$ as well as arbitrary type-I components $\mathscr{C}_{a_{1}^{\prime}}^{\mathrm{I}}, \ldots, \mathscr{C}_{a_{i^{\prime}}^{\prime}}^{\mathrm{I}}$ from $\mathrm{R}_{\mathscr{C}}^{\prime}$ and construct a chain $\mathbb{C H}^{\prime}$ towards a component $\mathscr{C} \in\left\{\mathscr{C}^{\mathrm{II}}, \mathscr{C}_{a_{1}}^{\mathrm{I}}, \mathscr{C}_{a_{2}}^{\mathrm{I}}, \ldots, \mathscr{C}_{a_{i}}^{\mathrm{I}}, \mathscr{C}^{\mathrm{III}}\right\}$, which has been used in the previous step, as follows:

$$
\mathbb{C} \mathbb{H}^{\prime}=\left[\left(\mathscr{C}_{a_{i^{\prime}}^{\prime}}^{\mathrm{I}}\right)_{t(\text { or } b)}-\left(\mathscr{C}_{a_{i^{\prime}}^{\prime}}^{\mathrm{I}}\right)_{b(\text { or } t)},\left(\mathscr{C}_{a_{i^{\prime}-1}^{\prime}}^{\mathrm{I}}\right)_{t(\text { or } b)}-\left(\mathscr{C}_{a_{i^{\prime}-1}^{\prime}}^{\mathrm{I}}\right)_{b(\text { or } t)}, \ldots,\left(\mathscr{C}_{a_{1}^{\prime}}^{\mathrm{I}}\right)_{t(\text { or } b)}-\left(\mathscr{C}_{a_{1}^{\prime}}^{\mathrm{I}}\right)_{b(\text { or } t)}, \mathscr{C}\right]
$$

Redefine the reference order by $\mathrm{R}_{\mathscr{C}} \rightarrow \mathrm{R}_{\mathscr{C}}^{\prime \prime} \equiv \mathrm{R}_{\mathscr{C}}^{\prime} \backslash\left\{\mathscr{C}_{a_{1}^{\prime}}^{\mathrm{I}}, \mathscr{C}_{a_{2}^{\prime}}^{\mathrm{I}}, \ldots, \mathscr{C}_{a_{i^{\prime}}^{\prime}}^{\mathrm{I}}\right\}$ 


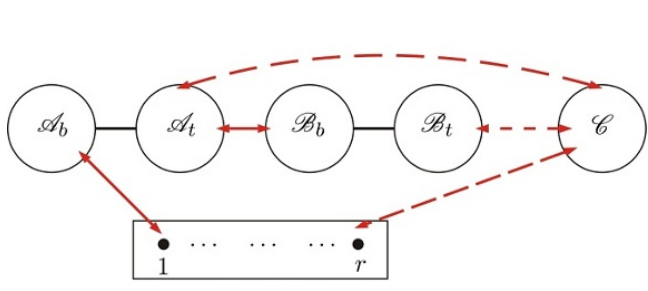

(a1)

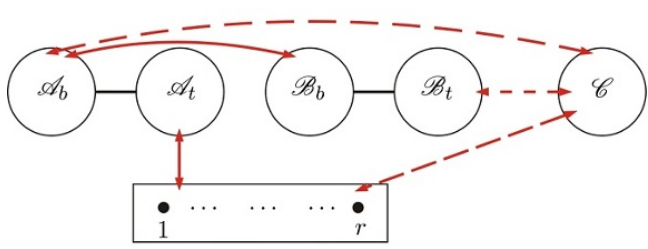

(b1)

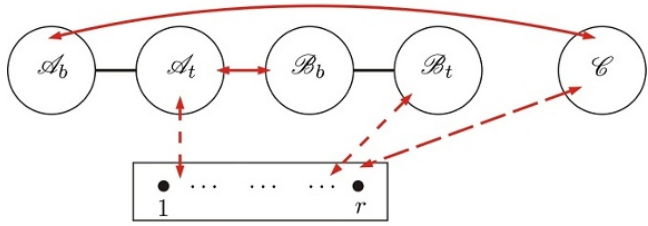

(c1)
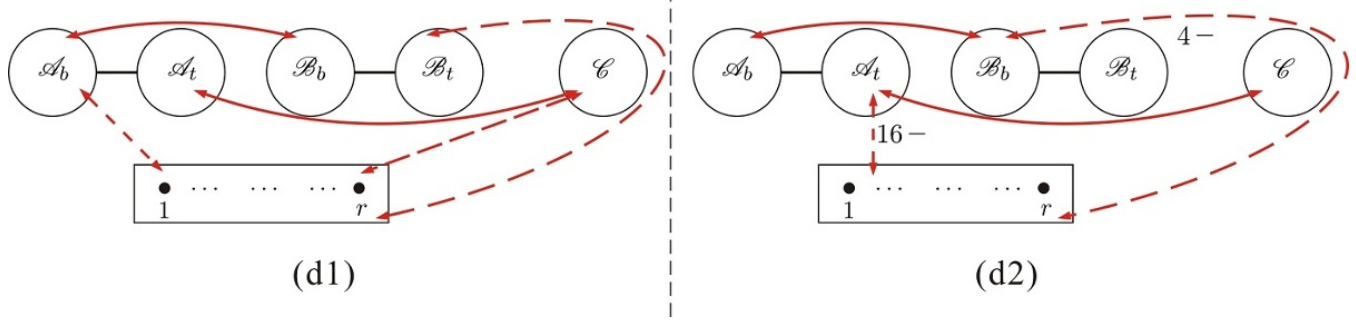

(d2)

Figure 31. Physical graphs (a1), (b1), (c1), (d1) for the skeleton figure 11 by construction- 2 and the corresponding spurious graphs (a2), (b2), (c2), (d2): part 3.

Step-3. Repeat the above steps. In each step, construct a chain, whose starting component is the highest-weight one in $\mathrm{R}_{\mathscr{C}}$ redefined by the previous step, towards an arbitrary component which has been used in the previous steps. Then redefine $\mathrm{R}_{\mathscr{C}}$ by removing the starting and internal components which have been used in this step. This procedure is terminated till the ordered set $\mathrm{R}_{\mathscr{C}}$ becomes empty. We obtain a physical graph containing the skeleton $\mathcal{F}^{\prime}$. All graphs (all possible chain structures of components and all possible choices of the end nodes of each type-3 lines) together form the set of all physical graphs $\mathcal{F}$ for the skeleton $\mathcal{F}^{\prime}$. By this rule, one can reproduce all the physical graphs figure 29 (a1), (b1), (c1), (d1), figure 30 (a1), (b1), (c1), (d1) and figure 31 (a1), (b1), (c1), (d1) corresponding to the skeleton figure 11 . 


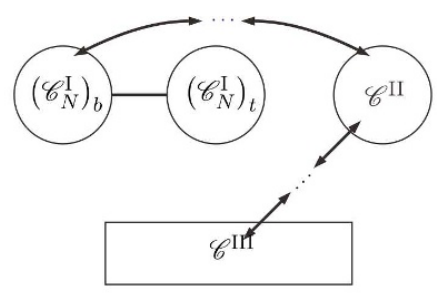

(a)

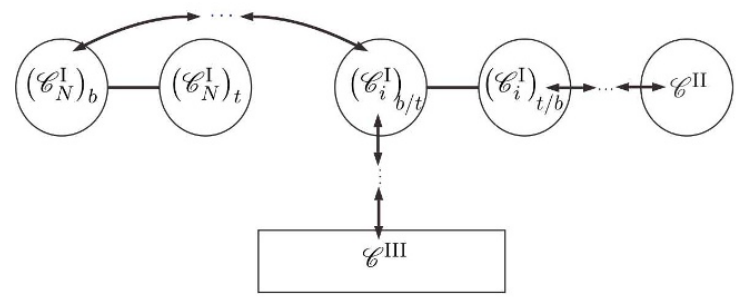

(c)

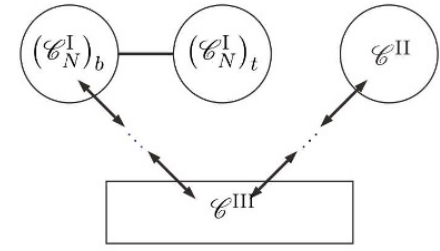

(b)

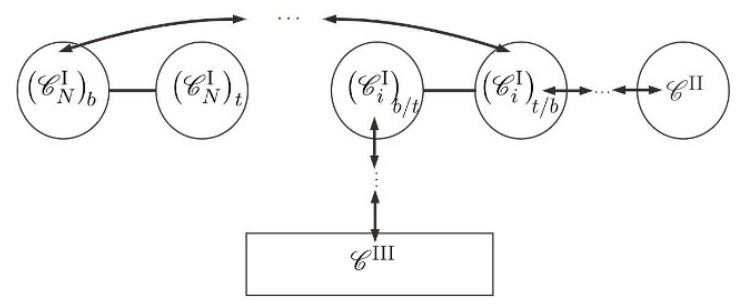

(d)

Figure 32. According to the construction rule, the chain starts at the component $\mathscr{C}_{N}^{\mathrm{I}}$ must ends at (i) the starting component (graph (a)), (ii) the ending component (graph (b)) or (iii) an internal component (graph (c) and (d)) of the chain led by $\mathscr{C}^{\mathrm{II}}$. According to section 6.1 , the chain led by $\mathscr{C}_{N}^{\mathrm{I}}$ in graphs (a)-(d) must be of the form (i) $\mathscr{U}^{\prime}=\left[\left(\mathscr{C}_{N}^{\mathrm{I}}\right)_{t}-\left(\mathscr{C}_{N}^{\mathrm{I}}\right)_{b}, \ldots, \mathscr{U}=\mathscr{C}^{\mathrm{II}}\right]$ (graph (a)), (ii) $\mathscr{L}^{\prime}=\left[\left(\mathscr{C}_{N}^{\mathrm{I}}\right)_{t}-\left(\mathscr{C}_{N}^{\mathrm{I}}\right)_{b}, \ldots, \mathscr{L}=\mathscr{C}_{\mathrm{III}}\right](\operatorname{graph}(\mathrm{b})),\left(\right.$ iii) $\mathscr{U}^{\prime}=\left[\left(\mathscr{C}_{N}^{\mathrm{I}}\right)_{t}-\left(\mathscr{C}_{N}^{\mathrm{I}}\right)_{b}, \ldots, \mathscr{U}=\mathscr{C}^{\mathrm{II}}\right]$ (graph (c)) and (iv) $\mathscr{L}^{\prime}=\left[\left(\mathscr{C}_{N}^{\mathrm{I}}\right)_{t}-\left(\mathscr{C}_{N}^{\mathrm{I}}\right)_{b}, \ldots,\left(\mathscr{C}_{i}^{\mathrm{I}}\right)_{t}-\left(\mathscr{C}_{i}^{\mathrm{I}}\right)_{b}, \ldots, \mathscr{L}=\mathscr{C}^{\mathrm{III}}\right]$ (graph (d)).

The above construction rule naturally inherits the chain structures of the refined graphic rule. More specifically, the chains of components are constructed by keeping track of the chains led by the highest-weight nodes in them. In the following, we prove that there exists a one-to-one correspondence between all graphs $\mathscr{F}\left(\mathscr{F} \supset \mathscr{F}^{\prime}\right)$ obtained by the above rule and all graphs constructed by connecting all possible configurations of the final upper and lower blocks corresponding to the same skeleton $\mathscr{F}^{\prime}$.

\section{The equivalence between the construction rule and the approach in section 6}

Any graph constructed by the rule given in the current section can be understood as follows. We define the upper block by $\mathscr{U}=\mathscr{C}^{\mathrm{II}}$, the lower block by $\mathscr{L}=\mathscr{C}^{\mathrm{III}}$ and the reference order $\mathrm{R}_{\mathscr{C}}^{\mathrm{I}} \equiv \mathrm{R}_{\mathscr{C}} \backslash \mathscr{C}^{\mathrm{II}}=\left\{\mathscr{C}_{1}^{\mathrm{I}}, \mathscr{C}_{2}^{\mathrm{I}}, \ldots, \mathscr{C}_{N}^{\mathrm{I}}\right\}$. For a given graph, we find out the chain led by the highest-weight type-I component $\mathscr{C}_{N}^{\mathrm{I}}$ in the reference order $\mathrm{R}_{\mathscr{C}}^{\mathrm{I}}$. According to the construction rule, this chain can be ended at any component on the chain led by the type-II component $\mathscr{C}^{\mathrm{II}}$ because the weight of $\mathscr{C}^{\mathrm{II}}$ is higher than $\mathscr{C}_{N}^{\mathrm{I}}$. Since the chain that is led by $\mathscr{C}^{\mathrm{II}}$ starts from $\mathscr{C}^{\mathrm{II}}$, ends at $\mathscr{C}^{\mathrm{III}}$ and can also have possible internal type-I components, the ending component of the chain led by $\mathscr{C}_{N}^{\mathrm{I}}$ could be (i) $\mathscr{U}=\mathscr{C}^{\mathrm{II}}$ (figure 32 (a)), (ii) $\mathscr{L}=\mathscr{C}^{\text {III }}$ (figure 32 (b)) or (iii) any internal type-I component of the chain led by $\mathscr{C}^{\mathrm{II}}$ (figure $32(\mathrm{c})$ and (d)). For the case (i), we redefine the upper block by the chain $\mathscr{U} \rightarrow \mathscr{U}^{\prime}=\left[\left(\mathscr{C}_{N}^{\mathrm{I}}\right)_{t}-\left(\mathscr{C}_{N}^{\mathrm{I}}\right)_{b}, \ldots, \mathscr{U}=\mathscr{C}^{\mathrm{II}}\right]$ that starts at $\mathscr{C}_{N}^{\mathrm{I}}$ and ends at the upper block $\mathscr{U}=\mathscr{C}^{\mathrm{II}}$. For the case (ii), we redefine the lower block by the chain $\mathscr{L} \rightarrow \mathscr{L}^{\prime}=$ $\left[\left(\mathscr{C}_{N}^{\mathrm{I}}\right)_{t}-\left(\mathscr{C}_{N}^{\mathrm{I}}\right)_{b}, \ldots, \mathscr{L}=\mathscr{C}^{\mathrm{III}}\right]$ that starts from $\mathscr{C}_{N}^{\mathrm{I}}$ and ends at the lower block $\mathscr{L}=\mathscr{C}^{\mathrm{III}}$. 
For the case (iii), if the chain led by $\mathscr{C}_{N}^{\mathrm{I}}$ (defined by the construction rule in the current section) has the structure shown by figure 32 (c) i.e. this chain ends at one side $\left(\mathscr{C}_{i}^{\mathrm{I}}\right)_{b / t}$ of a component belonging to the chain led by $\mathscr{C}^{\mathrm{II}}$, while the opposite side $\left(\mathscr{C}_{i}^{\mathrm{I}}\right)_{t / b}$ is connected to the part containing $\mathscr{C}^{\mathrm{II}}$ via a type-3 line, we redefine the upper block by the chain (defined in section 6.1) $\mathscr{U} \rightarrow \mathscr{U}^{\prime}=\left[\left(\mathscr{C}_{N}^{\mathrm{I}}\right)_{t}-\left(\mathscr{C}_{N}^{\mathrm{I}}\right)_{b}, \ldots,\left(\mathscr{C}_{i}^{\mathrm{I}}\right)_{b}-\left(\mathscr{C}_{i}^{\mathrm{I}}\right)_{t}, \ldots, \mathscr{U}=\mathscr{C}^{\mathrm{II}}\right]$. Contrarily, if the chain led by $\mathscr{C}_{N}^{\mathrm{I}}$ has the form figure $32(\mathrm{~d})$, we redefine the lower block by the chain (defined in section 6.1) $\mathscr{L} \rightarrow \mathscr{L}^{\prime}=\left[\left(\mathscr{C}_{N}^{\mathrm{I}}\right)_{t}-\left(\mathscr{C}_{N}^{\mathrm{I}}\right)_{b}, \ldots,\left(\mathscr{C}_{i}^{\mathrm{I}}\right)_{t}-\left(\mathscr{C}_{i}^{\mathrm{I}}\right)_{b}, \ldots, \mathscr{L}=\mathscr{C}^{\mathrm{III}}\right]$. After finding out the chain led by $\mathscr{C}_{N}^{\mathrm{I}}$ defined in section 6.1 , we remove the starting and internal components of this chain from the reference order $\mathrm{R}_{\mathscr{C}}^{\mathrm{I}} \equiv \mathrm{R}_{\mathscr{C}} \backslash \mathscr{C}^{\mathrm{II}}=\left\{\mathscr{C}_{1}^{\mathrm{I}}, \mathscr{C}_{2}^{\mathrm{I}}, \ldots, \mathscr{C}_{N}^{\mathrm{I}}\right\}$.

Next, we find out the chain that is led by the highest-weight component in the redefined $\mathrm{R}_{\mathscr{C}}^{\mathrm{I}}$ by following the previous step but using the redefined $\mathrm{R}_{\mathscr{C}}^{\mathrm{I}}, \mathscr{U}$ and $\mathscr{L}$. Repeating these steps until the ordered set $\mathrm{R}_{\mathscr{C}}^{\mathrm{I}}$ becomes empty, we get graphs with only two disjoint maximally connected subgraphs: the final upper and lower blocks. Then the graph is finally given by connecting the final upper and lower blocks $\mathscr{U}$ and $\mathscr{L}$ via a type-3 line such that there is no unphysical structure like figure 12 (a), (b). This description of the physical graphs obtained by the construction rule precisely agrees with the construction of physical graphs in section 6 .

Conversely, any physical graph constructed according to the method in section 6 can also be obtained by the construction rule provided in this section. We define reference order $\mathrm{R}_{\mathscr{C}} \equiv\left\{\mathscr{C}_{1}^{\mathrm{I}}, \mathscr{C}_{2}^{\mathrm{I}}, \ldots, \mathscr{C}_{N}^{\mathrm{I}}, \mathscr{C}^{\mathrm{II}}\right\}$. One can always find out a path from the highest-weight component $\mathscr{C}^{\mathrm{II}}$ in $\mathrm{R}_{\mathscr{C}}$ to $\mathscr{C}^{\mathrm{III}}$. This path can be considered as the chain $\left[\mathscr{C}^{\mathrm{II}}, \ldots, \mathscr{C}^{\mathrm{III}}\right]$ which starts at $\mathscr{C}^{\mathrm{II}}$ and ends at $\mathscr{C}$ III. Redefine the ordered set $\mathrm{R}_{\mathscr{C}}$ by deleting the starting and internal components of this chain.

In the same way, we find out the chain led by the highest-weight component of the reference order $\mathrm{R}_{\mathscr{C}}$ that is defined in the previous step and redefine $\mathrm{R}_{\mathscr{C}}$ again. Repeat these steps until $R_{\mathscr{C}}$ becomes an empty set. Then we find that a physical graph constructed in section 6 can be obtained by the rule in this section.

Open Access. This article is distributed under the terms of the Creative Commons Attribution License (CC-BY 4.0), which permits any use, distribution and reproduction in any medium, provided the original author(s) and source are credited.

\section{References}

[1] R.H. Boels and R. Medina, Graviton and gluon scattering from first principles, Phys. Rev. Lett. 118 (2017) 061602 [arXiv:1607.08246] [INSPIRE].

[2] N. Arkani-Hamed, L. Rodina and J. Trnka, Locality and Unitarity of Scattering Amplitudes from Singularities and Gauge Invariance, Phys. Rev. Lett. 120 (2018) 231602 [arXiv: 1612.02797] [INSPIRE].

[3] R.H. Boels and H. Lüo, A minimal approach to the scattering of physical massless bosons, JHEP 05 (2018) 063 [arXiv: 1710.10208] [INSPIRE].

[4] R.H. Boels, Q. Jin and H. Lüo, Efficient integrand reduction for particles with spin, arXiv: 1802.06761 [INSPIRE]. 
[5] C.-H. Fu, Y.-J. Du, R. Huang and B. Feng, Expansion of Einstein-Yang-Mills Amplitude, JHEP 09 (2017) 021 [arXiv: 1702.08158] [INSPIRE].

[6] M. Chiodaroli, M. Günaydin, H. Johansson and R. Roiban, Explicit Formulae for Yang-Mills-Einstein Amplitudes from the Double Copy, JHEP 07 (2017) 002 [arXiv: 1703.00421] [INSPIRE].

[7] F. Cachazo, S. He and E.Y. Yuan, Scattering equations and Kawai-Lewellen-Tye orthogonality, Phys. Rev. D 90 (2014) 065001 [arXiv:1306.6575] [INSPIRE].

[8] F. Cachazo, S. He and E.Y. Yuan, Scattering of Massless Particles in Arbitrary Dimensions, Phys. Rev. Lett. 113 (2014) 171601 [arXiv:1307.2199] [inSPIRE].

[9] F. Cachazo, S. He and E.Y. Yuan, Scattering of Massless Particles: Scalars, Gluons and Gravitons, JHEP 07 (2014) 033 [arXiv:1309.0885] [InSPIRE].

[10] F. Cachazo, S. He and E.Y. Yuan, Scattering Equations and Matrices: From Einstein To Yang-Mills, DBI and NLSM, JHEP 07 (2015) 149 [arXiv:1412.3479] [INSPIRE].

[11] F. Teng and B. Feng, Expanding Einstein-Yang-Mills by Yang-Mills in CHY frame, JHEP 05 (2017) 075 [arXiv:1703.01269] [INSPIRE].

[12] Y.-J. Du and F. Teng, BCJ numerators from reduced Pfaffian, JHEP 04 (2017) 033 [arXiv: 1703.05717] [INSPIRE].

[13] S. Stieberger and T.R. Taylor, New relations for Einstein-Yang-Mills amplitudes, Nucl. Phys. B 913 (2016) 151 [arXiv:1606.09616] [INSPIRE].

[14] D. Nandan, J. Plefka, O. Schlotterer and C. Wen, Einstein-Yang-Mills from pure Yang-Mills amplitudes, JHEP 10 (2016) 070 [arXiv: 1607.05701] [INSPIRE].

[15] L. de la Cruz, A. Kniss and S. Weinzierl, Relations for Einstein-Yang-Mills amplitudes from the CHY representation, Phys. Lett. B 767 (2017) 86 [arXiv:1607.06036] [INSPIRE].

[16] O. Schlotterer, Amplitude relations in heterotic string theory and Einstein-Yang-Mills, JHEP 11 (2016) 074 [arXiv: 1608.00130] [INSPIRE].

[17] Y.-J. Du, B. Feng and F. Teng, Expansion of All Multitrace Tree Level EYM Amplitudes, JHEP 12 (2017) 038 [arXiv: 1708.04514] [INSPIRE].

[18] R. Britto, F. Cachazo and B. Feng, New recursion relations for tree amplitudes of gluons, Nucl. Phys. B 715 (2005) 499 [hep-th/0412308] [INSPIRE].

[19] R. Britto, F. Cachazo, B. Feng and E. Witten, Direct proof of tree-level recursion relation in Yang-Mills theory, Phys. Rev. Lett. 94 (2005) 181602 [hep-th/0501052] [INSPIRE].

[20] L.A. Barreiro and R. Medina, RNS derivation of $N$-point disk amplitudes from the revisited S-matrix approach, Nucl. Phys. B 886 (2014) 870 [arXiv:1310.5942] [INSPIRE].

[21] Y.-J. Du and Y. Zhang, Gauge invariance induced relations and the equivalence between distinct approaches to NLSM amplitudes, JHEP 07 (2018) 177 [arXiv:1803.01701] [INSPIRE].

[22] Y.-J. Du and C.-H. Fu, Explicit BCJ numerators of nonlinear simga model, JHEP 09 (2016) 174 [arXiv: 1606.05846] [INSPIRE].

[23] J.J.M. Carrasco, C.R. Mafra and O. Schlotterer, Abelian Z-theory: NLSM amplitudes and $\alpha^{\prime}$-corrections from the open string, JHEP 06 (2017) 093 [arXiv:1608.02569] [INSPIRE]. 
[24] R. Kleiss and H. Kuijf, Multi-Gluon Cross-sections and Five Jet Production at Hadron Colliders, Nucl. Phys. B 312 (1989) 616 [inSPIRE].

[25] Z. Bern, J.J.M. Carrasco and H. Johansson, New Relations for Gauge-Theory Amplitudes, Phys. Rev. D 78 (2008) 085011 [arXiv:0805.3993] [INSPIRE].

[26] S. Stieberger, Open 83 Closed vs. Pure Open String Disk Amplitudes, arXiv:0907.2211 [INSPIRE].

[27] N.E.J. Bjerrum-Bohr, P.H. Damgaard and P. Vanhove, Minimal Basis for Gauge Theory Amplitudes, Phys. Rev. Lett. 103 (2009) 161602 [arXiv:0907.1425] [INSPIRE].

[28] B. Feng, R. Huang and Y. Jia, Gauge Amplitude Identities by On-shell Recursion Relation in S-matrix Program, Phys. Lett. B 695 (2011) 350 [arXiv: 1004.3417] [INSPIRE].

[29] Y.-X. Chen, Y.-J. Du and B. Feng, A Proof of the Explicit Minimal-basis Expansion of Tree Amplitudes in Gauge Field Theory, JHEP 02 (2011) 112 [arXiv:1101.0009] [INSPIRE].

[30] R. Huang, Y.-J. Du and B. Feng, Understanding the Cancelation of Double Poles in the Pfaffian of CHY-formulism, JHEP 06 (2017) 133 [arXiv: 1702.05840] [INSPIRE].

[31] C.S. Lam, Pfaffian Diagrams for Gluon Tree Amplitudes, Phys. Rev. D 98 (2018) 076002 [arXiv: 1808.07575] [INSPIRE].

[32] G. Chen, H. Johansson, F. Teng and T. Wang, Kinematic algebra for BCJ numerators beyond the $M H V$ sector, to appear.

[33] A. Hodges, A simple formula for gravitational MHV amplitudes, arXiv:1204.1930 [INSPIRE].

[34] Y.-J. Du, F. Teng and Y.-S. Wu, Direct Evaluation of n-point single-trace MHV amplitudes in 4d Einstein-Yang-Mills theory using the CHY Formalism, JHEP 09 (2016) 171 [arXiv: 1608.00883] [INSPIRE].

[35] D. Nguyen, M. Spradlin, A. Volovich and C. Wen, The Tree Formula for MHV Graviton Amplitudes, JHEP 07 (2010) 045 [arXiv:0907.2276] [INSPIRE].

[36] B. Feng and S. He, Graphs, determinants and gravity amplitudes, JHEP 10 (2012) 121 [arXiv: 1207.3220] [INSPIRE].

[37] X. Gao, S. He and Y. Zhang, Labelled tree graphs, Feynman diagrams and disk integrals, JHEP 11 (2017) 144 [arXiv:1708.08701] [INSPIRE].

[38] S. He, G. Yan, C. Zhang and Y. Zhang, Scattering Forms, Worldsheet Forms and Amplitudes from Subspaces, JHEP 08 (2018) 040 [arXiv: 1803.11302] [INSPIRE]. 